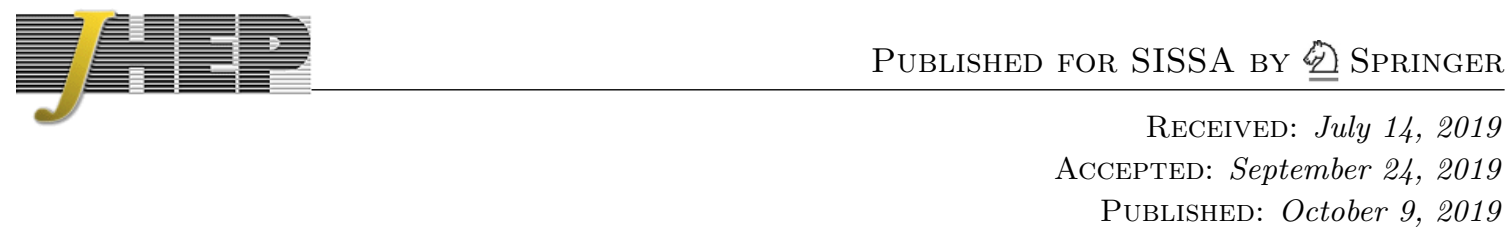

\title{
String field theory as world-sheet UV regulator
}

\author{
Ashoke Sen \\ Harish-Chandra Research Institute, HBNI, \\ Chhatnag Road, Jhusi, Allahabad 211019, India \\ E-mail: sen@hri.res.in
}

AbSTRACT: Even at tree level, the first quantized string theory suffers from apparent short distance singularities associated with collision of vertex operators that prevent us from straightforward numerical computation of various quantities. Examples include string theory S-matrix for generic external momenta and computation of the spectrum of string theory under a marginal deformation of the world-sheet theory. The former requires us to define the S-matrix via analytic continuation or as limits of contour integrals in complexified moduli space, while the latter requires us to use an ultraviolet cut-off at intermediate steps. In contrast, string field theory does not suffer from such divergences. In this paper we show how string field theory can be used to generate an explicit algorithm for computing tree level amplitudes in any string theory that does not suffer from any short distance divergence from integration over the world-sheet variables. We also use string field theory to compute second order mass shift of string states under a marginal deformation without having to use any cut-off at intermediate steps. We carry out the analysis in a broad class of string field theories, thereby making it manifest that the final results are independent of the extra data that go into the formulation of string field theory. We also comment on the generalization of this analysis to higher genus amplitudes.

KEywords: String Field Theory, Bosonic Strings, Superstrings and Heterotic Strings

ARXIV EPRINT: 1902.00263 


\section{Contents}

1 Introduction and summary 1

2 Conventions $\quad 9$

3 Local coordinates and picture changing operators $\quad 14$

$\begin{array}{lll}3.1 \text { Local coordinate system } & 14\end{array}$

$\begin{array}{lll}3.2 & \text { Locations of picture changing operators } & 18\end{array}$

4 Warm up with four point amplitude $\quad 20$

4.1 A concise description of the boundary terms 20

$\begin{array}{lll}4.2 & \text { Four tachyon amplitude } & 22\end{array}$

$\begin{array}{lll}4.3 \text { Comments } & 25\end{array}$

5 Higher point amplitudes $\quad 26$

$6 \quad$ Amplitudes in superstring theory 33

7 Mass shift under marginal deformation $\quad 34$

$\begin{array}{lll}7.1 & \text { Bosonic string theory } & 35\end{array}$

7.2 Heterotic string theory 43

8 Comments of higher genus amplitudes $\quad 48$

A Choice of local coordinates on a five punctured sphere $\quad 51$

B Analysis of $\mathrm{B}_{u}^{\prime \prime}, \mathrm{B}_{s}$ and $\mathrm{B}_{t} \quad 55$

\section{Introduction and summary}

String field theory was originally formulated with the hope of getting non-perturbative results in string theory. With the exception of some non-perturbative classical solutions in open string field theory [1], this hope has not been realized so far. However string field theory can play another useful role - in getting a better description of perturbative string theory. Indeed, superstring field theory has been useful for giving a definition of perturbative string amplitudes free from all divergences other than the ones expected on physical grounds, e.g. the usual infrared divergences in four space-time dimensions. It has also been useful for proving general properties of the perturbative S-matrix e.g. unitarity, analyticity etc. A review of these developments can be found in [2].

The goal of this paper will be to use string field theory to remove some of the inconvenient features of tree level string theory — divergences associated with short distance 
singularities on the world-sheet. We analyze two classes of problems - computation of on-shell amplitudes and studying the effect of marginal deformation on the world-sheet (super-) conformal field theory (CFT) on the spectrum of string theory. Since at tree level, theories with tachyons e.g. bosonic string theory and superstring theory formulated around certain non-supersymmetric backgrounds, also give sensible results, our analysis will also be valid for the corresponding string field theories. For this reason we shall use the phrase 'string field theory' instead of 'superstring field theory', with the understanding that superstring field theory should be considered as a special case of string field theory. We shall see that all the apparent short distance divergences on the world-sheet arise from wrong treatment of $\left(L_{0}+\bar{L}_{0}\right)^{-1}$ where $L_{n}, \bar{L}_{n}$ are the usual Virasoro generators of matter ghost CFT and, for superstring theory, also from wrong treatment of picture changing operators (PCOs) [3].

There are many versions of string field theory - for our analysis we shall work with a broad class of string field theories whose interaction vertices are constructed in such a way that the equality of the S-matrix computed from string field theory and first quantized string theory is manifest. This includes for example the original open bosonic string field theory of Witten [4], its generalization to closed bosonic string field theory in [5-10] and closed and open superstring field theories described e.g. in [11-16], but does not include some versions of string field theory where this equality is not manifest e.g. those described in [17-21]. Although our analysis can be applied to both open and closed string theories, for definiteness we shall focus on closed string theories. For open string theories, the $L_{0}+\bar{L}_{0}$ factors in the subsequent discussion will be replaced by $L_{0}$ and there will be no analog of the projection to $L_{0}=\bar{L}_{0}$ states.

Let us first consider tree level scattering amplitudes in string theory. The usual definition of these amplitudes is in terms of integrals of certain correlation functions of vertex operators in a CFT, but this often suffers from divergences from regions of integration where the locations of the vertex operators come close to each other. A simple example of this is the Koba-Nielsen formula for multi-tachyon amplitudes in bosonic string theory. Usually we avoid this problem by defining these integrals via analytic continuation, but this prevents us from directly evaluating the integrals numerically when an analytic formula is absent. Refs. [22-24] suggest alternative approaches by regarding the integrals as (limits of) integrals in the complexified moduli space - some aspects of this will be discussed later.

In string field theory the tree level S-matrix is evaluated by summing over Feynman diagrams. Part of the contribution to the S-matrix with $N$ external legs comes from the diagram involving an $N$-point interaction vertex to which the external legs connect, without any internal propagator. ${ }^{1}$ This diagram gives the same world-sheet integral as the first quantized string theory, except that the integration is over a restricted region, avoiding regions where two or more vertex operators come close to each other. These missing contributions are given by Feynman diagrams with one or more internal propagators and

\footnotetext{
${ }^{1}$ We shall use the phrase vertex operator to denote a local operator of the CFT on the world-sheet and the phrase interaction vertex to describe a term in the action of string field theory. These two terms should not be confused.
} 
interaction vertices with less number of external legs. Formally the contribution from the latter diagrams also can be represented as the same world-sheet integral as the first quantized string theory, but the integration runs over regions where one or more vertex operators come close, complementing the region associated with the $N$-string interaction vertex. However this correspondence is only formal. To understand the difference between the formal expression and the actual contribution from the Feynman diagram of string field theory, we first note that in Siegel gauge [25] the propagator of the string field takes the form

$$
2 b_{0} \bar{b}_{0}\left(L_{0}+\bar{L}_{0}\right)^{-1} \delta_{L_{0}, \bar{L}_{0}}=\frac{1}{\pi} b_{0} \bar{b}_{0}\left(L_{0}+\bar{L}_{0}\right)^{-1} \int_{0}^{2 \pi} d \theta e^{-i \theta\left(L_{0}-\bar{L}_{0}\right)},
$$

with some additional numerator factors for the Ramond sector states in superstring theories. Here $b_{0}, \bar{b}_{0}$ denote the $b, \bar{b}$ ghost zero modes and $L_{n}, \bar{L}_{n}$ denote the total Virasoro generators. In order to express the contribution from the Feynman diagrams in the usual form of the string amplitude in the first quantized formalism, we need to express (1.1) as

$$
\begin{aligned}
& \frac{1}{\pi} b_{0} \bar{b}_{0} \int_{0}^{\infty} d s e^{-s\left(L_{0}+\bar{L}_{0}\right)} \int_{0}^{2 \pi} d \theta e^{-i \theta\left(L_{0}-\bar{L}_{0}\right)}=-\frac{1}{2 \pi i} b_{0} \bar{b}_{0} \int_{|q| \leq 1} d^{2} q q^{L_{0}-1} \bar{q}^{\bar{L}_{0}-1} \\
& q \equiv e^{-s-i \theta}, \quad d^{2} q \equiv d q \wedge d \bar{q} .
\end{aligned}
$$

We use the convention that $d s \wedge d \theta$ describes positive integration measure. The collection of the variables $(s, \theta)$ for different propagators, together with the integration variables that enter in the definition of the interaction vertices, become the coordinates of the vertex operators on the world-sheet after some change of variables.

Note however that the equality of (1.1) and (1.2) holds only when both sides act on states with positive $L_{0}+\bar{L}_{0}$ eigenvalues. Even tachyon free superstring field theories have off-shell internal states with negative $L_{0}+\bar{L}_{0}$ eigenvalues since they carry Lorentzian space-time momentum. Acting on such states (1.1) is perfectly well-defined, but (1.2) is divergent. It is these divergences that show up as apparent divergences in the integration over the locations of the vertex operators (punctures) in the first quantized formalism.

String field theory suggests different (but equivalent) ways of resolving this problem. The first approach will be to directly use the expressions for amplitudes given as sum over Feynman diagrams without using (1.2). It is possible to formulate string field theory in a way that the sum over intermediate states converges rapidly even though the sum runs over infinite number of states [2]. The second approach will be to rewrite (1.1) by:

$$
\begin{aligned}
& \frac{1}{\pi} b_{0} \bar{b}_{0}\left[\int_{0}^{\Lambda} d s e^{-s\left(L_{0}+\bar{L}_{0}\right)}+\left(L_{0}+\bar{L}_{0}\right)^{-1} e^{-\Lambda\left(L_{0}+\bar{L}_{0}\right)}\right] \int_{0}^{2 \pi} d \theta e^{-i \theta\left(L_{0}-\bar{L}_{0}\right)} \\
= & -\frac{1}{2 \pi i} b_{0} \bar{b}_{0}\left[\int_{e^{-\Lambda} \leq|q| \leq 1} d^{2} q q^{L_{0}-1} \bar{q}^{\bar{L}_{0}-1}-\frac{1}{\bar{L}_{0}} \int_{|q|=e^{-\Lambda}} d q q^{L_{0}-1} \bar{q}^{\bar{L}_{0}}\right],
\end{aligned}
$$

where $\Lambda$ is a large number and in the last term the integration over $q$ runs in the anticlockwise direction. In the second expression in (1.3) we have used the fact that in the second term the integration over $q$ projects to $L_{0}=\bar{L}_{0}$ states, allowing us to replace 
the $L_{0}+\bar{L}_{0}$ factor in the denominator by $2 \bar{L}_{0}$. The total expression is independent of $\Lambda$ and is equal to (1.1) both for positive and negative values of $L_{0}+\bar{L}_{0}$. However the advantage of this representation is that for large $\Lambda$ the contribution from the first term can be represented as the usual string amplitude with integration range over the world-sheet variables having certain excluded domains corresponding to the $|q|<e^{-\Lambda}$ regions, while the second term inside the square bracket receives appreciable contribution only from finite number of states with $L_{0}+\bar{L}_{0} \leq 0 .^{2}$ A third representation of the propagator (1.1) is:

$$
\frac{1}{\pi} b_{0} \bar{b}_{0}\left[\int_{0}^{\Lambda} d s e^{-s\left(L_{0}+\bar{L}_{0}\right)}+\int_{\Lambda}^{\Lambda+i \infty} d s e^{-s\left(L_{0}+\bar{L}_{0}-i \epsilon\right)}\right] \int_{0}^{2 \pi} d \theta e^{-i \theta\left(L_{0}-\bar{L}_{0}\right)},
$$

where $\epsilon$ is a small positive parameter which we eventually take to 0 . This representation of the propagator was used in [23] to get finite results for string amplitudes. This is equivalent to the Feynman $i \epsilon$ prescription, but applied to tree level diagrams. In order to use (1.4) for numerical evaluation of the amplitude, we must work with a finite $\epsilon$ since for $\epsilon=0$ the integrand is oscillatory and not damped. The actual answer is then obtained by evaluating this expression for various values of $\epsilon$ and then extrapolating the result to $\epsilon \rightarrow 0$.

Of course in all these approaches, we have a genuine divergence when an intermediate state has exactly vanishing $L_{0}+\bar{L}_{0}$. This represents the physical poles of the tree level S-matrix, and happens when we choose the external momenta such that an intermediate state goes on-shell.

In the computation of on-shell amplitudes in superstring theory, the apparent divergence arising from intermediate states with negative $\left(L_{0}+\bar{L}_{0}\right)$ eigenvalue can some time be enhanced due to the use of vertex operators of wrong picture number. String field theory gives a definite prescription for the choice of locations of PCOs when two or more vertex operators come close. This in particular guarantees that only -1 and $-3 / 2$ picture states propagate as intermediate states. If we use the wrong picture number, e.g. by converting some of the NS sector vertex operators into zero picture, states in 0 picture propagate in the intermediate state when a pair of zero picture vertex operators approach each other. This sector has states with negative conformal weights leading to apparent divergences which are not present in the correct treatment of string field theory.

In this paper we show that using the representation (1.3) of the propagator (equivalently representation (1.1) following footnote 2) and the fact that the change in the locations of PCOs results in addition of total derivative terms to the integrand, one can arrive at the following prescription for computing (super-)string tree amplitudes that avoids all divergences:

1. Let us suppose that for an $(n+3)$-point amplitude of an arbitrary set of vertex operators in an arbitrary (compactified) string theory, $\mathcal{I}^{(0)}\left(\sigma_{1}, \cdots, \sigma_{n}\right)$ is the integrand

\footnotetext{
${ }^{2}$ As discussed in the last paragraph of section 3.1, for on-shell amplitudes the organization of various terms based on the representation (1.3) can be reinterpreted as the one based on the representation (1.1) by redefining the interactions vertices of string field theory. This can be achieved by absorbing the first term inside the square bracket in (1.3) and the $e^{-\Lambda\left(L_{0}+\bar{L}_{0}\right)}$ factor from the second term into the definition of the interaction vertices, leaving only the propagator factor proportional to $\left(L_{0}+\bar{L}_{0}\right)^{-1}$. In the string field theory literature, this operation is known as 'adding stubs' [26].
} 
obtained by computing the relevant correlation functions of $(n+3)$ vertex operators on the sphere, with three of the vertex operators at fixed positions and the $n$ vertex operators inserted at $\sigma_{1}, \cdots, \sigma_{n}$. Naively the amplitude is given by the integral of the $2 n$-form $\mathcal{I}^{(0)}$ over the whole moduli space parametrized by $\sigma_{i}$ 's, but this integral diverges from the regions where the vertex operators come close, or equivalently, the sphere degenerates.

2. The basic degenerations are single degenerations where the sphere degenerates into a pair of spheres, one carrying $p$ external punctures and an 'internal' puncture and the other carrying $(n+3-p)$ external punctures and an internal puncture, with $p \geq 2$, $(n+3-p) \geq 2$. The two spheres are sewed to each other by cutting out small disks around the internal punctures and gluing the boundaries of the disks. We label all such degenerations by the label $s$ - different values of $s$ correspond to different ways of dividing $(n+3)$ punctures into $p$ punctures and $(n+3-p)$ punctures. Near such a degeneration we can construct from the $\left\{\sigma_{i}\right\}$ 's a new coordinate system, containing a single complex coordinate $u_{s}$ that vanishes at the degeneration and a set of complex coordinates $m_{(s)}$ labelling the moduli of the pair of punctured spheres into which the original sphere degenerates.

3. Multiple degenerations correspond to the region of the moduli space where the original sphere degenerates into several spheres. A degeneration into $(k+1)$ spheres is characterized uniquely by a set of $k$ unordered labels $s_{1}, \cdots, s_{k}$ where each $s_{i}$ corresponds to one of the labels characterizing single degenerations. Near such degenerations we can construct from the $\left\{\sigma_{i}\right\}$ 's a set of complex coordinates $u_{s_{1}}, \cdots, u_{s_{k}}$ that vanish at the degeneration and another set of $n-k$ complex coordinates $m_{\left(s_{1}, \cdots, s_{k}\right)}$, labelling the moduli of the punctured spheres to which the original sphere degenerates.

4. We now denote by $\mathcal{D}_{s}$ an open tubular neighborhood of the subspace $u_{s}=0$ and define $\mathcal{C}^{(0)}$ to be the region of the moduli space that excludes $\mathcal{D}_{s}$ for all $s .^{3}$ Therefore by construction $\mathcal{C}^{(0)}$ excludes all degenerations. We also denote by $\mathcal{C}_{s}^{(1)}$ the intersection $\partial \mathcal{D}_{s} \cap \mathcal{C}^{(0)}$, forming a component of the boundary of $\mathcal{C}^{(0)}$, and by $\mathcal{C}_{s_{1} \cdots s_{k}}^{(k)}$ the codimension $k$ intersection $\mathcal{C}_{s_{1}}^{(1)} \cap \mathcal{C}_{s_{2}}^{(1)} \cap \cdots \cap \mathcal{C}_{s_{k}}^{(1)}$. The orientation of $\mathcal{C}_{s_{1} \cdots s_{k}}^{(k)}$ is fixed by the equation

$$
\partial \mathcal{C}_{s_{1} \cdots s_{k}}^{(k)}=-\sum_{s} \mathcal{C}_{s_{1} \cdots s_{k} s}^{(k+1)}
$$

where the sum over $s$ runs over all labels for which $\mathcal{C}_{s_{1} \cdots s_{k} s}^{(k+1)}$ exists.

\footnotetext{
${ }^{3}$ For example in a local patch we could take $\left|u_{s}\right|<\epsilon$ for some small number $\epsilon$ as the definition of $\mathcal{D}_{s}$. The choice of $u_{s}$ 's is of course not unique, but the final result is independent of this choice. Furthermore the coordinates $\left\{u_{s_{1}}, \cdots, u_{s_{k}}\right\}$ may need to be defined differently in different local patches of the space spanned by $m_{\left(s_{1}, \cdots, s_{k}\right)}$. In this case on the overlap one needs to choose appropriate interpolation in the definition of $\mathcal{D}_{s}$ between the two patches. An explicit example elaborating the choice of the coordinates $\left\{u_{s}\right\},\left\{m_{\left(s_{1}, \cdots, s_{k}\right)}\right\}$ and the definition of $\mathcal{D}_{s}$ for a five punctured sphere has been described in appendix A.
} 
5. Then the full amplitude (for generic external momenta) is given by

$$
I=\sum_{k=0}^{n} \sum_{\substack{\left\{s_{1}, \cdots, s_{k}\right\} \\ s_{1}<s_{2}<\cdots<s_{k}}} \int_{\mathcal{C}_{s_{1} \cdots s_{k}}^{(k)}} \mathcal{I}_{s_{1} \cdots s_{k}}^{(k)},
$$

where the $(2 n-k)$ forms $\mathcal{I}_{s_{1} \cdots s_{k}}^{(k)}$, defined in a neighborhood of $\mathcal{C}_{s_{1} \cdots s_{k}}^{(k)}$, are obtained by solving the equations

$$
d \mathcal{I}_{s_{1} \cdots s_{k}}^{(k)}=\mathcal{I}_{s_{1} \cdots s_{k-1}}^{(k-1)}-\mathcal{I}_{s_{1} \cdots s_{k-2} s_{k}}^{(k-1)}+\cdots+(-1)^{k-1} \mathcal{I}_{s_{2} \cdots s_{k}}^{(k-1)}
$$

Beginning with the known expression for $\mathcal{I}^{(0)}$, we could solve for $\mathcal{I}_{s_{1} \cdots s_{k}}^{(k)}$ iteratively in $k$ by expanding both sides of (1.7) in a power series in $u_{s_{1}}, \cdots, u_{s_{k}}$ and their complex conjugates and matching the coefficients.

6. The solution to (1.7) is not unique but the final result is not affected by this ambiguity, as long as we work with generic external momenta so that the exponents in the power series expansion in $u_{s}, \bar{u}_{s}$ are not integers. Similarly the choice of $\mathcal{C}_{s}^{(1)}$, s and therefore of the $\mathcal{C}_{s_{1} \cdots s_{k}}^{(k)}$ 's depend on the choice of the tubular neighborhoods $\mathcal{D}_{s}$ which are not unique. However the expression (1.6) can be shown to be invariant under deformations of the $\mathcal{C}_{s}^{(1)}$, s.

Note that for $k=0$ term in (1.6), the integrand is the same as the original integrand but the integration runs over the region $\mathcal{C}^{(0)}$ that avoids all degenerations. The missing regions are compensated for by the remaining integrals involving $k \geq 1$ terms. Also note that the final formula for the amplitude encoded in (1.6) and (1.7) does not require any detailed knowledge of string field theory even though we use string field theory to arrive at these formulæ.

We can regard $\int_{\mathcal{C}_{s_{1} \cdots s_{k}}^{(k)}} \mathcal{I}_{s_{1} \cdots s_{k}}^{(k)}$ as the result of using the second term in (1.3) - or equivalently (1.4) - for the propagators labelled by $s_{1}, \cdots, s_{k}$ if we identify the variable $u_{s}$ with the variable $q$ for the $s$-th propagator and the tubular neighborhood $\mathcal{D}_{s}$ as the region $\left|u_{s}\right|<e^{-\Lambda}$. As we shall see, this choice of $\left\{u_{s}\right\}$ and $\left\{\mathcal{D}_{s}\right\}$ is not strictly necessary. Nevertheless this makes the connection to the $i \epsilon$ prescription of [23] clear: $\int_{\mathcal{C}_{s_{1} \cdots s_{k}}^{(k)}} \mathcal{I}_{s_{1} \cdots s_{k}}^{(k)}$ represents part of the contribution where the integral over $\ln \left|u_{s}\right|$ runs parallel to the imaginary axis for $s=s_{1}, \cdots, s_{k}$. We note however that the prescription given in (1.6) works equally well for complex external momenta, while the $i \epsilon$ prescription will typically require different choices of the contour of integration in the complexified moduli space for different complex external momenta to ensure convergence.

The procedure described above works even if the original integrand $\mathcal{I}^{(0)}$ is computed with wrong choice of PCO locations, e.g. by taking the PCO locations to coincide with some of the vertex operators. This adds a total derivative to $\mathcal{I}^{(0)}$ and could introduce additional divergences when the vertex operators collide. However the addition of such terms to $\mathcal{I}^{(0)}$ also requires us to add corrections to $\mathcal{I}_{s}^{(1)}$ when we solve $(1.7)$, and these cancel in (1.6). Therefore while implementing this procedure, one does not need to be careful about the choice of PCO locations. 
As already mentioned, during the analysis described above we do not assume any particular choice of string background or any specific form of the external states. Our analysis holds for generic external states in a generic (compactified) string theory.

The second application of classical string field theory that we discuss is in the study of marginal deformations of the world-sheet CFT describing the background space-time. String field theory is formulated around some specific classical solution, corresponding to a CFT of matter and ghost fields on the world-sheet. If this CFT has marginal deformations, then one can in principle compute the spectrum and correlations functions of all the operators in the deformed theory using conformal perturbation theory. This in turn will determine the spectrum of physical states and the S-matrix of string theory formulated in the deformed background, in terms of the spectrum and correlation functions of the original CFT. In practice this requires carefully regulating the ultraviolet divergences on the world-sheet since the marginal operator needs to be integrated on the world-sheet together with other vertex operators and we have to regulate the divergences when the locations of the operators come close to each other. In particular at higher order in perturbation theory when there are several insertions of the marginal operator we need to carefully remove all the divergences, while making sure not to remove the finite pieces.

Now given the deformed CFT, one can formulate a string field theory around this deformed CFT. It is known that this new string field theory is related to the string field theory formulated around the original CFT by a field redefinition that includes a shift [27-29]. This means that the original string field theory has a classical solution that describes string field theory around the deformed background. ${ }^{4}$ Furthermore the spectrum and S-matrix of the string field theory around the deformed CFT can be computed by expanding the original string field theory action around the classical solution describing the deformed background. Since string field theory has no divergences, this procedure also does not suffer from any divergences. In other words string field theory automatically provides an ultraviolet regulator for the world-sheet theory. A fully systematic procedure for constructing the classical solution in string field theory to any given order in the expansion in terms of the deformation parameters, and computing the spectrum and S-matrix of string theory in the deformed background, can be found in $[2,35]$. Its application to a particular class of examples has also been described in [36] (see also [37, 38] for construction of the solution). The apparent ultraviolet divergences in the conformal perturbation theory can be traced either to wrong use of Schwinger parametrization as in (1.2), or applying $\left(L_{0}+\bar{L}_{0}\right)^{-1}$ on states that have zero $\left(L_{0}+\bar{L}_{0}\right)$ eigenvalue. The first case is dealt with by using (1.1) or (1.3), while the second case requires a more elaborate treatment as explained in sections 3.3 and 4.1 of [35].

We apply this method to the special case of string compactification on a circle, and consider the marginal deformation to be the one that changes the radius of the circle. The mass of a state carrying momentum $n / R$ along the circle gets shifted under this deformation. We find that both for the bosonic and the heterotic string theory the mass ${ }^{2}$ shift to second order in the deformation parameter $\mu$ is given by

$$
\Delta m^{2}=-n^{2} R^{-2}\left(\mu-\frac{\mu^{2}}{2}\right) .
$$

\footnotetext{
${ }^{4}$ For open string field theory, such classical solutions have been constructed analytically [30-34].
} 
Since the leading order expression for $m^{2}$ has an additive term given by $n^{2} R^{-2}$, (1.8) corresponds to a scaling of $R^{-2}$ by $\left(1-\mu+\mu^{2} / 2\right)$. This is consistent since the marginal deformation is expected to change the radius. If $\Delta m^{2}$ had not been proportional to $n^{2}$ then this would not be possible. Indeed at the intermediate stages of the calculation there are other terms proportional to $n^{4} R^{-4}$ and $n^{0} R^{0}$, but they cancel at the end. Nowhere at any stage of the calculation we need an ultraviolet regulator on the world-sheet. Furthermore the method we use is completely general and can be used to compute the shift in the spectrum under a general marginal deformation where the answer may not be a priori known, e.g. shift in the masses of heavy string states under a blowup of the orbifold singularity.

Another possible application of string field theory is in the study of Ramond-Ramond background [39]. Once a string field theory is formulated around a given world-sheet CFT, one may have a family of consistent classical solutions that involve switching on RamondRamond background. In this case one can use the same procedure used for marginal deformations to systematically construct the classical solution in string field theory to any order in deformation parameter, and compute the spectrum and S-matrix of the theory around the deformed background [39]. As in the case of marginal deformations, this procedure never requires an ultraviolet regulator on the world-sheet if we apply the formalism of string field theory systematically.

As is well known, formulation of string field theory requires us to specify certain data - the choice of local coordinates at the punctures of the Riemann surface and locations of the PCOs. The results we have quoted are independent of the data. Indeed, one of the goals in our analysis will be to manipulate the various expressions in such a way that even though at the intermediate stages of the calculation the result depends on these additional data (which we leave unspecified), this dependence cancels at the end. This is manifest in our expressions (1.6) and (1.7). One finds for example that different choices of local coordinate system at the punctures lead to different choices of the subspaces $\mathcal{C}_{s}^{(1)}$ (and hence also $\mathcal{C}_{s_{1} \cdots s_{k}}^{(k)}$ ), but it can be shown that (1.6) is invariant under deformations of the $\mathcal{C}_{s}^{(1)}$ 's. ${ }^{5}$ Similarly different choices of PCO locations can be shown to induce an additive total derivative term in the expression for $\mathcal{I}^{(0)}$, but (1.6) can be shown to remain unchanged under addition of such a term. In essence, such an additive term in $\mathcal{I}^{(0)}$ forces us to also add certain terms in $\mathcal{I}_{s}^{(1)}$ in order to satisfy (1.7). These extra terms cancel the effect of additive term in $\mathcal{I}^{(0)}$ when we evaluate (1.6).

Similarly we see that (1.8) does not depend on the choice of local coordinate systems or PCO locations. At intermediate stages of the analysis various expressions we get do depend on the additional data, but this dependence cancels at the end. We must note however that the total independence of the result (1.8) of the additional data is accidental since different choice of local coordinates and PCO locations lead to string field theories that are related by field redefinition and we expect that under such a field redefinition the

\footnotetext{
${ }^{5}$ For example if we use hyperbolic metric to introduce the local coordinate system as in [40-42] then the subspaces $\mathcal{C}_{s}^{(1)}$ 's will be obtained by setting to some small number $\ell_{0}$ the length of the closed geodesic that shrinks to a point at the $s$-th degeneration of the punctured sphere. Similar regularization was used in [43] for computing tree level string amplitudes with large number of external states, but only the $\int_{\mathcal{C}^{(0)}} \mathcal{I}^{(0)}$ contribution was analyzed in [43].
} 
deformation parameter $\mu$ - which is the component of a field — will also get redefined. Therefore at higher order in the expansion in powers of $\mu$, we do expect the result to depend on the additional data involved in the construction of string field theory, but this dependence should be removable by a redefinition of $\mu$.

The analysis leading to (1.8) is close to the spirit of the analysis in [39] in that the latter paper computed the shift in mass under deformation involving RR background to second order in the deformation parameter $\mu$. There is however one important technical difference between the two analysis. In the case of RR background, the leading contribution to the mass shift appears at second order in $\mu$. Therefore for computing the mass shift, the vertex operators of the states whose mass shift is being calculated could be taken to be dimension zero primaries. For the deformation we consider in this paper, the first correction to the mass already appears at order $\mu$, and therefore to compute the mass at second order in $\mu$ we need to use vertex operators whose dimensions differ from zero by order $\mu$. This introduces non-trivial dependence on the choice of local coordinate system at intermediate steps of the calculation even though the dependence cancels at the end. This difference is similar to the difference between the calculation of one loop mass renormalization and two loop mass renormalization. The former requires computing the torus two point function of a pair of vertex operators that satisfy tree level on-shell condition. However the latter requires use of vertex operators that take into account one loop mass renormalization, and therefore do not satisfy the tree level on-shell condition.

The rest of the paper is organized as follows. In section 2 we review some conventions we use to compute correlation functions in the world-sheet CFT and briefly review some aspects of string field theory that we need for our analysis. In section 3 we discuss how choice of local coordinate system and PCO locations affect the results for Feynman diagrams in string field theory. In section 4 we make use of the representation (1.3) of the propagator to give a manifestly finite expression for the four tachyon amplitude in bosonic string theory. In section 5 we use (1.3) to give a general algorithm for getting manifestly finite expressions for general amplitudes in bosonic string theory involving arbitrary number of external states carrying arbitrary quantum numbers. Our analysis in this section leads to eqs. (1.6), (1.7). In section 6 we use superstring field theory to arrive at the same formulæ (1.6), (1.7), giving manifestly finite expressions for the superstring tree amplitudes. In particular we show that (1.6), (1.7) give the correct amplitude even if $\mathcal{I}^{(0)}$ is computed using wrong choice of PCO locations. In section 7 we apply string field theory to study the effect of marginal deformations in bosonic and heterotic string theory and arrive at (1.8) in both theories. We conclude in section 8 with some comments on higher genus amplitudes. Appendix A contains examples of the choice of the coordinate

$\left\{u_{s}\right\},\left\{m_{\left(s_{1} \cdots s_{k}\right)}\right\}$ for a five punctured sphere. Appendix B contains some technical results needed to complete the analysis in section 7.2.

\section{Conventions}

In this section we shall briefly review some aspects of the world-sheet theory and the string field theory that we shall need for our analysis. More details can be found in [2]. 
We begin by describing the normalization conventions for the vacuum of the world-sheet (super-)conformal field theory of the matter ghost system. For bosonic string theory the ghost system has $b, c, \bar{b}$ and $\bar{c}$ ghosts with the usual mode expansion. We normalize the $\mathrm{SL}(2, \mathrm{C})$ invariant vacuum of the bosonic string as

$$
\left\langle 0\left|c_{-1} \bar{c}_{-1} c_{0} \bar{c}_{0} c_{1} \bar{c}_{1}\right| 0\right\rangle=-1,
$$

up to a factor given by the overall volume of space-time which eventually generates the momentum conserving delta function in a correlator. We shall not write this factor explicitly. For heterotic string theory, besides the $b, c, \bar{b}, \bar{c}$ ghost fields we also have the $\beta, \gamma$ ghosts, related to the $\xi, \eta, \phi$ system via the relations

$$
\beta=\partial \xi e^{-\phi}, \quad \gamma=\eta e^{\phi} .
$$

We choose the normalization of the vacuum of the heterotic string such that

$$
\left\langle 0\left|c_{-1} \bar{c}_{-1} c_{0} \bar{c}_{0} c_{1} \bar{c}_{1} e^{-2 \phi(z)}\right| 0\right\rangle=1 .
$$

For type II string theories we also have anti-holomorphic $\bar{\beta}, \bar{\gamma}$ system and the normalization of the vacuum will be chosen as

$$
\left\langle 0\left|c_{-1} \bar{c}_{-1} c_{0} \bar{c}_{0} c_{1} \bar{c}_{1} e^{-2 \phi(z)} e^{-2 \bar{\phi}(\bar{z})}\right| 0\right\rangle=-1 .
$$

We shall denote by $X^{\mu}$ the world-sheet fields corresponding to non-compact space time coordinates, and, for the analysis in section 7 , by $Y$ the world-sheet field corresponding to a compact space direction of radius $R$. We also denote by $\psi^{\mu}$ and $\chi$ their holomorphic superpartners on the world-sheet - in type II theories we also have anti-holomorphic fields $\bar{\psi}^{\mu}$ and $\bar{\chi}$. Their operator product expansions have the form:

$$
\begin{aligned}
\partial X^{\mu}(z) \partial X^{\nu}(w) & =-\frac{\eta^{\mu \nu}}{2(z-w)^{2}}+\cdots, & \psi^{\mu}(z) \psi^{\nu}(w) & =-\frac{\eta^{\mu \nu}}{2(z-w)}+\cdots \\
\partial Y(z) \partial Y(w) & =-\frac{1}{2(z-w)^{2}}+\cdots, & \chi(z) \chi(w) & =-\frac{1}{2(z-w)}+\cdots \\
\bar{\partial} X^{\mu}(\bar{z}) \bar{\partial} X^{\nu}(\bar{w}) & =-\frac{\eta^{\mu \nu}}{2(\bar{z}-\bar{w})^{2}}+\cdots, & \bar{\psi}(\bar{z}) \bar{\psi}^{\nu}(\bar{w}) & =-\frac{\eta^{\mu \nu}}{2(\bar{z}-\bar{w})}+\cdots \\
\bar{\partial} Y(\bar{z}) \bar{\partial} Y(\bar{w}) & =-\frac{1}{2(\bar{z}-\bar{w})^{2}}+\cdots, & \bar{\chi}(\bar{z}) \bar{\chi}(\bar{w}) & =-\frac{1}{2(\bar{z}-\bar{w})}+\cdots
\end{aligned}
$$

where $\cdots$ denote less singular terms whose knowledge will not be needed for our analysis. There may also be additional component of the matter CFT describing other compact directions that will not be relevant for our analysis. The operator product expansion of the ghost fields take the form

$$
\begin{aligned}
c(z) b(w) & =(z-w)^{-1}+\cdots, & \xi(z) \eta(w) & =(z-w)^{-1}+\cdots, \\
\bar{c}(\bar{z}) \bar{b}(\bar{w}) & =(\bar{z}-\bar{w})^{-1}+\cdots, & \bar{\xi}(\bar{z}) \bar{\eta}(\bar{w}) & =(\bar{z}-\bar{w})^{-1}+\cdots, \\
e^{q_{1} \phi(z)} e^{q_{2} \phi(w)} & =(z-w)^{-q_{1} q_{2}} e^{\left(q_{1}+q_{2}\right) \phi(w)}+\cdots, & \partial \phi(z) \partial \phi(w) & =-\frac{1}{(z-w)^{2}}+\cdots, \\
e^{q_{1} \bar{\phi}(\bar{z})} e^{q_{2} \bar{\phi}(\bar{w})} & =(\bar{z}-\bar{w})^{-q_{1} q_{2}} e^{\left(q_{1}+q_{2}\right) \bar{\phi}(\bar{w})}+\cdots, & \bar{\partial} \bar{\phi}(\bar{z}) \bar{\partial} \bar{\phi}(\bar{w}) & =-\frac{1}{(\bar{z}-\bar{w})^{2}}+\cdots,
\end{aligned}
$$

where $\cdots$ denote less singular terms. 
In the heterotic string theory we have holomorphic PCO given by

$$
\mathcal{X}(z)=\left\{Q_{B}, \xi(z)\right\}=c \partial \xi+e^{\phi} T_{F}-\frac{1}{4} \partial \eta e^{2 \phi} b-\frac{1}{4} \partial\left(\eta e^{2 \phi} b\right),
$$

where $Q_{B}$ is the BRST charge:

$$
\begin{aligned}
Q_{B} & =\oint d z \jmath_{B}(z)+\oint d \bar{z} \bar{\jmath}_{B}(\bar{z}), \\
\bar{\jmath}_{B}(\bar{z}) & =\bar{c}(\bar{z}) \bar{T}_{m}(\bar{z})+\bar{b}(\bar{z}) \bar{c}(\bar{z}) \bar{\partial} \bar{c}(\bar{z}), \\
\jmath_{B}(z) & =c(z)\left(T_{m}(z)+T_{\beta, \gamma}(z)\right)+\gamma(z) T_{F}(z)+b(z) c(z) \partial c(z)-\frac{1}{4} \gamma(z)^{2} b(z) .
\end{aligned}
$$

$T_{m}, \bar{T}_{m}$ denote components of the matter stress tensor and $T_{\beta, \gamma}$ is the stress tensor of the $\beta, \gamma$ system. $\oint_{z}$ includes the $1 / 2 \pi i$ factor for holomorphic integral and $-1 / 2 \pi i$ factor for the anti-holomorphic integral. $T_{F}$ is the super-stress tensor of the matter SCFT, given by

$$
T_{F}(z)=-\psi_{\mu} \partial X^{\mu}-\chi \partial Y+\left(T_{F}\right)_{\text {int }} .
$$

Here $\left(T_{F}\right)_{\text {int }}$ denotes the contribution from the additional compact target space directions other than the $Y$ - $\chi$ system. In the bosonic string theory the contribution from the $\beta, \gamma$ system will be absent, while in type II theory there will be additional contribution involving $\bar{\beta}, \bar{\gamma}$. In type II theory we also have the anti-holomorphic PCO, obtained by replacing the holomorphic fields by anti-holomorphic fields in (2.7).

In the bosonic string theory the physical unintegrated vertex operators take the form $c \bar{c} V$ where $V$ is a dimension $(1,1)$ primary in the matter CFT. From this one can construct the integrated vertex operator:

$$
\left(-\oint_{z} d w b(w)\right)\left(-\oint_{z} d \bar{w} \bar{b}(\bar{w})\right) c(z) \bar{c}(\bar{z}) V(z, \bar{z})=-V(z, \bar{z}),
$$

where $\oint_{z}$ denotes a contour around $z$.

In the heterotic string theory the unintegrated -1 picture NS sector vertex operator takes the form

$$
c \bar{c} e^{-\phi} V
$$

where $V$ is a dimension $(1,1 / 2)$ superconformal primary in the matter SCFT. The unintegrated zero picture vertex operator takes the form

$$
\lim _{w \rightarrow z} \mathcal{X}(w) c \bar{c} e^{-\phi} V(z, \bar{z})=c \bar{c} W(z, \bar{z})-\frac{1}{4} \eta \bar{c} e^{\phi} V(z, \bar{z}),
$$

where $W$ is a dimension $(1,1)$ matter sector vertex operator defined via

$$
W(z, \bar{z})=-\lim _{w \rightarrow z}(w-z) T_{F}(w) V(z, \bar{z}) .
$$

The integrated -1 picture vertex operator takes the form

$$
\left(-\oint_{z} d w b(w)\right)\left(-\oint_{z} d \bar{w} \bar{b}(\bar{w})\right) c \bar{c} e^{-\phi} V(z, \bar{z})=-e^{-\phi} V(z, \bar{z}),
$$


and the integrated 0 picture vertex operator is given by

$$
\left(-\oint_{z} d w b(w)\right)\left(-\oint_{z} d \bar{w} \bar{b}(\bar{w})\right)\left[c \bar{c} W(z, \bar{z})-\frac{1}{4} \eta \bar{c} e^{\phi} V(z, \bar{z})\right]=-W(z, \bar{z}) .
$$

In type II string theory we can similarly define integrated and unintegrated NSNS sector vertex operators carrying picture numbers $(-1,-1),(0,-1),(-1,0)$ and $(0,0)$. We shall not write down the explicit form of the Ramond sector vertex operators since they will not be needed for our analysis.

Tree level $(n+3)$-point amplitude of vertex operators $c \bar{c} V_{i}$ for $1 \leq i \leq(n+3)$ in bosonic string theory is given by converting $n$ of them to integrated vertex operators and integrating the resulting correlation function over the locations of the integrated vertex operators:

$$
A=\left(-\frac{1}{2 \pi i}\right)^{n} \int \prod_{i=1}^{n} d \sigma_{i} \wedge d \bar{\sigma}_{i}\left\langle c \bar{c} V_{n+1}\left(y_{n+1}\right) c \bar{c} V_{n+2}\left(y_{n+2}\right) c \bar{c} V_{n+3}\left(y_{n+3}\right) \prod_{i=1}^{n}\left(-V_{i}\left(\sigma_{i}\right)\right)\right\rangle .
$$

Here $y_{n+1}, y_{n+2}$ and $y_{n+3}$ represent arbitrary points in the complex plane. The $(-1 / 2 \pi i)^{n}$ factor is a normalization factor that appears in the definition of the interaction vertices of string field theory and is related to the $-1 / 2 \pi i$ factor in (1.2). $A$ given in (2.15) can be regarded as a contribution to the $T$-matrix, related to the $S$-matrix by $S=1+i T$.

The results for the tree level $(n+3)$ point function of NS sector states in the heterotic string theory and NSNS sector states in type II string theories are similar, except that the vertex operators are taken in the -1 picture and the amplitude has insertion of $(n+1)$ of PCOs in the heterotic theory and $(n+1)$ holomorphic PCO's and $(n+1)$ pair of antiholomorphic PCO's in type II theory. The PCO locations are arbitrary when the vertex operators are well separated from each other but need to satisfy certain relations when two or more vertex operators approach each other. These rules are induced from superstring field theory and essentially tell us that when $n$ vertex operators approach each other, we must also have $(n-1)$ PCOs approaching them so that the picture numbers of all the operators add up to -1 . If there are $\mathrm{R}$ sector vertex operators present, then they are taken in the $-1 / 2$ picture. The number of PCO's need to be adjusted so that the total picture number of all vertex operators and PCOs add up to -2 in the heterotic string theory and $(-2,-2)$ in the type II string theory.

We shall now briefly review some aspects of (super-)string field theory. In bosonic string theory we denote by $\mathcal{H}$ the Hilbert space of matter-ghost CFT satisfying the conditions:

$$
|\phi\rangle \in \mathcal{H} \quad \text { if } b_{0}^{-}|\phi\rangle=0, L_{0}^{-}|\phi\rangle=0, \quad b_{0}^{ \pm} \equiv b_{0} \pm \bar{b}_{0}, \quad L_{0}^{ \pm} \equiv L_{0} \pm \bar{L}_{0}, \quad c_{0}^{ \pm} \equiv \frac{1}{2}\left(c_{0} \pm \bar{c}_{0}\right) .
$$

For NS sector of heterotic string theory and NSNS sector of type II string theory we have similar constraints except that we also require the states in $\mathcal{H}$ to carry picture number -1 in heterotic string theory and picture number $(-1,-1)$ in type II string theory. In all the theories the string field $|\Psi\rangle$ is an arbitrary element of $\mathcal{H}$. We shall denote by $\Psi$ the corresponding vertex operator in the CFT. 
The classical action of bosonic string field theory and the NS sector fields in superstring field theory takes the form: ${ }^{6}$

$$
S=\frac{1}{2}\left\langle\Psi\left|c_{0}^{-} Q_{B}\right| \Psi\right\rangle+\sum_{N=3}^{\infty} \frac{1}{N !}\left\{\Psi^{N}\right\},
$$

where for $\left|A_{i}\right\rangle \in \mathcal{H},\left\{A_{1} \cdots A_{n}\right\}$ is a multilinear function of the $A_{i}$ 's obtained by first constructing the sphere correlation functions of certain ghost operators and PCOs and the vertex operators $A_{i}$ in specified coordinate system, and then integrating the result over an appropriate subspace of the moduli space that excludes all the singular regions where two or more vertex operators come close. The precise choice of these subspaces, or the coordinate system in which the vertex operators are inserted, or the PCO locations are not fixed completely but are subject to stringent constraints, and different choices lead to different string field theories which are related by field redefinition. Note that in this definition we do not require the $A_{i}$ 's to be BRST invariant.

We also define $\left[A_{2} \cdots A_{n}\right] \in \mathcal{H}$ such that

$$
\left\langle A_{1}\left|c_{0}^{-}\right|\left[A_{2} \cdots A_{N}\right]\right\rangle=\left\{A_{1} \cdots A_{n}\right\} \quad \text { for }\left|A_{i}\right\rangle \in \mathcal{H} .
$$

In terms of this, the equation of motion of the string field $|\Psi\rangle$, derived from the action (2.17), takes the form:

$$
Q_{B}|\Psi\rangle+\sum_{N=2}^{\infty} \frac{1}{N !}\left[\Psi^{N}\right]=0 .
$$

Inclusion of the Ramond (R) sector states requires additional structure. In heterotic string theory we introduce a pair of string fields $\Psi_{R}$ and $\widetilde{\Psi}_{R}$ belonging to $-1 / 2$ and $-3 / 2$ picture number sectors satisfying (2.16). In superstring theory $\Psi_{R}$ will include fields in the $(-1,-1 / 2),(-1 / 2,-1)$ and $(-1 / 2,-1 / 2)$ sectors while $\widetilde{\Psi}_{R}$ will include fields in the $(-1,-3 / 2),(-3 / 2,-1)$ and $(-3 / 2,-3 / 2)$ sectors. The kinetic term takes the form

$$
-\frac{1}{2}\left\langle\widetilde{\Psi}_{R}\left|c_{0}^{-} Q_{B} \mathcal{G}\right| \widetilde{\Psi}_{R}\right\rangle+\left\langle\widetilde{\Psi}_{R}\left|c_{0}^{-} Q_{B}\right| \Psi_{R}\right\rangle .
$$

In heterotic string theory $\mathcal{G}$ is the zero mode of the PCO. In type II string theory $\mathcal{G}$ is the zero mode of the holomorphic (anti-holomorphic) PCOs in the NSR (RNS) sectors and product of zero modes of holomorphic and anti-holomorphic PCOs in the RR sector. The interaction term involving the NS and R sector fields have the form

$$
\sum_{N=3}^{\infty} \frac{1}{N !}\left\{\left(\Psi+\Psi_{R}\right)^{N}\right\} .
$$

In particular the field $\widetilde{\Psi}_{R}$ does not enter the interaction term. Therefore the Feynman rules only require the $\Psi-\Psi$ propagator. In Siegel gauge this has the form similar to (1.1) except for an extra factor of $\mathcal{G}$ :

$$
2 b_{0} \bar{b}_{0}\left(L_{0}+\bar{L}_{0}\right)^{-1} \mathcal{G} \delta_{L_{0}, \bar{L}_{0}} .
$$

\footnotetext{
${ }^{6}$ For notational simplicity we have dropped the string coupling constant $g_{s}$ from this expression. It appears in the action via an overall multiplicative factor $g_{s}^{-2}$.
} 
The relevant equation of motion in the Ramond sector, obtained by taking a linear combination of the equations of motion of $\Psi_{R}$ and $\widetilde{\Psi}_{R}$, is given by

$$
Q_{B}\left|\Psi_{R}\right\rangle+\sum_{N=2}^{\infty} \frac{1}{N !} \mathcal{G}\left[\left(\Psi+\Psi_{R}\right)^{N}\right]=0 .
$$

The other linear combination satisfies free field equations of motion and decouples from the theory. Of course we must also replace $\Psi$ by $\Psi+\Psi_{R}$ in the second term in (2.19).

\section{Local coordinates and picture changing operators}

As mentioned earlier, for the construction of a string field theory action, we need to define the interaction vertices involving off-shell string fields. For $n$-point interaction vertex this requires identifying certain subspaces of the moduli space of a sphere with $n$ punctures, and a choice of local coordinates at the punctures, satisfying certain consistency conditions. For superstring theory we also need to specify choice of the locations of the picture changing operators. In this section we shall describe some general criteria for choosing the local coordinate system at the punctures of a three punctured sphere involved in the definition of 3-string interaction vertex and the corresponding choice of a subspace of the moduli space of a sphere with 4 punctures, involved in the definition of the 4-point interaction vertex. We shall also discuss the general criteria for the choice of locations of the PCOs. However we shall refrain from committing ourselves to any particular choice of local coordinates or PCO locations since one of our goals will be to demonstrate that the final result is independent of these choices.

\subsection{Local coordinate system}

On a sphere with three punctures, we can use $\mathrm{SL}(2, \mathrm{C})$ transformation to choose the locations of the punctures to be at

$$
z_{1}=0, \quad z_{2}=\infty, \quad z_{3}=1
$$

We shall choose the local coordinates $w_{i}$ at these punctures to be related to the global coordinate $z$ by

$$
w_{1}=\lambda h_{1}(z), \quad w_{2}=\lambda h_{2}(z), \quad w_{3}=\lambda h_{3}(z)
$$

up to arbitrary phases. $h_{i}(z)$ satisfies $h_{i}\left(z_{i}\right)=0$ for $1 \leq i \leq 3$, so that $w_{i}$ vanishes at $z=z_{i} . \quad \lambda$ is an arbitrary positive real number that could have been included in the definitions of $h_{i}(z)$, but we have displayed it explicitly since at the end we shall try to simplify our analysis by taking the large $\lambda$ limit. $h_{i}(z)$ is an analytic map between an open neighborhood around $z=z_{i}$ and the unit disk $\left|w_{i}\right|<1$, but may have singularities outside this domain. We require the images of the $\left|w_{i}\right| \leq 1$ regions in the $z$-plane to be non-overlapping - this can be achieved by taking $\lambda$ sufficiently large. Furthermore, up to overall phases, this choice of local coordinates should be invariant under permutation of 
the punctures. For example, since the transformation $z \rightarrow 1 / z$ exchanges the punctures 1 and 2 and leaves $z_{3}$ invariant, we must have

$$
h_{1}(1 / z)=h_{2}(z), \quad h_{3}(1 / z)=h_{3}(z),
$$

up to phases. Similarly we should have

$$
h_{1}(1-z)=h_{3}(z), \quad h_{2}(1-z)=h_{2}(z),
$$

and

$$
h_{1}(z /(z-1))=h_{1}(z), \quad h_{2}(z /(z-1))=h_{3}(z),
$$

up to phases. ${ }^{7}$

Let us now consider another sphere with three punctures, carrying global coordinate $z^{\prime}$ and the punctures situated at

$$
z_{1}^{\prime}=0, \quad z_{2}^{\prime}=\infty, \quad z_{3}^{\prime}=1,
$$

carrying local coordinates

$$
w_{1}^{\prime}=\lambda h_{1}\left(z^{\prime}\right), \quad w_{2}^{\prime}=\lambda h_{2}\left(z^{\prime}\right), \quad w_{3}^{\prime}=\lambda h_{3}\left(z^{\prime}\right),
$$

around the three punctures. We can construct a two parameter family of spheres with four punctures by sewing the two spheres at their third punctures via the relation

$$
w_{3} w_{3}^{\prime}=e^{-s-i \theta} \equiv q, \quad 0 \leq s<\infty, \quad 0 \leq \theta<2 \pi .
$$

This family of four punctured spheres is what we shall obtain from the s-channel Feynman diagram of string field theory. Using (3.2) and (3.7) we get the relation between the global coordinates $z$ and $z^{\prime}$ on the two spheres:

$$
\lambda^{2} h_{3}(z) h_{3}\left(z^{\prime}\right)=q,
$$

inside open neighborhoods of $z=z_{3}$ and $z^{\prime}=z_{3}^{\prime}$. Since the glued Riemann surface is a sphere with four punctures, we can introduce a global coordinate $y$ on the four punctured sphere. If the $h_{i}$ 's had been $\mathrm{SL}(2, \mathrm{C})$ transformations as in footnote 7 then $y$ could be taken to be either $z$ or $z^{\prime}$ or related to these by an $\mathrm{SL}(2, \mathrm{C})$ transformation, but in general the relation between $y$ and the original coordinates $z, z^{\prime}$ is more complicated. By an $\mathrm{SL}(2, \mathrm{C})$ transformation we can ensure that in the $y$ plane the original punctures at $z=0$ and $\infty$ are located at $y=0$ and $\infty$, and the puncture at $z^{\prime}=\infty$ is located at $y=1$. We shall denote by $\sigma$ the location of the puncture at $z^{\prime}=0$ in the $y$ plane. It takes the form of a function of $q / \lambda^{2}$ due to (3.9). Therefore in the $y$ plane the four punctures are located at:

$$
y_{1}=0, \quad y_{2}=\infty, \quad y_{3}=1, \quad y_{4}=\sigma=g\left(q / \lambda^{2}\right),
$$

\footnotetext{
${ }^{7} \mathrm{~A}$ particular choice of local coordinates satisfying these relations is given by [44] $h_{1}(z)=z /(z-2)$, $h_{2}(z)=1 /(2 z-1)$ and $h_{3}(z)=(1-z) /(1+z)$, but we shall proceed without committing ourselves to any particular choice.
} 
for some function $g . y_{4}=\sigma$ is a holomorphic function of $q$ for $|q|<1$, but we shall not make use of this information in an essential way. We also define $v_{1}, v_{2}, v_{3}$ and $v_{4}$ to be the original local coordinates $w_{1}, w_{2}, w_{1}^{\prime}$ and $w_{2}^{\prime}$, but now expressed as function of $y$. These take the form

$$
v_{i}=\lambda \hat{h}_{i}\left(y ; q / \lambda^{2}\right),
$$

for some functions $\hat{h}_{i}$. The overall multiplicative factors of $\lambda$ come from the multiplicative factors of $\lambda$ in the definitions of $w_{1}, w_{2}, w_{1}^{\prime}$ and $w_{2}^{\prime}$ while the dependence on $q / \lambda^{2}$ enters through (3.9).

It follows from (3.9) that as $q \rightarrow 0$, the four punctured sphere degenerates into two spheres, with the original punctures at $z=0$ and $z=\infty$ on one sphere and the original punctures at $z^{\prime}=0$ and $z^{\prime}=\infty$ on the other sphere. In the $y$ plane this will correspond to the punctures at $\sigma$ and 1 coming together. To find the behavior of $g\left(q / \lambda^{2}\right)$ for small $q$, we note that for small $q / \lambda^{2}$ we can take $z, z^{\prime}$ close to 1 and express the relation (3.9) as

$$
\lambda^{2}\left(h_{3}^{\prime}(1)\right)^{2}(z-1)\left(z^{\prime}-1\right)=q .
$$

Since in this case the local coordinates are related to global coordinates $z$ and $z^{\prime}$ via $\mathrm{S}(2, \mathrm{C})$ transformation, we can identify $z$ (and $z^{\prime}$ ) with $y$ up to $\mathrm{SL}(2, \mathrm{C})$ transformation. Now under the identification (3.12) the puncture at $z^{\prime}=\infty$ is mapped to $z=1$ and the puncture $z^{\prime}=0$ is mapped to $z=1-q \lambda^{-2}\left(h_{3}^{\prime}(1)\right)^{-2}$. Therefore we can make the identification $y=z$ and $y_{4} \equiv \sigma=1-q \lambda^{-2}\left(h_{3}^{\prime}(1)\right)^{-2}$. Comparing this with (3.10) we get

$$
g\left(q / \lambda^{2}\right) \simeq 1-q \lambda^{-2}\left(h_{3}^{\prime}(1)\right)^{-2}+\mathcal{O}\left(q^{2} / \lambda^{4}\right) .
$$

Therefore we have

$$
g(0)=1, \quad g^{\prime}(0)=-\left(h_{3}^{\prime}(1)\right)^{-2} .
$$

The region $|q| \leq 1$ corresponds to a neighborhood of the point 1 in the $\sigma$ plane. In string field theory, if we use the local coordinates given in (3.2) to define the 3-point interaction vertex, then the family of four punctured spheres (3.10) corresponding to $|q| \leq 1$, and the local coordinates given in (3.11), describe the contribution to the four point Green's function due to 's-channel diagrams'. In this the external states represented by the vertex operators inserted at the punctures at $y_{1}$ and $y_{2}$ merge to form an intermediate state which then splits into the states represented by the vertex operators inserted at the punctures $y_{3}$ and $y_{4}$.

Contribution from the $u$-channel diagram is obtained by exchanging 1 and 3 . Denoting the global coordinate on the plane by $\tilde{y}$, we get, by exchanging 1 and 3 in (3.10),

$$
\tilde{y}_{1}=1, \quad \tilde{y}_{2}=\infty, \quad \tilde{y}_{3}=0, \quad \tilde{y}_{4}=g\left(q / \lambda^{2}\right),
$$

and the local coordinates at the punctures are

$$
v_{1}=\lambda \hat{h}_{3}\left(\tilde{y} ; q / \lambda^{2}\right), \quad v_{2}=\lambda \hat{h}_{2}\left(\tilde{y} ; q / \lambda^{2}\right), \quad v_{3}=\lambda \hat{h}_{1}\left(\tilde{y} ; q / \lambda^{2}\right), \quad v_{4}=\lambda \hat{h}_{4}\left(\tilde{y} ; q / \lambda^{2}\right) .
$$

We now make a change of variable

$$
y=1-\tilde{y},
$$


so that in the $y$ coordinate system the locations of the punctures are given by

$$
y_{1}=0, \quad y_{2}=\infty, \quad y_{3}=1, \quad y_{4}=\sigma=1-g\left(q / \lambda^{2}\right),
$$

and the local coordinates around the punctures are given by,

$$
\begin{array}{ll}
v_{1}=\lambda \hat{h}_{3}\left(1-y ; q / \lambda^{2}\right), & v_{2}=\lambda \hat{h}_{2}\left(1-y ; q / \lambda^{2}\right), \\
v_{3}=\lambda \hat{h}_{1}\left(1-y ; q / \lambda^{2}\right), & v_{4}=\lambda \hat{h}_{4}\left(1-y ; q / \lambda^{2}\right) .
\end{array}
$$

Contribution from the $t$-channel diagram is given by exchanging 2 and 3 in the $s$ channel contribution. Denoting the global coordinate on the plane by $\hat{y}$, we get

$$
\begin{array}{llll}
\hat{y}_{1}=0, & \hat{y}_{2}=1, & \hat{y}_{3}=\infty, & \hat{y}_{4}=g\left(q / \lambda^{2}\right), \\
v_{1}=\lambda \hat{h}_{1}\left(\hat{y} ; q / \lambda^{2}\right), & v_{2}=\lambda \hat{h}_{3}\left(\hat{y} ; q / \lambda^{2}\right), & v_{3}=\lambda \hat{h}_{2}\left(\hat{y} ; q / \lambda^{2}\right), & v_{4}=\lambda \hat{h}_{4}\left(\hat{y} ; q / \lambda^{2}\right) .
\end{array}
$$

We make a change of variables

$$
y=\frac{\hat{y}}{\hat{y}-1}, \quad \hat{y}=\frac{y}{y-1} .
$$

In this coordinate system the punctures are located at

$$
y_{1}=0, \quad y_{2}=\infty, \quad y_{3}=1, \quad y_{4}=\sigma=\frac{g\left(q / \lambda^{2}\right)}{g\left(q / \lambda^{2}\right)-1},
$$

and the local coordinates are given by

$$
\begin{array}{ll}
v_{1}=\lambda \hat{h}_{1}\left(y /(y-1) ; q / \lambda^{2}\right), & v_{2}=\lambda \hat{h}_{3}\left(y /(y-1) ; q / \lambda^{2}\right), \\
v_{3}=\lambda \hat{h}_{2}\left(y /(y-1) ; q / \lambda^{2}\right), & v_{4}=\lambda \hat{h}_{4}\left(y /(y-1) ; q / \lambda^{2}\right) .
\end{array}
$$

Using (3.13) we see that in the $y_{4}=\sigma$ plane, the region $|q| \leq 1$ in (3.10), (3.17) and (3.20) map respectively to some regions around 1,0 and $\infty$ which we shall denote by $\mathcal{R}_{s}, \mathcal{R}_{u}$ and $\mathcal{R}_{t}$. Integrals over these regions describe the contributions from $s, u$ and $t$-channel Feynman diagrams. The rest of the region in the $\sigma$ plane, which we shall call $\mathcal{R}$, must come from the elementary four point interaction vertex.

In order to define the four point interaction vertex for off-shell string states, we need to look for local coordinates of the form

$$
v_{i}=\lambda \tilde{h}_{i}(y ; \sigma), \quad 1 \leq i \leq 4, \quad \sigma \in \mathcal{R},
$$

satisfying the following conditions:

1. $v_{i}$ must vanish as $y \rightarrow y_{i}$ :

$$
\tilde{h}_{i}\left(y_{i} ; \sigma\right)=0 .
$$

2. $\tilde{h}_{i}(y ; \sigma)$ must match the results (3.11), (3.18) and (3.21) of $s, u$ and $t$ channel diagrams when $\sigma$ takes value at the common boundary of $\mathcal{R}$ and $\mathcal{R}_{s}, \mathcal{R}$ and $\mathcal{R}_{u}$ and $\mathcal{R}$ and $\mathcal{R}_{t}$ respectively, corresponding to setting $q=e^{-i \theta}$ in (3.15), (3.17) and (3.20). 
3. $v_{i}(\sigma)$ must obey permutation symmetry. This means the following. Let us suppose that we exchange the punctures $k$ and $\ell$ for some fixed $k$ and $\ell$. This exchanges $y_{k}$ with $y_{\ell}$ and also $v_{k}$ with $v_{\ell}$. Now by an $\operatorname{SL}(2, \mathrm{C})$ transformation we can bring the locations of the punctures 1,2 and 3 to their original values $0, \infty$ and 1 , but in that process $y_{4}=\sigma$ will typically map to a different point $\sigma^{\prime}$ and the local coordinates $v_{i}$, expressed in terms of the new complex coordinate, which we shall still denote by $y$, will change to $v_{i}^{\prime}$. The requirement that we would like to impose is that $v_{i}^{\prime}$ should agree with $v_{i}$ at $\sigma^{\prime}$. This will guarantee that interaction vertex is invariant under permutation of the external states.

While it will be nice to have an interaction vertex of this type, the third condition is not strictly necessary. Given a choice of local coordinates that satisfy conditions 1 and 2 but not 3 , we can define the interaction vertex by taking average of the result computed from this choice of local coordinates and the images of these local coordinates under permutation.

Similar procedure can be followed for the construction of the local coordinates needed for higher point interaction vertices of string field theory.

We shall end this section with a few comments on the parameter $\lambda$.

1. While we expect the on-shell amplitudes to be independent of the parameter $\lambda$ (and the functions $h_{i}$ ), the off-shell amplitudes depend on $\lambda$. Since $d w_{i} / d z$ computed from (3.2) is proportional to $\lambda$, the off-shell amplitude of a mode with $L_{0}+\bar{L}_{0}$ eigenvalue $h$ is proportional to $\left|d w_{i} / d z\right|^{-h} \sim \lambda^{-h}$. Therefore for large $\lambda$ the contribution from the modes with large $L_{0}+\bar{L}_{0}$ eigenvalue is highly suppressed. This suppression factor in turn makes the sum over massive states in the intermediate state finite even though the number of such modes has a Hagedorn growth.

2. As can be seen from (3.10), (3.17) and (3.20), for large $\lambda$ the $s, u$ and $t$ channel diagrams cover small regions of the $\sigma \equiv y_{4}$ plane around 1,0 and $\infty$ respectively, given by the $|q| \leq 1$ regions in (3.10), (3.17) and (3.20). Therefore most of the integration over $\sigma=y_{4}$ is generated by the four point interaction vertex. This is related to the fact that the regions $\left|w_{i}\right| \leq 1$ in (3.2) cover small regions in the $z$ plane.

3. Note that $q$ always appears in the combination $q / \lambda^{2}$. This means in particular that if we change the cut-off $\Lambda$ in (1.3) and simultaneously change $\lambda$ so that $\lambda^{2} e^{\Lambda}$ remains fixed, then the cut-off $|q|=e^{-s} \geq e^{-\Lambda}$ remains unchanged in the $\sigma=y_{4}$ plane. Using this we can set $\Lambda=0$ by scaling $\lambda$ by $e^{\Lambda / 2}$. In this case the first term in (1.3) vanishes and the second term becomes (1.1). Therefore use of (1.3) to deal with the negative $\left(L_{0}+\bar{L}_{0}\right)$ states is equivalent to the use of (1.1), with a rescaled value of $\lambda$.

\subsection{Locations of picture changing operators}

For constructing the interaction vertices of superstring theories, we also need to make a choice of PCO locations. We shall now briefly describe the procedure. For simplicity we focus on the heterotic string theory. For type II string theories we have to repeat the procedure for the left and the right-moving sectors of the world-sheet theory separately. 
We begin with the cubic interaction vertex. There are two types of vertices: R-R-NS and NS-NS-NS. For the R-R-NS vertex the total picture number of the vertex operators is -2 and we do not need to add any PCO. Therefore the vertex is the same as that in bosonic string theory. For the NS-NS-NS vertex, the picture numbers of the three NS-sector vertex operators add up to -3 and we need to insert one PCO. The location of the PCO needs to be invariant under permutation symmetry, i.e. under the $\mathrm{SL}(2, \mathrm{C})$ transformations that exchange the positions 0,1 and $\infty$ of the vertex operators. One can either try to choose the location to be invariant under this symmetry group, or pick an arbitrary location and average over all its images. It is easy to verify that there is no single location that is invariant under the subgroup of $\operatorname{SL}(2, \mathrm{C})$ generating arbitrary permutation of 0,1 and $\infty$. The brute force way will be to choose an arbitrary point $p_{1}$ and its images $p_{2}, \cdots p_{6}$ under the symmetry generated by $z \rightarrow 1-z$ and $z \rightarrow z /(z-1)$ and take the PCO insertion to be

$$
\frac{1}{6} \sum_{i=1}^{6} \mathcal{X}\left(p_{i}\right) \text {. }
$$

A more economical procedure will be to pick a pair of points, each of which is invariant under the subgroup that generates cyclic permutation of 0,1 and $\infty$, and the pair gets exchanged under a $Z_{2}$ transformations that exchange 0 and 1 , leaving $\infty$ fixed. These pair of points are:

$$
p_{1}=\frac{1}{2}+i \frac{\sqrt{3}}{2}, \quad p_{2}=\frac{1}{2}-i \frac{\sqrt{3}}{2} .
$$

We can now insert $\left(\mathcal{X}\left(p_{1}\right)+\mathcal{X}\left(p_{2}\right)\right) / 2$ to get a symmetric 3 -point interaction vertex. In the following we shall denote the PCO location for a 3-point interaction vertex by $p$, keeping in mind that $p$ may stand for averages over several locations.

Let us now consider the contribution to the 4-point amplitudes associated with $s, t$ and $u$ channel diagrams. First consider the case of four R-sector external states. In this case the intermediate state in each channel is an NS sector state. No PCO is needed at any stage of this calculation, reducing the analysis to that of bosonic string field theory.

Next consider the case of four NS sector external states. In the $s$-channel diagrams the PCOs are located at

$$
z=p, \quad z^{\prime}=p
$$

where $p$ corresponds to average of several insertions as in (3.24). Their images $W_{1}$ and $W_{2}$ in the $y$ plane may be found using the known map between $z, z^{\prime}$ and $y$. The information that will be useful to us later is that in the small $q$ or large $\lambda$ limit, one of the PCO's (say $W_{1}$ ) is finite distance away from the punctures 1 and $\sigma$ while the other one (say $W_{2}$ ) is close to the punctures 1 and $\sigma$. For $t$ and $u$ channel diagrams the locations of the PCOs are related to $W_{1}, W_{2}$ by the transformations $y \rightarrow y /(y-1)$ and $y \rightarrow(1-y)$ respectively.

While constructing the 4-point interaction vertex of four NS sector states, one can choose the local coordinates at the punctures for $\sigma \in \mathcal{R}$ as in the case of bosonic string theory, but we also need to fix the location of two PCOs as a function of the modulus $\sigma$. The PCO locations $\left(W_{1}, W_{2}\right)$ on $\partial \mathcal{R}_{s}, \partial \mathcal{R}_{u}$ and $\partial \mathcal{R}_{t}$ induced from the s, u and tchannel diagrams provide appropriate boundary condition that the PCO locations must 
satisfy. They also must respect the permutation symmetry. This can be achieved by brute force by choosing one configuration satisfying the boundary condition and then averaging over all its images under permutation. However, one may be able to reduce the number of configurations to average over by making judicious choice of the initial configuration so that it is invariant under a subgroup of the permutation group. Other than these constraints the PCO locations on the four punctured sphere can be chosen arbitrarily for $\sigma \in \mathcal{R}$. If necessary (e.g. to avoid collision with each other) we can even allow the PCO locations to jump discontinuously across codimension one subspaces inside $\mathcal{R}$ and add correction terms $[45,46]$, so there is no obstruction to choosing the PCOs satisfying the desired conditions.

The case of two NS and two R vertex operators can be analyzed similarly. In this case in the channel where an $\mathrm{R}$ sector state propagates as intermediate state, the PCO insertion comes from the factor of $\mathcal{G}$ in the $\mathrm{R}$ sector propagator (2.22). Therefore the PCO insertion involves an average not over discrete number of possibilities but a continuous set of possibilities. As before, for $\sigma \in \mathcal{R}$ the PCO locations can be chosen arbitrarily subject to the boundary conditions on $\partial \mathcal{R}_{s}, \partial \mathcal{R}_{t}$ and $\partial \mathcal{R}_{u}$ and the symmetry requirement (which in this case corresponds to the exchange of the two NS punctures and (independently) the two $\mathrm{R}$ punctures.

\section{Warm up with four point amplitude}

We shall now show how bosonic string field theory can be used to compute the four point amplitude over the full range of external momenta without any need for analytic continuation. In section 4.1 we shall outline the general procedure for determining the boundary term that arises when we use (1.3) to deal with the contribution from states with negative $L_{0}+\bar{L}_{0}$ eigenvalue. In section 4.2 we shall use this to express the four tachyon amplitude as an integral over the world-sheet that does not have any divergence.

\subsection{A concise description of the boundary terms}

Let us consider either the s, t or u-channel Feynman diagram of string field theory four point amplitude. Each of these diagrams has a single propagator. Using the representation (1.3) of the propagator we can express the contribution to the diagram from the first term on the right hand side of (1.3) as a two dimensional integral over the complex variable $q$, with the integrand given by ${ }^{8}$

$$
\mathcal{I}^{(0)}=d q \wedge d \bar{q} \sum_{i} A_{i} q^{-1+\gamma_{i}} \bar{q}^{-1+\delta_{i}}
$$

where $\gamma_{i}$ and $\delta_{i}$ are the $L_{0}$ and $\bar{L}_{0}$ eigenvalues of the $i$-th internal state and the constants $A_{i}$ are given by the products of the vertices of the Feynman diagram. We shall work with

\footnotetext{
${ }^{8}$ Even though string field theory contains only level matched states, in establishing the correspondence between the string field theory amplitudes and world-sheet amplitudes, it is more convenient to include in the interaction vertices non-level matched external states and impose the level matching condition by integration over the phase of the sewing parameter $q$. This is the reason why (4.1) contains terms with $\gamma_{i} \neq \delta_{i}$. The integration over the phase of $q$ implements the $\gamma_{i}=\delta_{i}$ constraint.
} 
generic external momenta so that there are no terms with integer $\gamma_{i}, \delta_{i}$. On the other hand the contribution to the Feynman diagram from the second term on the right hand side of (1.3) may be represented as a boundary integral of the form:

$$
\begin{aligned}
\mathcal{B} & =\int_{|q|=e^{-\Lambda}} \mathcal{I}^{(1)}, \\
\mathcal{I}^{(1)} & =-d q \sum_{i} A_{i} \delta_{i}^{-1} q^{-1+\gamma_{i}} \bar{q}^{\delta_{i}} .
\end{aligned}
$$

Note that $\mathcal{I}^{(1)}$ satisfies

$$
d \mathcal{I}^{(1)}=\mathcal{I}^{(0)} .
$$

The choice (4.3) of $\mathcal{I}^{(1)}$ is not unique since we could have taken this to be proportional to $d \bar{q}$ or a linear combination of $d q$ and $d \bar{q}$ which all satisfy (4.4). This ambiguity corresponds to the freedom of adding exact forms to $\mathcal{I}^{(1)}$.

By the standard procedure in string field theory illustrated in section 3.1 one can convert the integration over the parameters $q, \bar{q}$ associated with the propagators to the integration over the standard world-sheet variables $\sigma, \bar{\sigma}$ denoting the world-sheet coordinate of one of the vertex operators, keeping fixed the positions of the other three vertex operators. In the convention of section 3.1 the region $|q| \leq 1$ gets mapped to the regions $\mathcal{R}_{s}, \mathcal{R}_{t}$ and $\mathcal{R}_{u}$ in the $\sigma$-plane for the s, t and u-channel diagrams. Let us suppose that the region $|q| \leq e^{-\Lambda}$ gets mapped to the regions $\widetilde{\mathcal{R}}_{s}, \widetilde{\mathcal{R}}_{t}$ and $\widetilde{\mathcal{R}}_{u}$ for these diagrams. Then the boundary integrals (4.2) will run over $\partial \widetilde{\mathcal{R}}_{s}, \partial \widetilde{\mathcal{R}}_{t}$ and $\partial \widetilde{\mathcal{R}}_{u}$. On the other hand the bulk integral, after combining the contributions from the s, t and u-channel Feynman diagrams and the diagram involving the four point interaction vertex, will run over the full complex $\sigma$-plane except the excluded regions $\widetilde{\mathcal{R}}_{s}, \widetilde{\mathcal{R}}_{t}$ and $\widetilde{\mathcal{R}}_{u}$.

(4.4) gives a simple way of determining $\mathcal{I}^{(1)}$ from the original integrand $\mathcal{I}^{(0)}$ without knowing the relation between $q$ and $\sigma$. Let us suppose that $\sigma$ approaches $\sigma_{0}$ as $q$ approaches 0 . Here $\sigma_{0}$ is the location of one of the other vertex operators. Then near $q=0$ the relation between $\sigma$ and $q$ takes the form

$$
q=f(\sigma)=\sum_{n \geq 1} a_{n}\left(\sigma-\sigma_{0}\right)^{n} .
$$

In terms of the variables $\sigma$ and $\bar{\sigma}$ we can express (4.1) as

$$
\mathcal{I}^{(0)}=d \sigma \wedge d \bar{\sigma} \sum_{j} B_{j}\left(\sigma-\sigma_{0}\right)^{-1+\alpha_{j}}\left(\bar{\sigma}-\bar{\sigma}_{0}\right)^{-1+\beta_{j}},
$$

where $B_{j}$ are new coefficients and $\alpha_{j}, \beta_{j}$ take values that differ from $\gamma_{j}, \delta_{j}$ at most by integers. We can now solve (4.4) to get

$$
\mathcal{I}^{(1)}=-d \sigma \sum_{j} B_{j} \beta_{j}^{-1}\left(\sigma-\sigma_{0}\right)^{-1+\alpha_{j}}\left(\bar{\sigma}-\bar{\sigma}_{0}\right)^{\beta_{j}} \quad \text { near } \sigma=\sigma_{0},
$$

up to closed differential forms. Now one can show that a closed differential form with an expansion in non-integer powers of $\left(\sigma-\sigma_{0}\right)$ and $\left(\bar{\sigma}-\bar{\sigma}_{0}\right)$ is also exact, with an expansion 
involving non-integer powers of $\left(\sigma-\sigma_{0}\right)$ and $\left(\bar{\sigma}-\bar{\sigma}_{0}\right)$. Therefore $\mathcal{I}^{(1)}$ given in (4.7) differs from the one in (4.3) at most by an exact form and its integral over the cycle $|q|=e^{-\Lambda}$ gives the same result as that of (4.3). When $\sigma_{0}=\infty$, one has to use a slight variant of this procedure, with $\mathcal{I}^{(0)}$ expanded as $d \sigma \wedge d \bar{\sigma} \sum_{j} B_{j} \sigma^{-1-\alpha_{j}} \bar{\sigma}^{-1-\beta_{j}}$ and $\mathcal{I}^{(1)}$ given by $d \sigma \sum_{j} B_{j} \beta_{j}^{-1} \sigma^{-1-\alpha_{j}} \bar{\sigma}^{-\beta_{j}}$.

In this procedure the only part that requires the knowledge of the relation between $q$ and $\sigma$ is the curve $\mathcal{C}=\partial \widetilde{\mathcal{R}}_{u}, \partial \widetilde{\mathcal{R}}_{s}$ or $\partial \widetilde{\mathcal{R}}_{u}$ - the image of the curve $|q|=e^{-\Lambda}$ in the $\sigma$ plane. However we shall now show that the sum of the bulk and the boundary terms is independent of the form of $\mathcal{C}$. For this let us recall that the bulk integration runs over the region outside the curve $\mathcal{C}$ in the $\sigma$ plane. Therefore if we change $\mathcal{C}$ to $\mathcal{C}^{\prime}$, the bulk integration region will change by a region $\mathcal{D}$ with $\partial \mathcal{D}=\mathcal{C}-\mathcal{C}^{\prime}$. The net change in the bulk and the boundary integral is given by

$$
\int_{\mathcal{D}} \mathcal{I}^{(0)}+\int_{\mathcal{C}^{\prime}-\mathcal{C}} \mathcal{I}^{(1)}=\int_{\mathcal{D}} d \mathcal{I}^{(1)}-\int_{\partial \mathcal{D}} \mathcal{I}^{(1)}=0
$$

This shows that even the knowledge of $\mathcal{C}$ is not needed and (4.7) gives us complete information about the boundary term in terms of the bulk integrand (4.6). This in turn shows that in order to evaluate the sum of the boundary terms and the bulk integral that defines the amplitude, we do not need any knowledge of the local coordinates used in defining the interaction vertices of string field theory.

While for computing amplitudes with generic external momenta, we can assume that $\gamma_{j}, \delta_{j}$ and hence $\alpha_{j}, \beta_{j}$ are not integers, in section 7 , where we analyze the effect of marginal deformations, we shall encounter situations where $\alpha_{j}, \beta_{j}$ are integers. In this case there may be an additional contribution to the boundary term involving the integral of a one form that is closed but not exact:

$$
-\int_{\mathcal{C}} d \sigma K\left(\sigma-\sigma_{0}\right)^{-1}
$$

Here $K$ is a constant. As we shall see in section 7, possible additional contributions from such terms can be calculated by working directly with (4.1).

\subsection{Four tachyon amplitude}

We shall now apply the procedure described in section 4.1 to give an expression for the four tachyon amplitude as a world-sheet integral that does not have any divergence. First let us describe the conventional world-sheet computation in the $\alpha^{\prime}=1$ unit. Let us denote by $k_{1}, k_{2}, k_{3}$ and $k_{4}$ the momenta of the tachyons, all counted as positive if ingoing, and satisfying the on-shell condition

$$
k_{i}^{2}=-m^{2}=4
$$

where $m^{2}$ is the mass ${ }^{2}$ of the tachyon. Then according to (2.15), the amplitude takes the form

$$
A=\frac{1}{2 \pi i} \int d^{2} \sigma\left\langle c \bar{c} e^{i k_{1} \cdot X}\left(y_{1}, \bar{y}_{1}\right) c \bar{c} e^{i k_{2} \cdot X}\left(y_{2}, \bar{y}_{2}\right) c \bar{c} e^{i k_{3} \cdot X}\left(y_{3}, \bar{y}_{3}\right) e^{i k_{4} \cdot X}(\sigma, \bar{\sigma})\right\rangle,
$$


where $y_{1}, y_{2}, y_{3}$ are arbitrary fixed points, which we can take to be $0, \infty$ and 1 respectively, and

$$
d^{2} \sigma \equiv d \sigma \wedge d \bar{\sigma}
$$

After evaluating the correlation function using (2.1) the result takes the form:

$$
A=-\frac{1}{2 \pi i} \int d^{2} \sigma|\sigma|^{k_{1} \cdot k_{4}}|\sigma-1|^{k_{3} \cdot k_{4}}
$$

up to overall momentum conserving delta function. Defining

$$
\begin{aligned}
& s=-\left(k_{3}+k_{4}\right)^{2}=-8-2 k_{3} \cdot k_{4}, \quad t=-\left(k_{2}+k_{4}\right)^{2}=-8-2 k_{2} \cdot k_{4}, \\
& u=-\left(k_{1}+k_{4}\right)^{2}=-8-2 k_{1} \cdot k_{4},
\end{aligned}
$$

we can express (4.13) as

$$
A=-\frac{1}{2 \pi i} \int d^{2} \sigma|\sigma|^{-u / 2-4}|\sigma-1|^{-s / 2-4}=-\frac{1}{2 \pi i} \int d^{2} \sigma|\sigma|^{-\left(u-m^{2}\right) / 2-2}|\sigma-1|^{-\left(s-m^{2}\right) / 2-2} .
$$

This integral has divergences from the region $\sigma \rightarrow 0, \sigma \rightarrow 1$ and $\sigma \rightarrow \infty$ if, respectively,

$$
u \geq m^{2}, \quad s \geq m^{2}, \quad t \geq m^{2} .
$$

Therefore the divergences appear whenever $s, t$ or $u$ exceeds the threshold of production of a tachyonic particle in the intermediate state. Precisely in these domains the intermediate tachyon state propagating in the $s, t$ and $u$-channel respectively has negative $L_{0}+\bar{L}_{0}$ eigenvalue and the right hand side of (1.2) diverges. Conventionally one first defines this integral in the region where it converges and then goes out of this region via analytic continuation. This leads to the result:

$$
A=\frac{\Gamma(-1-s / 4) \Gamma(-1-t / 4) \Gamma(-1-u / 4)}{\Gamma(2+s / 4) \Gamma(2+t / 4) \Gamma(2+u / 4)} .
$$

Our goal will be to modify (4.15) suitably by drawing insights from string field theory so that we can evaluate the integral without having to invoke analytic continuation. For generic $s, t$ and $u$ we can invoke the results of section 4.1 to arrive at such an expression. We cut out small regions $\widetilde{\mathcal{R}}_{s}, \widetilde{\mathcal{R}}_{u}$ and $\widetilde{\mathcal{R}}_{t}$ around 1,0 and $\infty$ respectively, and denote by $\widetilde{\mathcal{R}}$ the left over region in the complex plane. Then we can express the amplitude as

$$
A=-\frac{1}{2 \pi i} \int_{\widetilde{\mathcal{R}}} d^{2} \sigma|\sigma|^{-u / 2-4}|\sigma-1|^{-s / 2-4}+\mathcal{B}_{s}+\mathcal{B}_{t}+\mathcal{B}_{u}
$$

where the last three terms are boundary terms which can be evaluated from the bulk integrand using (4.7) as follows. Suppose that near $\sigma=0$

$$
-\frac{1}{2 \pi i}|\sigma|^{-u / 2-4}|\sigma-1|^{-s / 2-4}=\sum_{i} C_{i} \sigma^{-1+\alpha_{i}} \bar{\sigma}^{-1+\beta_{i}},
$$

where $\alpha_{i}, \beta_{i}$ are given by $-u / 4-1$ plus non-negative integers and $C_{i}$ are constants. Then (4.7) gives

$$
\mathcal{B}_{u}=-\int_{\partial \widetilde{\mathcal{R}}_{u}} \sum_{i} C_{i} \frac{1}{\beta_{i}} d \sigma \sigma^{\alpha_{i}-1} \bar{\sigma}^{\beta_{i}}
$$


Similarly if we denote the power series expansion of the integrand in the region around $\sigma=1$ as

$$
-\frac{1}{2 \pi i}|\sigma|^{-u / 2-4}|\sigma-1|^{-s / 2-4}=\sum_{i} C_{i}^{\prime}(\sigma-1)^{\alpha_{i}^{\prime}-1}(\bar{\sigma}-1)^{\beta_{i}^{\prime}-1},
$$

then the required boundary terms on $\partial \widetilde{\mathcal{R}}_{s}$ can be expressed as

$$
\mathcal{B}_{s}=-\int_{\partial \widetilde{\mathcal{R}}_{s}} d \sigma \sum_{i} C_{i}^{\prime} \frac{1}{\beta_{i}^{\prime}}(\sigma-1)^{\alpha_{i}^{\prime}-1}(\bar{\sigma}-1)^{\beta_{i}^{\prime}}
$$

Finally if we expand the integrand in a power series expansion in $|\sigma|^{-1}$ around $\sigma=\infty$ :

$$
-\frac{1}{2 \pi i}|\sigma|^{-u / 2-4}|\sigma-1|^{-s / 2-4}=\sum_{i} C_{i}^{\prime \prime} \sigma^{-\alpha_{i}^{\prime \prime}-1} \bar{\sigma}^{-\beta_{i}^{\prime \prime}-1}
$$

then the required boundary terms on $\partial \widetilde{\mathcal{R}}_{t}$ are given by

$$
\mathcal{B}_{t}=\int_{\partial \widetilde{\mathcal{R}}_{t}} d \sigma \sum_{i} C_{i}^{\prime \prime} \frac{1}{\beta_{i}^{\prime \prime}} \sigma^{-\alpha_{i}^{\prime \prime}-1} \bar{\sigma}^{-\beta_{i}^{\prime \prime}}
$$

The expressions for $\mathcal{B}_{u}, \mathcal{B}_{s}$ and $\mathcal{B}_{t}$ given above involve infinite sum over states. However the sum converges and there is no difficulty in numerical evaluation of this expression. We have tested (4.18) by numerically evaluating this and comparing this with the exact result (4.17) in the regime in $(s, t, u)$ space where the original integral (4.15) diverges. By taking the contours around 0 and 1 to be sufficiently small and the contour around $\infty$ to be sufficiently large, we can ensure that only finite number of terms with negative $\alpha_{i}+\beta_{i}$, $\alpha_{i}^{\prime}+\beta_{i}^{\prime}$ and $\alpha_{i}^{\prime \prime}+\beta_{i}^{\prime \prime}$ give appreciable contribution to (4.20), (4.22) and (4.24).

As a simple example we can consider the domain $s<-4, t<-4$ and $-4<u<-2$. In this case the divergences in the integral (4.19) will come only from the region near $\sigma=0$. Therefore we can take the limit in which $\widetilde{\mathcal{R}}_{s}$ shrinks to $\sigma=1$ and $\widetilde{\mathcal{R}}_{t}$ recedes to infinity, making $\mathcal{B}_{s}$ and $\mathcal{B}_{t}$ vanish. To determine $\mathcal{B}_{u}$ we expand the bulk integrand in a power series in $\sigma$ to get

$$
\begin{aligned}
-\frac{1}{2 \pi i}|\sigma|^{-u / 2-4}|\sigma-1|^{-s / 2-4}= & -\frac{1}{2 \pi i}|\sigma|^{-u / 2-4}\left\{1+\left(\frac{s}{4}+2\right)(\sigma+\bar{\sigma})+\left(\frac{s}{4}+2\right)^{2} \sigma \bar{\sigma}\right. \\
& \left.+\frac{1}{2}\left(\frac{s}{4}+2\right)\left(\frac{s}{4}+3\right)\left(\sigma^{2}+\bar{\sigma}^{2}\right)+\cdots\right\} .
\end{aligned}
$$

This gives, from (4.20),

$$
\begin{aligned}
\mathcal{B}_{u}= & \frac{1}{2 \pi i} \int_{\partial \widetilde{\mathcal{R}}_{u}} d \sigma|\sigma|^{-u / 2-4}\left[-\frac{4}{u+4} \bar{\sigma}-\left(\frac{s}{4}+2\right) \frac{4}{u+4} \bar{\sigma} \sigma-\left(\frac{s}{4}+2\right) \frac{4}{u} \bar{\sigma}^{2}\right. \\
& \left.-\left(\frac{s}{4}+2\right)^{2} \frac{4}{u} \sigma \bar{\sigma}^{2}-\frac{1}{2}\left(\frac{s}{4}+2\right)\left(\frac{s}{4}+3\right)\left(\frac{4}{u+4} \sigma^{2} \bar{\sigma}+\frac{4}{u-4} \bar{\sigma}^{3}\right)+\cdots\right] .
\end{aligned}
$$

Since (4.18) has been shown to be independent of the choice of contours $\partial \widetilde{\mathcal{R}}_{u}, \partial \widetilde{\mathcal{R}}_{s}$ and $\partial \widetilde{\mathcal{R}}_{t}$, we can take $\widetilde{\mathcal{R}}_{u}$ to be a rectangular region around the origin. As the size of $\widetilde{\mathcal{R}}_{u}$ shrinks, contribution to $\mathcal{B}_{u}$ from the first term inside the square bracket increases and the 
other terms decrease for $-4<u<-2$. By taking the contour to be sufficiently small, we can ignore the contribution from all terms other than the first term in the square bracket. Adding this to the bulk integral given by the first term in (4.18), with $\widetilde{\mathcal{R}}$ given by the complement of $\widetilde{\mathcal{R}}_{u}$ in the whole complex plane, we can evaluate the full amplitude. With relatively little effort we can reduce the error in computation - measured by comparing the result to the exact expression given in (4.17) - to less than .1\%. The error comes from the higher order terms inside the square bracket in (4.26). We can further reduce the error either by including these terms or by shrinking the size of $\widetilde{\mathcal{R}}_{u}$, at the same time making sure that the error in the numerical evaluation of the integrals are not significant. ${ }^{9}$

We shall now give an alternate expression that does not require infinite sum. This requires taking the limit in which the regions $\widetilde{\mathcal{R}}_{s}, \widetilde{\mathcal{R}}_{t}$ and $\widetilde{\mathcal{R}}_{u}$ shrink to points $1, \infty$ and 0 respectively. In this limit only the terms with negative $\alpha_{i}+\beta_{i}, \alpha_{i}^{\prime}+\beta_{i}^{\prime}$ and $\alpha_{i}^{\prime \prime}+\beta_{i}^{\prime \prime}$ survive in the boundary terms. We now use

$$
\frac{1}{\beta_{i}} \int_{\partial \widetilde{\mathcal{R}}_{u}} d \sigma \sigma^{\alpha_{i}-1} \bar{\sigma}^{\beta_{i}}=\int_{\widetilde{\mathcal{R}}_{u}^{c}} d^{2} \sigma \sigma^{\alpha_{i}-1} \bar{\sigma}^{\beta_{i}-1}, \quad \text { for } \alpha_{i}+\beta_{i}<0,
$$

where $\widetilde{\mathcal{R}}_{u}^{c}$ is the complement of the region $\widetilde{\mathcal{R}}_{u}$. In the limit when $\widetilde{\mathcal{R}}_{u}$ shrinks to a point $\widetilde{\mathcal{R}}_{u}^{c}$ will expand into the whole complex plane, but we do not want to take this limit yet since the integral (4.27) will diverge in this limit. Using (4.27) and analogous results for the $s$ and $t$-channel boundary terms, we can express (4.18) as

$$
\begin{gathered}
\int d^{2} \sigma\left[-\frac{1}{2 \pi i}|\sigma|^{-u / 2-4}|\sigma-1|^{-s / 2-4}-\sum_{\substack{i, j \\
\alpha_{i}+\beta_{i}<0}} C_{i}(\sigma-1)^{\alpha_{i}-1}(\bar{\sigma}-1)^{\beta_{i}-1}\right. \\
\left.-\sum_{\substack{i, j \\
\alpha_{i}^{\prime}+\beta_{i}^{\prime}<0}} C_{i}^{\prime} \sigma^{\alpha_{i}^{\prime}-1} \bar{\sigma}^{\beta_{i}^{\prime}-1}-\sum_{\substack{i, j \\
\alpha_{i}^{\prime \prime}+\beta_{i}^{\prime \prime}<0}} C_{i}^{\prime \prime} \sigma^{-\alpha_{i}^{\prime \prime}-1} \bar{\sigma}^{-\beta_{i}^{\prime \prime}-1}\right] .
\end{gathered}
$$

The integral now runs over the full complex $\sigma$-plane, giving a finite expression for the amplitude since all the possible divergences near 0,1 and $\infty$ have been subtracted. In a related case, this form of the amplitude was used in [39].

\subsection{Comments}

We end this section with a few comments on our result.

1. One could ask whether the amplitudes defined via (4.18) or (4.28) agree with the ones defined by analytic continuation. Again string field theory can be invoked to prove the equality of these different procedures. Each tree level Feynman diagram constructed from string field theory is manifestly an analytic function of external momenta except for poles when an on-shell particle propagates in the intermediate state. Therefore if we begin in a region of the external momenta where there are no divergences in the world-sheet description and the string field theory and world-sheet descriptions

\footnotetext{
${ }^{9}$ For smaller size of $\widetilde{\mathcal{R}}_{u}$, both the bulk and the boundary integrals receive large contributions which cancel in the sum. Therefore if we take the size of $\widetilde{\mathcal{R}}_{u}$ to be very small, numerical errors will increase.
} 
coincide, and then analytically continue the result to the region where the direct world-sheet description gives divergent results, the result of analytic continuation will coincide with the one calculated from string field theory 1.e. eqs. (4.18) and (4.28).

2. Witten gave an operational procedure for performing the world-sheet integrals that coincides with the analytic continuation of the world-sheet description [23]. In spirit this corresponds to a different representation of the propagator (1.1). Instead of (1.3), we use (1.4). Using the relation between $\sigma \equiv y_{4}$ and $q$ described in (3.10), (3.17) and (3.20), we can convert the resulting contribution from $s, t$ and $u$ channel diagrams to appropriate contours in the complexified $\left(\sigma_{R}, \sigma_{I}\right)$ space where $\sigma_{R}$ and $\sigma_{I}$ are real and imaginary parts of $\sigma$. Since (1.4) gives the correct propagator for $L_{0}+\bar{L}_{0} \neq 0$, this prescription is guaranteed to coincide with eqs. (4.18) and (4.28).

\section{Higher point amplitudes}

We shall now generalize the procedure described above to higher point amplitudes of generic vertex operators for generic compactification. As in section 4 we shall decompose each propagator into two parts according to (1.3) and include the first part inside the square bracket as part of bulk integration while the second term inside the square bracket will provide a boundary term at $|q|=e^{-\Lambda}$. The main strategy will be to begin with the boundary terms that are implied by string field theory and then convert them to a general coordinate system in the moduli space. Our goal will be to arrive at an algorithm to compute the boundary terms in a general coordinate system without explicitly knowing the relation between the variables $q$ and the general coordinates used to parametrize the moduli space.

We shall begin by introducing a convenient way of labelling the propagators of a Feynman diagram. For a tree diagram, cutting a propagator divides the external states into two disconnected sets, with each set containing at least two external states. We associate to each propagator an index $s$ that specifies this division. Therefore $s$ can take $N$ possible values, where $N$ is the total number of ways in which the set of $(n+3)$ external states can be divided into two sets, with each set containing at least two external states. In a given Feynman diagram different propagators carry different labels, but two propagators from two different Feynman diagrams will carry the same label if cutting them leads to the same division of external particles into two sets. This can be illustrated using figure $1-$ the left internal propagator in the third diagram carries the same label as the propagator of the first diagram, while the right internal propagator in the third diagram carries the same label as the propagator of the second diagram. We shall denote by $q_{s}$ the variable $q$ used to represent the propagator carrying label $s$ as in (1.3).

Next we shall introduce some subspaces of the moduli space on which we have to evaluate the integrals. We denote by $\mathcal{C}^{(0)}$ the codimension 0 subspace of the moduli space of $(n+3)$-punctured sphere on which the bulk integration is performed. This includes contribution from the elementary $(n+3)$-point vertex, as well as all Feynman diagrams with propagators where from each propagator we include only the first term inside the 

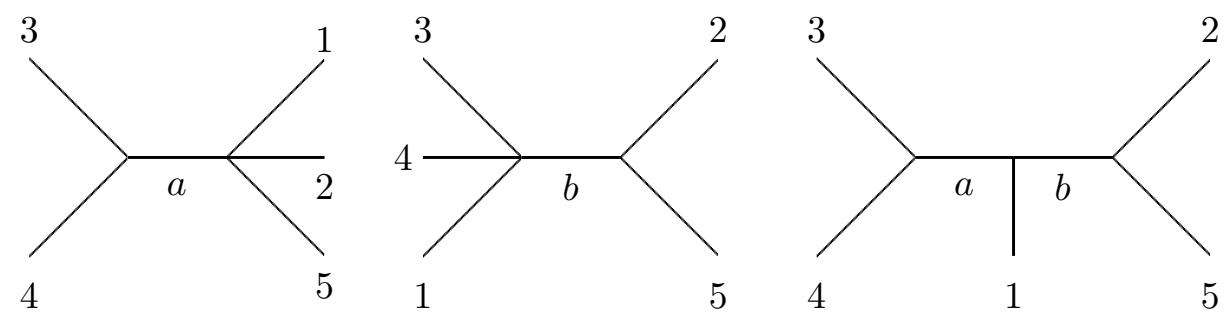

Figure 1. Examples of Feynman diagrams and the labelling of their boundaries in our convention.

square bracket in (1.3). In the variables $\left\{q_{s}\right\}$ this corresponds to restricting $\left|q_{s}\right| \geq e^{-\Lambda}$ for each $s$. We also denote by $-\mathcal{C}_{s}^{(1)}$ the boundary of $\mathcal{C}^{(0)}$ corresponding to setting, in all Feynman diagrams carrying a propagator with label $s$,

$$
\left|q_{s}\right|=e^{-\Lambda} \equiv \epsilon, \quad\left|q_{r}\right| \geq \epsilon \text { for } r \neq s .
$$

The - sign in front of $\mathcal{C}^{(1)}$ corresponds to the fact that the normal to $\mathcal{C}^{(1)}$ is taken to be along the direction of increasing $\left|q_{s}\right|$, pointing into $\mathcal{C}^{(0)}$. The region inside $\mathcal{C}^{(0)}$ corresponds to $\left|q_{s}\right|>\epsilon$. Even though for numerical evaluation it may be better to work with small $\epsilon$, all our formulae will be valid for finite $\epsilon \leq 1$.

We now denote by $\mathcal{C}_{s_{1} s_{2}}^{(2)}$ the codimension 2 subspace of the moduli space $\mathcal{C}^{(0)}$ associated with the intersection $\mathcal{C}_{s_{1}}^{(1)} \cap \mathcal{C}_{s_{2}}^{(1)}$. This corresponds to setting, in all Feynman diagrams that contain propagators carrying labels $s_{1}$ and $s_{2}$,

$$
\left|q_{s_{1}}\right|=\epsilon, \quad\left|q_{s_{2}}\right|=\epsilon, \quad\left|q_{s}\right| \geq \epsilon \text { for } s \neq s_{1}, s_{2} .
$$

We can regard $\mathcal{C}_{s_{1} s_{2}}^{(2)}$ as an oriented subspace that is anti-symmetric under the exchange of $s_{1}$ and $s_{2}$ since this exchanges their transverse coordinates $\left|q_{s_{1}}\right|$ and $\left|q_{s_{2}}\right|$. Generalizing this we define $\mathcal{C}_{s_{1} \cdots s_{k}}^{(k)}$ to be the codimension $k$ subspace obtained by setting

$$
\left|q_{s}\right|=\epsilon \quad \text { for } s=s_{1}, \cdots, s_{k}, \quad\left|q_{s}\right| \geq \epsilon \text { for } s \neq s_{1}, s_{2}, \cdots, s_{k},
$$

in all Feynman diagrams that contain propagators with labels $s_{1}, \cdots s_{k}$. It is easy to verify the relations

$$
C_{s_{1} \cdots s_{k}}^{(k)}=C_{s_{1}}^{(1)} \cap C_{s_{2}}^{(1)} \cap \cdots \cap C_{s_{k}}^{(1)},
$$

and

$$
\partial \mathcal{C}_{s_{1} \cdots s_{k}}^{(k)}=-\sum_{s} \mathcal{C}_{s_{1} \cdots s_{k} s}^{(k+1)}
$$

where the sum over $s$ runs over all labels for which $\mathcal{C}_{s_{1} \cdots s_{k} s}^{(k+1)}$ exists. As before, $\mathcal{C}_{s_{1} \cdots s_{k}}^{(k)}$ is antisymmetric under the exchange of the $s_{\ell}$ 's. The minus sign in (5.5) defines the orientation of the $\mathcal{C}^{(k+1)}$ 's for given orientation of the $\mathcal{C}^{(k)}$ 's.

Let $\mathcal{I}^{(0)}$ denote the bulk integrand that we need to integrate over the moduli space. The bulk contribution $\int_{\mathcal{C}}{ }^{(0)} \mathcal{I}^{(0)}$ comes from the sum of all Feynman diagrams, where in each Feynman diagram we take the product of the interaction vertices with the first terms inside the square bracket in (1.3) in all the propagators. Different Feynman diagrams cover 
different regions of $\mathcal{C}^{(0)}$. Now it follows from the definition of $\mathcal{C}_{s_{1} \cdots s_{k}}^{(k)}$ given above that if a Feynman diagram covers part of the region in $\mathcal{C}^{(0)}$ near $\mathcal{C}_{s_{1} \cdots s_{k}}^{(k)}$, then it must contain the propagators carrying labels $\left\{s_{1}, \cdots, s_{k}\right\}$ (and possibly other propagators), and we have $\left|q_{s}\right| \simeq e^{-\Lambda}$ for $s=s_{1}, \cdots, s_{k}$. Examination of the first term inside the square bracket in (1.3) shows that in a region of $\mathcal{C}^{(0)}$ near $\mathcal{C}_{s_{1} \cdots s_{k}}^{(k)}, \mathcal{I}^{(0)}$ has the form

$$
\mathcal{I}^{(0)}=\prod_{\ell=1}^{k}\left\{d q_{s_{\ell}} \wedge d \bar{q}_{s_{\ell}}\right\} \wedge d^{2(n-k)} m_{\left(s_{1}, \cdots, s_{k}\right)} \sum_{i} C_{i}\left(m_{\left(s_{1}, \cdots, s_{k}\right)}\right) \prod_{\ell=1}^{k} q_{s_{\ell}}^{-1+\gamma_{i}^{\left(s_{\ell}\right)}} \bar{q}_{s_{\ell}}^{-1+\delta_{i}^{\left(s_{\ell}\right)}},
$$

where $m_{\left(s_{1}, \cdots, s_{k}\right)}$ denote the coordinates of the $2(n-k)$ dimensional moduli space of the punctured spheres into which the original sphere degenerates when we set $q_{s_{1}}=\cdots=$ $q_{s_{k}}=0$. In a Feynman diagram of string field theory, these moduli come from the moduli integration appearing in the definition of the interaction vertices, as well as the parameters $q$ associated with the propagators other than those carrying labels $s_{1}, \cdots, s_{k}$. These, together with the phases of $q_{s_{1}}, \cdots, q_{s_{k}}$ form the coordinates of $\mathcal{C}_{s_{1} \cdots s_{k}}^{(k)} . C_{i}\left(m_{\left(s_{1}, \cdots, s_{k}\right)}\right)$ are some functions of these moduli given by the product of the vertices of the Feynman diagram and the first term inside the square bracket in (1.3) for the propagators other than the ones carrying labels $s_{1}, \cdots, s_{k} .\left(\gamma_{i}^{\left(s_{\ell}\right)}, \delta_{i}^{\left(s_{\ell}\right)}\right)$ are the $\left(L_{0}, \bar{L}_{0}\right)$ quantum numbers of states that can propagate in the propagator carrying the label $s_{\ell}$ for $1 \leq \ell \leq k$.

Using the second term in (1.3) we can also write down the expression for the integrand for the boundary term $\int_{\mathcal{C}_{s_{1} \cdots s_{k}}^{(k)}} \mathcal{I}_{s_{1} \cdots s_{k}}^{(k)}$, obtained by picking the second term inside the square bracket in (1.3) for the propagators carrying labels $s_{1}, \cdots, s_{k}$ and the first term inside the square bracket in (1.3) for all other propagators. The corresponding integrand $\mathcal{I}_{s_{1} \cdots s_{k}}^{(k)}$ is given by the $(2 n-k)$ form

$$
\begin{aligned}
\mathcal{I}_{s_{1} \cdots s_{k}}^{(k)}=(-1)^{k} d q_{s_{k}} \wedge \cdots \wedge d q_{s_{1}} \wedge d^{2(n-k)} m_{\left(s_{1}, \cdots, s_{k}\right)} & \\
& \sum_{i} C_{i}\left(m_{\left(s_{1}, \cdots, s_{k}\right)}\right) \prod_{\ell=1}^{k}\left\{\left(\delta_{i}^{\left(s_{\ell}\right)}\right)^{-1}\left(q_{s_{\ell}}\right)^{-1+\gamma_{i}^{\left(s_{\ell}\right)}}\left(\bar{q}_{s_{\ell}}\right)^{\delta_{i}^{\left(s_{\ell}\right)}}\right\} .
\end{aligned}
$$

We can now express the total contribution to the amplitude as:

$$
I=\sum_{k=0}^{n} \sum_{\substack{\left\{s_{1}, \cdots, s_{k}\right\} \\ s_{1}<s_{2}<\cdots<s_{k}}} \int_{\mathcal{C}_{s_{1} \cdots s_{k}}^{(k)}} \mathcal{I}_{s_{1} \cdots s_{k}}^{(k)}
$$

The sum runs over all inequivalent combinations $\left\{s_{1}, \cdots, s_{k}\right\}$ for which $\mathcal{C}_{s_{1} \cdots s_{k}}^{(k)}$ exists.

Using (5.6) with $k$ replaced by $k+j$, one also finds that near $\mathcal{C}_{s_{1} \cdots s_{k} r_{1} \cdots r_{j}}^{(k+j)} \subset \mathcal{C}_{s_{1} \cdots s_{k}}^{(k)}$,

$$
\begin{aligned}
& \mathcal{I}_{s_{1} \cdots s_{k}}^{(k)}=(-1)^{k} d q_{s_{k}} \wedge \cdots \wedge d q_{s_{1}} \wedge\left\{\prod_{a=1}^{j} d q_{r_{a}} \wedge d \bar{q}_{r_{a}}\right\} \wedge d^{2(n-k-j)} m_{\left(s_{1}, \cdots, s_{k}, r_{1}, \cdots, r_{j}\right)} \\
& \sum_{i} C_{i}\left(m_{\left(s_{1}, \cdots, s_{k}, r_{1}, \cdots, r_{j}\right)}\right) \prod_{\ell=1}^{k}\left\{\left(\delta_{i}^{\left(s_{\ell}\right)}\right)^{-1}\left(q_{s_{\ell}}\right)^{-1+\gamma_{i}^{\left(s_{\ell}\right)}}\left(\bar{q}_{s_{\ell}}\right)^{\delta_{i}^{\left(s_{\ell}\right)}}\right\} \\
& \left\{\prod_{b=1}^{j}\left(q_{r_{b}}\right)^{-1+\gamma_{i}^{\left(r_{b}\right)}}\left(\bar{q}_{r_{b}}\right)^{-1+\delta_{i}^{\left(r_{b}\right)}}\right\} \text {. }
\end{aligned}
$$


Note that the $C_{i}$ 's in (5.7) and (5.9) are not the same, but the latter are obtained by expanding the former near $\mathcal{C}_{s_{1} \cdots s_{k} r_{1} \cdots r_{j}}^{(k+j)}$. It is easy to verify from (5.7), and (5.9) with $j=1$, $k$ replaced by $(k-1)$, that in the neighborhood of $\mathcal{C}_{s_{1} \cdots s_{k}}^{(k)}$ :

$$
d \mathcal{I}_{s_{1} \cdots s_{k}}^{(k)}=\mathcal{I}_{s_{1} \cdots s_{k-1}}^{(k-1)}-\mathcal{I}_{s_{1} \cdots s_{k-2} s_{k}}^{(k-1)}+\cdots+(-1)^{k-1} \mathcal{I}_{s_{2} \cdots s_{k}}^{(k-1)}
$$

Note that (5.8) and (5.10) are written in the coordinate free notation. Therefore we can use these to give an expression for the amplitude in an arbitrary coordinate system in the moduli space without referring to the coordinates $q_{s}, \bar{q}_{s}$. However we need to first check if these equations fix the amplitude uniquely. First we see from (5.10) that for non-integer $\gamma_{i}^{(s)}, \delta_{i}^{(s)}$, there is an ambiguity in determining $\mathcal{I}^{(k)}$ for given $\mathcal{I}^{(k-1)}$ of the form:

$$
\mathcal{I}_{s_{1} \cdots s_{k}}^{(k)} \rightarrow \mathcal{I}_{s_{1} \cdots s_{k}}^{(k)}+d \mathcal{J}_{s_{1} \cdots s_{k}}^{(k)},
$$

where $\mathcal{J}_{s_{1} \cdots s_{k}}^{(k)}$ is an $(2 n-k-1)$ form defined near $\mathcal{C}_{s_{1} \cdots s_{k}}^{(k)}$. Due to (5.10) with $k$ replaced by $k+1$, this requires us to change $\mathcal{I}_{s_{1} \cdots s_{k} s_{k+1}}^{(k+1)}$ as

$$
\mathcal{I}_{s_{1} \cdots s_{k} s_{k+1}}^{(k+1)} \rightarrow \mathcal{I}_{s_{1} \cdots s_{k} s_{k+1}}^{(k+1)}+\sum_{\ell=1}^{k+1} \mathcal{J}_{s_{1} \cdots s_{\ell-1} s_{\ell+1} s_{k+1}}^{(k)}(-1)^{k+1-\ell} .
$$

Therefore the net ambiguity in the whole set $\left\{\mathcal{I}_{s_{1} \cdots s_{k}}^{(k)}\right\}$ for all $k$ and $\left\{s_{1}, \cdots, s_{k}\right\}$ satisfying (5.10) takes the form

$$
\Delta \mathcal{I}_{s_{1} \cdots s_{k}}^{(k)}=d \mathcal{J}_{s_{1} \cdots s_{k}}^{(k)}+\sum_{\ell=1}^{k} \mathcal{J}_{s_{1} \cdots s_{\ell-1} s_{\ell+1} \cdots s_{k}}^{(k-1)}(-1)^{k-\ell} .
$$

We shall now compute its effect on the total integral $I$ defined in (5.8). We get

$$
\Delta I=\sum_{k=0}^{n} \sum_{\substack{\left\{s_{1}, \cdots, s_{k}\right\} \\ s_{1}<s_{2}<\cdots<s_{k}}} \int_{\mathcal{C}_{s_{1} \cdots s_{k}}^{(k)}}\left\{d \mathcal{J}_{s_{1} \cdots s_{k}}^{(k)}+\sum_{\ell=1}^{k} \mathcal{J}_{s_{1} \cdots s_{\ell-1} s_{\ell+1} \cdots s_{k}}^{(k-1)}(-1)^{k-\ell}\right\} .
$$

Using (5.5) we get

$$
\int_{\mathcal{C}_{s_{1} \cdots s_{k}}^{(k)}} d \mathcal{J}_{s_{1} \cdots s_{k}}^{(k)}=\int_{\partial \mathcal{C}_{s_{1} \cdots s_{k}}^{(k)}} \mathcal{J}_{s_{1} \cdots s_{k}}^{(k)}=-\sum_{s \neq s_{1}, \cdots, s_{k}} \int_{\mathcal{C}_{s_{1} \cdots s_{k} s}^{(k+1)}} \mathcal{J}_{s_{1} \cdots s_{k}}^{(k)} .
$$

On the other hand, replacing the summation variable $k$ by $k+1$ we can express the second term in (5.14) as

$$
\begin{aligned}
& \sum_{k=0}^{n} \sum_{\substack{\left\{s_{1}, \cdots, s_{k+1}\right\} \\
s_{1}<s_{2}<\cdots<s_{k+1}}} \sum_{\ell=1}^{k+1} \int_{\mathcal{C}_{s_{1} \cdots s_{k} s_{k+1}}^{(k+1)}} \mathcal{J}_{s_{1} \cdots s_{\ell-1} s_{\ell+1} \cdots s_{k+1}}^{(k)}(-1)^{k+1-\ell} \\
= & \sum_{k=0}^{n} \sum_{\substack{\left\{s_{1}, \cdots, s_{k}\right\} \\
s_{1}<s_{2}<\cdots<s_{k}}} \sum_{s \neq s_{1}, \cdots, s_{k}} \int_{\mathcal{C}_{s_{1} \cdots s_{k} s}^{(k+1)}} \mathcal{J}_{s_{1} \cdots s_{k}}^{(k)}
\end{aligned}
$$

where in the last step we have rearranged the sum by relabelling $\left\{s_{1}, \cdots, s_{\ell-1}, s_{\ell+1}, \cdots, s_{k+1}\right\}$ as $\left\{s_{1}, \cdots, s_{k}\right\}$ and $s_{\ell}$ as $s$, and used the antisymmetry of $\mathcal{C}^{(k+1)}$ to write $(-1)^{k+1-\ell} \mathcal{C}_{s_{1} \cdots s_{k} s_{k+1}}^{(k+1)}$ 
as $\mathcal{C}_{s_{1} \cdots s_{k} s}^{(k+1)}$. Substituting (5.15) and (5.16) into (5.14) we see that the two terms cancel and we get

$$
\Delta I=0 .
$$

Therefore the ambiguity (5.13) in the determination of $\mathcal{I}^{(k)}$ by solving (5.10) does not affect the integral $I$ given in (5.8). This means that we can solve (5.10) to determine the boundary corrections directly by examining the form of the bulk integrand in any given coordinate system, without having to know the relation between the chosen coordinates and the $q_{s}$ 's near the boundaries. As already emphasized before, this requires us to work with generic external momenta so that the exponents $\gamma_{i}^{\left(s_{\ell}\right)}, \delta_{i}^{\left(s_{\ell}\right)}$ are not integers. Otherwise the determination of $\mathcal{I}_{s}^{(1)}$ could have an additive ambiguity proportional to $q_{s}^{-1} d q_{s} d^{2(n-1)} m_{(s)} C\left(m_{(s)}\right)$ that is not an exact differential $d \mathcal{J}_{s}^{(1)}$. Similar ambiguities could be present in the other $\mathcal{I}_{s_{1} \cdots s_{k}}^{(k)}$ 's as well.

Now the expression for the amplitude given in (5.8) depends not only on the differential forms $\mathcal{I}_{s_{1} \cdots s_{k}}^{(k)}$ but also on the boundaries $\mathcal{C}_{s_{1} \cdots s_{k}}^{(k)}$ on which these forms are to be integrated. Since these are given by $\left|q_{s_{1}}\right|=\cdots=\left|q_{s_{k}}\right|=\epsilon$, the shapes of these boundaries in a generic coordinate system depend on the relation between the special coordinates $\left\{q_{s_{\ell}}\right\}$ induced from string field theory and the coordinates we are using (which could be taken to be the positions $\sigma_{1}, \cdots, \sigma_{n}$ of $n$ of the vertex operators keeping three of them at fixed positions). We shall now demonstrate that (5.8) is actually invariant under arbitrary deformations of $\mathcal{C}_{s}^{(1)}$ 's and consequent deformation of the $\mathcal{C}_{s_{1} \cdots s_{k}}^{(k)}$ 's following from (5.4). For this instead of considering the most general deformation let us consider the infinitesimal deformation $\delta_{s}$ that moves the boundary $\mathcal{C}_{s}^{(1)}$ to a new position $\mathcal{C}_{s}^{(1) \prime}$. Clearly a generic infinitesimal deformation can be regarded as a linear combination of the $\delta_{s}$ 's. Under such a deformation we have two kinds of effects:

1. The manifold $\mathcal{C}_{s_{1} \cdots s_{k} s}^{(k+1)}$, being a subspace of $\mathcal{C}_{s}^{(1)}$, gets shifted to a new manifold $\mathcal{C}_{s_{1} \cdots s_{k} s}^{(k+1)}$.

2. The manifold $\mathcal{C}_{s_{1} \cdots s_{k}}^{(k)}$ for $s \neq s_{1}, \cdots s_{k}$, having a boundary $-\mathcal{C}_{s_{1} \cdots s_{k} s}^{(k)}$ inside $\mathcal{C}_{s}^{(1)}$, gets extended by an amount $\delta_{s} \mathcal{C}_{s_{1} \cdots s_{k}}^{(k)}$.

Let us consider the subspace $\delta_{s} \mathcal{C}_{s_{1} \cdots s_{k}}^{(k)}$. Its boundaries are given as follows. $-\mathcal{C}_{s_{1} \cdots s_{k} s}^{(k+1)}$ was a boundary of the original $\mathcal{C}_{s_{1} \cdots s_{k}}^{(k)}$ which has now moved to $-\mathcal{C}_{s_{1} \cdots s_{k} s}^{\prime(k+1)}$. Therefore both $-\mathcal{C}_{s_{1} \cdots s_{k} s}^{\prime(k+1)}$ and $\mathcal{C}_{s_{1} \cdots s_{k} s}^{(k+1)}$ form boundaries of $\delta_{s} \mathcal{C}_{s_{1} \cdots s_{k}}^{(k)}$. Besides this $\delta_{s} \mathcal{C}_{s_{1} \cdots s_{k}}^{(k)}$ share the extensions of the boundaries $-\mathcal{C}_{s_{1} \cdots s_{k} r}^{(k)}$ for $r \neq s$ of $\mathcal{C}_{s_{1} \cdots s_{k}}^{(k)}$ which have been extended by $\delta_{s} \mathcal{C}_{s_{1} \cdots s_{k} r}^{(k)}$. Therefore we have the relation

$$
\partial \delta_{s} \mathcal{C}_{s_{1} \cdots s_{k}}^{(k)}=-\mathcal{C}_{s_{1} \cdots s_{k} s}^{(k+1)}+\mathcal{C}_{s_{1} \cdots s_{k} s}^{(k+1)}-\sum_{r \neq s, s_{1}, \cdots, s_{k}} \delta_{s} \mathcal{C}_{s_{1} \cdots s_{k} r}^{(k+1)}
$$

The net change in $I$ under such a deformation is given by

$$
\begin{aligned}
\delta_{s} I= & \sum_{k=0}^{n} \sum_{\substack{\left\{s_{1}, \cdots, s_{k}\right\} \\
s_{1}<s_{2}<\cdots<s_{k}, s_{1}, \cdots, s_{k} \neq s}} \int_{\delta_{s} \mathcal{C}_{s_{1} \cdots s_{k}}^{(k)}} \mathcal{I}_{s_{1} \cdots s_{k}}^{(k)} \\
& +\sum_{k=0}^{n} \sum_{\substack{\left\{s_{1}, \cdots, s_{k}\right\} \\
s_{1}<s_{2}<\cdots<s_{k}, s_{1}, \cdots, s_{k} \neq s}}\left[\int_{\mathcal{C}_{s_{1} \cdots s_{k} s}^{(k)}} \mathcal{I}_{s_{1} \cdots s_{k} s}^{(k+1)}-\int_{\mathcal{C}_{s_{1} \cdots s_{k} s}^{(k)}} \mathcal{I}_{s_{1} \cdots s_{k} s}^{(k+1)}\right] .
\end{aligned}
$$


Using (5.18) we can express (5.19) as

$$
\begin{aligned}
\delta_{s} I= & \sum_{k=0}^{n} \sum_{\substack{\left\{s_{1}, \cdots, s_{k}\right\} \\
s_{1}<s_{2}<\cdots<s_{k}, s_{1}, \cdots, s_{k} \neq s}} \int_{\delta_{s} \mathcal{C}_{s_{1} \cdots s_{k}}^{(k)}} \mathcal{I}_{s_{1} \cdots s_{k}}^{(k)} \\
& -\sum_{k=0}^{n} \sum_{\substack{\left\{s_{1}, \cdots, s_{k}\right\} \\
s_{1}<s_{2}<\cdots<s_{k}, s_{1}, \cdots, s_{k} \neq s}}\left[\int_{\partial \delta_{s} \mathcal{C}_{s_{1} \cdots s_{k}}^{(k)}} \mathcal{I}_{s_{1} \cdots s_{k} s}^{(k+1)}+\sum_{r \neq s, s_{1}, \cdots, s_{k}} \int_{\delta_{s_{s}} \mathcal{C}_{s_{1} \cdots s_{k} r}^{(k+1)}} \mathcal{I}_{s_{1} \cdots s_{k} s}^{(k+1)}\right] .
\end{aligned}
$$

Using (5.10) the first term inside the square bracket in the second line can be manipulated as

$$
\begin{aligned}
\int_{\partial \delta_{s} \mathcal{C}_{s_{1} \cdots s_{k}}^{(k)}} \mathcal{I}_{s_{1} \cdots s_{k} s}^{(k+1)} & =\int_{\delta_{s} \mathcal{C}_{s_{1} \cdots s_{k}}^{(k)}} d \mathcal{I}_{s_{1} \cdots s_{k} s}^{(k+1)} \\
& =\int_{\delta_{s} \mathcal{C}_{s_{1} \cdots s_{k}}^{(k)}}\left[\mathcal{I}_{s_{1} \cdots s_{k}}^{(k)}+\sum_{\ell=1}^{k}(-1)^{k+1-\ell} \mathcal{I}_{s_{1} \cdots s_{\ell-1} s_{\ell+1} \cdots s_{k} s}^{(k)}\right] .
\end{aligned}
$$

Substituting this into (5.20) we get

$$
\begin{aligned}
\delta_{s} I= & \sum_{k=0}^{n} \sum_{\substack{\left\{s_{1}, \cdots, s_{k}\right\} \\
s_{1}<s_{2}<\cdots<s_{k}, s_{1}, \cdots, s_{k} \neq s}} \int_{\delta_{s} \mathcal{C}_{s_{1} \cdots s_{k}}^{(k)}} \sum_{\ell=1}^{k}(-1)^{k-\ell} \mathcal{I}_{s_{1} \cdots s_{\ell-1} s_{\ell+1} \cdots s_{k} s}^{(k)} \sum_{\substack{\left\{s_{1}, \cdots, s_{k}\right\}\\
}} \sum_{\substack{r, s_{1}, \cdots, s_{k} \\
s_{1}<s_{2}<\cdots<s_{k}, s_{1}, \cdots, s_{k} \neq s}} \int_{\delta_{s} \mathcal{C}_{s_{1} \cdots s_{k} r}^{(k+1)}} \mathcal{I}_{s_{1} \cdots s_{k} s}^{(k+1)} .
\end{aligned}
$$

By making a $k \rightarrow(k+1)$ shift in the first term and relabelling $s_{\ell}$ as $r,\left\{s_{1}, \cdots s_{\ell-1}, s_{\ell+1}, \cdots\right.$, $\left.s_{k+1}\right\}$ as $\left\{s_{1}, \cdots, s_{k}\right\}$, we see that the two terms in (5.22) cancel and we have

$$
\delta_{s} I=0 .
$$

This shows that (5.8) is invariant under the deformations of the subspaces $\mathcal{C}_{s}^{(1)}$.

Using these results we arrive at the following algorithm for evaluating an $(n+3)$-point amplitude:

1. We can take three of the vertex operators at fixed locations $y_{n+1}, y_{n+2}$ and $y_{n+3}$ and take the locations $\sigma_{1}, \cdots \sigma_{n}$ of the other vertex operators as coordinates of the moduli space.

2. We now list all possible ways of dividing the set $1, \cdots,(n+3)$ into two subsets, with each subset containing at least two particles. Associated with each such decomposition, labelled by $s$, there is a possible degeneration in which the original sphere degenerates into two spheres, each carrying external punctures belonging to one of the two subsets. For every such degeneration, we can construct, in terms of original moduli $\left\{\sigma_{i}\right\}$, a new complex parameter $u_{s}$ that vanishes at the degeneration and a set of $(n-1)$ complex moduli $m_{(s)}$ labelling the moduli of the spheres left after degeneration. The change of variables from $\left\{\sigma_{i}\right\}$ to $u_{s}, m_{(s)}$ may not be globally defined over the whole moduli space spanned by $m_{(s)}$ and we typically will have to use different $u_{s}$ in different patches. 
3. Multiple degenerations will correspond to several of the $u_{s}$ 's vanishing simultaneously. Near a degeneration where $u_{s_{1}}, \cdots, u_{s_{k}}$ vanish, we can construct, in terms of the $\left\{\sigma_{i}\right\}$ 's, a set of $(n-k)$ complex coordinates $m_{\left(s_{1}, \cdots, s_{k}\right)}$ which remain finite at the degeneration, labelling the moduli of the spheres into which the original sphere degenerates. We can use $u_{s_{1}}, \cdots, u_{s_{k}}$ and $m_{\left(s_{1}, \cdots, s_{k}\right)}$ to parametrize the moduli space near such degenerations.

4. We now denote by $\mathcal{D}_{s}$ an open tubular neighborhood of $u_{s}=0$ and define $\mathcal{C}^{(0)}$ to be the region of the moduli space that excludes $\mathcal{D}_{s}$ for all $s$. We also denote by $\mathcal{C}_{s}^{(1)}$ the intersection $\partial \mathcal{D}_{s} \cap \mathcal{C}^{(0)}$, forming a component of the boundary of $\mathcal{C}^{(0)}$, and by $\mathcal{C}_{s_{1} \cdots s_{k}}^{(k)}$ the codimension $k$ intersection $\mathcal{C}_{s_{1}}^{(1)} \cap \mathcal{C}_{s_{2}}^{(1)} \cap \cdots \cap \mathcal{C}_{s_{k}}^{(1)}$.

5. Once we know the relation between the coordinates $\sigma_{1}, \cdots, \sigma_{n}$ and $\left\{u_{s}\right\}$ and $\left\{m_{\left(s_{1}, \cdots, s_{k}\right)}\right\}$, we can determine $\mathcal{I}_{s_{1} \cdots s_{k}}^{(k)}$ as follows. $\mathcal{I}^{(0)}$ is the integrand of the original amplitude given by correlation functions of vertex operators in the CFT. $\mathcal{I}^{(0)}$ is originally expressed in terms of $\sigma_{i}$ 's, but given the known relation between the $\sigma_{i}$ 's and $u_{s}, m_{(s)}$ we can expand it near $\mathcal{C}_{s}^{(1)}$ in a power series in $u_{s}$ of the form:

$$
\mathcal{I}^{(0)}=d u_{s} \wedge d \bar{u}_{s} \wedge d^{2(n-1)} m_{(s)} \sum_{i} K_{i}^{(s)}\left(m_{(s)}\right) u_{s}^{-1+\alpha_{i}^{(s)}} \bar{u}_{s}^{-1+\beta_{i}^{(s)}}
$$

for appropriate constants $\alpha_{i}^{(s)}, \beta_{i}^{(s)}$ and functions $K_{i}^{(s)}\left(m_{(s)}\right)$. Given this we can find $\mathcal{I}_{s}^{(1)}$ satisfying (5.10) as

$$
\mathcal{I}_{s}^{(1)}=-d u_{s} \wedge d^{2(n-1)} m_{(s)} \sum_{i}\left(\beta_{i}^{(s)}\right)^{-1} K_{i}^{(s)}\left(m_{(s)}\right) u_{s}^{-1+\alpha_{i}^{(s)}} \bar{u}_{s}^{\beta_{i}^{(s)}},
$$

up to addition of exact forms which, as we have argued before, does not affect the final result. We now expand $\mathcal{I}_{s_{1}}^{(1)}$ and $\mathcal{I}_{s_{2}}^{(2)}$ near $\mathcal{C}_{s_{1} s_{2}}^{(2)}$ as a power series in $u_{s_{1}}, u_{s_{2}}$ and their complex conjugates, with the coefficients of expansion given as functions of $m_{\left(s_{1}, s_{2}\right)}$. This can be done by using the known relations between the coordinate systems $\left\{u_{s_{1}}, \bar{u}_{s_{1}}, m_{\left(s_{1}\right)}\right\},\left\{u_{s_{1}}, \bar{u}_{s_{1}}, u_{s_{2}}, \bar{u}_{s_{2}}, m_{\left(s_{1}, s_{2}\right)}\right\}$ and $\left\{u_{s_{2}}, \bar{u}_{s_{2}}, m_{\left(s_{2}\right)}\right\}$, each of which can be related to the original coordinates $\left\{\sigma_{1}, \cdots, \sigma_{n}\right\}$. Given $\mathcal{I}_{s_{1}}^{(1)}$ and $\mathcal{I}_{s_{2}}^{(1)}$, both expressed in the same coordinate system, we now look for $\mathcal{I}_{s_{1} s_{2}}^{(2)}$ satisfying

$$
d \mathcal{I}_{s_{1} s_{2}}^{(2)}=\mathcal{I}_{s_{1}}^{(1)}-\mathcal{I}_{s_{2}}^{(1)}
$$

with $\mathcal{I}_{s_{1} s_{2}}^{(2)}$ having an expansion in powers of $u_{s_{1}}, u_{s_{2}}$ and their complex conjugates, with the coefficients of expansion given as functions of the remaining moduli $m_{\left(s_{1}, s_{2}\right)}$. The existence of such solutions is guaranteed by our general argument. Proceeding this way we can construct all the $\mathcal{I}_{s_{1} \cdots s_{k}}^{(k)}$ 's using (5.10).

6. Once all the $\mathcal{I}_{s_{1} \cdots s_{k}}^{(k)}$ 's and $\mathcal{C}_{s_{1} \cdots s_{k}}^{(k)}$ 's have been constructed, we can compute the amplitude using (5.8). 
7. As already mentioned, the relation between the coordinate system $\left\{u_{s}\right\},\left\{m_{\left(s_{1}, \cdots, s_{k}\right)}\right\}$ and the global coordinates $\left\{\sigma_{1}, \cdots, \sigma_{n}\right\}$ may differ in different patches of the moduli space. In particular the natural choice of the coordinates $u_{s_{1}}, \cdots, u_{s_{k}}$ near $\mathcal{C}_{s_{1} \cdots s_{k}}^{(k)}$ may differ from the natural choice of the $u_{s}$ 's near the $\mathcal{C}^{(k-1)}$ 's that are used to describe the $\mathcal{I}^{(k-1)}$ 's. Since the relations between these coordinates and $\left\{\sigma_{i}\right\}$ 's and the old coordinates and $\left\{\sigma_{i}\right\}$ 's are known, we can always express the $\mathcal{I}^{(k-1)}$ 's determined in the previous step in the new coordinate system and proceed as above. Example of such changes in coordinate system will be described in appendix A, where we have described possible choice of coordinates $\left\{u_{s}\right\}, m_{\left(s_{1}, \cdots, s_{k}\right)}$ on a five punctured sphere.

\section{Amplitudes in superstring theory}

In superstring theory the computation of amplitudes suffers from divergences similar to the ones described above for bosonic string theory - appearing from propagation of internal states carrying negative $L_{0}+\bar{L}_{0}$ eigenvalue. However there is another source of divergences in superstring theory - from the wrong choice of PCOs. Consider for example a pair of integrated -1 picture vertex operators $e^{-\phi} \psi^{\mu} \bar{\partial} X^{\nu}(z, \bar{z})$ and $e^{-\phi} \psi^{\rho} \bar{\partial} X^{\sigma}(w, \bar{w})$. The rule that follows from superstring field theory is that when we bring them close, we must also bring a PCO close to them so that their product generates a -1 picture state. This can be achieved e.g. by converting the second vertex operator to a zero picture vertex operator $\partial X^{\rho} \bar{\partial} X^{\sigma}(w, \bar{w})$. The leading singularity in the collision of the two vertex operators now is proportional to $(\bar{z}-\bar{w})^{-2} e^{-\phi} \psi^{\mu} \partial X^{\rho} \eta^{\nu \sigma}$. If instead we had taken the product of the two -1 picture vertex operators, then we would have gotten a stronger leading singularity proportional to $(z-w)^{-2}(\bar{z}-\bar{w})^{-2} e^{-2 \phi} \eta^{\mu \rho} \eta^{\nu \sigma}$. A similar situation would arise if we had converted both vertex operators to zero picture before bringing them close to each other.

Now often it is convenient to take the PCO's to coincide with some vertex operators before computing the correlation function and use the same arrangement everywhere in the moduli space. According to the discussion in the preceding paragraph this would give the wrong integrand $\mathcal{I}^{(0)}$ near many degenerations, e.g. when two or more zero picture vertex operators come together their product would carry a net picture number 0 instead of -1 . Therefore this would be the wrong starting point for implementing the procedure described in section 5. However one can show that the error that one makes by taking the wrong choice of PCO locations adds to $\mathcal{I}^{(0)}$ an exact differential in $\mathcal{C}^{(0)}$. We shall now argue that the addition of such exact differentials does not affect the amplitude $I$ defined in (5.8). For this let us suppose that we change $\mathcal{I}^{(0)}$ to $\mathcal{I}^{(0)}+d \mathcal{L}^{(1)}$ where $\mathcal{L}^{(1)}$ is a globally defined $2 n-1$ form on $\mathcal{C}^{(0)}$. Then the equation $d \mathcal{I}_{s}^{(1)}=\mathcal{I}^{(0)}$ may be solved by shifting $\mathcal{I}_{s}^{(1)}$ to $\mathcal{I}_{s}^{(1)}+\mathcal{L}^{(1)}$. The next equation

$$
d \mathcal{I}_{s_{1} s_{2}}^{(2)}=\mathcal{I}_{s_{1}}^{(1)}-\mathcal{I}_{s_{2}}^{(1)}
$$

is not affected by this change since the extra term $\mathcal{L}^{(1)}$ in $\mathcal{I}_{s_{1}}^{(1)}$ and $\mathcal{I}_{s_{2}}^{(1)}$ cancel. Therefore $\mathcal{I}_{s_{1} \cdots s_{k}}^{(k)}$ for $k \geq 2$ remain unchanged. 
We can now study the effect of the change in $\mathcal{I}^{(0)}$ and $\mathcal{I}^{(1)}$ on $I$ defined in (5.8). We have

$$
\int_{\mathcal{C}^{(0)}} \mathcal{I}^{(0)} \rightarrow \int_{\mathcal{C}^{(0)}} \mathcal{I}^{(0)}+\int_{\mathcal{C}^{(0)}} d \mathcal{L}^{(1)}=\int_{\mathcal{C}^{(0)}} \mathcal{I}^{(0)}+\int_{\partial \mathcal{C}^{(0)}} \mathcal{L}^{(1)}=\int_{\mathcal{C}^{(0)}} \mathcal{I}^{(0)}-\sum_{s} \int_{\mathcal{C}_{s}^{(1)}} \mathcal{L}^{(1)}
$$

and

$$
\sum_{s} \int_{\mathcal{C}_{s}^{(1)}} \mathcal{I}_{s}^{(1)} \rightarrow \sum_{s} \int_{\mathcal{C}_{s}^{(1)}} \mathcal{I}_{s}^{(1)}+\sum_{s} \int_{\mathcal{C}_{s}^{(1)}} \mathcal{L}^{(1)}
$$

Therefore the net change in the sum of the left hand sides of (6.2) and (6.3) vanishes, showing that the expression corrects itself even if we evaluate the integrand with the wrong choice of PCO locations. However this procedure works only for generic external momenta for which the exponents $\gamma_{i}^{(s)}, \delta_{i}^{(s)}$ appearing in (5.6) are not integers.

\section{Mass shift under marginal deformation}

We shall now consider a different situation in tree level string theory where short distance divergences appear in the world-sheet of the string, and show how string field theory removes the divergence. The case that we shall describe involves marginal deformation of the matter CFT that is used in describing the target space geometry. Since the deformed background is also a conformal field theory, one can formulate string theory around the deformed background as well. The spectrum and S-matrix of the new theory is in principle computable from the data in the original CFT before the deformation using conformal perturbation theory. This however requires ultraviolet regularization on the world-sheet, since the naive computation requires integrating correlation functions of marginal operators and other operators over the locations of the marginal operators and they diverge when the marginal operators collide with each other or with other operators. Our goal will be to show how in string field theory we can carry out the computation without encountering any divergence.

Although the procedure we shall describe is valid for any marginal deformation, in order to get concrete results we shall focus on a particular class of examples where the target space includes a compact circle and the marginal deformation corresponds to deforming the radius of the compact circle. We shall denote the world-sheet scalar field corresponding to the compact circle by $Y$. In string field theory the marginal deformation that changes the radius of the circle can be represented as the effect of switching on a background string field solving the classical equations of motion. We shall use string field theory to compute the shift in the masses of various states under this deformation to second order in the deformation parameter and compare the result to known results. In doing this we shall make use of the general strategy described in [35] for solving classical equations of motion of string field theory. During this analysis we shall see that while in the intermediate stages of calculation the results depend on the choice of local coordinates used to define the theory, the final result is independent of this choice. 


\subsection{Bosonic string theory}

The equation of motion of bosonic string field theory is given by (2.19):

$$
Q_{B}|\Psi\rangle+\sum_{N=2}^{\infty} \frac{1}{N !}\left[\Psi^{N}\right]=0 .
$$

We shall solve this equation in a power series in the parameter $\mu$ :

$$
\left|\Psi^{\mathrm{cl}}\right\rangle=\sum_{n \geq 1} \mu^{n}\left|\Psi_{n}^{\mathrm{cl}}\right\rangle
$$

by starting with a solution

$$
\mu\left|\Psi_{1}^{\mathrm{cl}}\right\rangle=\mu c_{1} \bar{c}_{1} V(0)|0\rangle, \quad V=\partial Y \bar{\partial} Y .
$$

It is clear that this solves (7.1) to order $\mu$ since $\left|\Psi_{1}^{\mathrm{cl}}\right\rangle$ is annihilated by $Q_{B}$. The second order correction $\mu^{2}\left|\Psi_{2}^{\text {cl }}\right\rangle$ to the solution can be expressed as [35]

$$
\left|\Psi_{2}^{\mathrm{cl}}\right\rangle=-\frac{1}{2} \frac{b_{0}^{+}}{L_{0}^{+}}(1-P)\left[\Psi_{1}^{\mathrm{cl}} \Psi_{1}^{\mathrm{cl}}\right]+\left|\psi_{2}^{\mathrm{cl}}\right\rangle,
$$

where $P$ is the projection operator into the $L_{0}^{+}=0$ states and $\left|\psi_{2}^{\mathrm{cl}}\right\rangle \in P \mathcal{H}$ satisfies:

$$
Q_{B}\left|\psi_{2}^{\mathrm{cl}}\right\rangle=-\frac{1}{2} P\left[\Psi_{1}^{\mathrm{cl}} \Psi_{1}^{\mathrm{cl}}\right]
$$

It is easy to see however that for the choice given in (7.3), the right hand side of the above equation vanishes. For this we can take the inner product of this with $\langle\phi| c_{0}^{-}$for any ghost number 2 state $|\phi\rangle$ in $\mathcal{H}$ with $L_{0}^{+}=0$ and identify this with a 3-point function $\left\{\phi \Psi_{1}^{\mathrm{cl}} \Psi_{1}^{\mathrm{cl}}\right\}$ using (2.18). Since (7.3) is invariant under $Y \rightarrow-Y$, we can restrict to states invariant under this transformation. Also we can ignore states involving excitations in the parts of the matter CFT other than the one involving $Y$, since one point functions of the corresponding vertex operators vanish. The relevant states $|\phi\rangle$ are

$$
c_{1} c_{-1}|0\rangle, \quad \bar{c}_{1} \bar{c}_{-1}|0\rangle, \quad c_{1} \bar{c}_{1} V(0)|0\rangle .
$$

For the first two states in (7.6), $\left\{\phi \Psi_{1}^{\mathrm{cl}} \Psi_{1}^{\mathrm{cl}}\right\}$ vanishes since in the three point function the total ghost number has to add to 3 separately in the left and the right sector. For the last state in (7.6), $\left\{\phi \Psi_{1}^{\mathrm{cl}} \Psi_{1}^{\mathrm{cl}}\right\}$ vanishes since the three point function of three $\partial Y \bar{\partial} Y$ 's vanishes due to separate $Y \rightarrow-Y$ symmetry in the left and the right sector. ${ }^{10}$ Therefore we can take

$$
\left|\psi_{2}^{\mathrm{cl}}\right\rangle=0
$$

Since the states listed in (7.6) are all BRST invariant, we could include an arbitrary linear combination of these states in the definition of $\left|\psi_{2}^{\text {cl }}\right\rangle$. This will correspond to a redefinition of $\mu$ or the string coupling constant, or a gauge transformation.

\footnotetext{
${ }^{10}$ For a general marginal deformation, vanishing of the three point function is a requirement for exact marginality.
} 
Even though we shall use the solution to order $\mu^{2}$, it is instructive to examine how the solution can be extended to order $\mu^{3}$. We can solve (7.1) by taking [35]

$$
\left|\Psi_{3}^{\mathrm{cl}}\right\rangle=-\frac{b_{0}^{+}}{L_{0}^{+}}(1-P)\left(\frac{1}{6}\left[\Psi_{1}^{\mathrm{cl}} \Psi_{1}^{\mathrm{cl}} \Psi_{1}^{\mathrm{cl}}\right]+\left[\Psi_{1}^{\mathrm{cl}} \Psi_{2}^{\mathrm{cl}}\right]\right)+\left|\psi_{3}^{\mathrm{cl}}\right\rangle
$$

where $\left|\psi_{3}^{\mathrm{cl}}\right\rangle \in P \mathcal{H}$ satisfies

$$
Q_{B}\left|\psi_{3}^{\mathrm{cl}}\right\rangle=-\frac{1}{6} P\left[\Psi_{1}^{\mathrm{cl}} \Psi_{1}^{\mathrm{cl}} \Psi_{1}^{\mathrm{cl}}\right]-P\left[\Psi_{1}^{\mathrm{cl}} \Psi_{2}^{\mathrm{cl}}\right]
$$

Possible obstruction to extending the solution to order $\mu^{3}$ can arise from the failure to solve (7.9). Using (7.4) and (7.7), we can express this as

$$
Q_{B}\left|\psi_{3}^{\mathrm{cl}}\right\rangle=-P\left(\frac{1}{6}\left[\Psi_{1}^{\mathrm{cl}} \Psi_{1}^{\mathrm{cl}} \Psi_{1}^{\mathrm{cl}}\right]-\frac{1}{2}\left[\Psi_{1}^{\mathrm{cl}} b_{0}^{+}\left(L_{0}^{+}\right)^{-1}(1-P)\left[\Psi_{1}^{\mathrm{cl}} \Psi_{1}^{\mathrm{cl}}\right]\right]\right) .
$$

A consistency condition for the existence of a solution to this equation is the vanishing of the inner product of both sides with $\langle\phi| c_{0}^{-}$for the three states $|\phi\rangle$ listed in (7.6). The inner product of the left hand side with all the states vanish since all of these states are BRST invariant. The inner product of the right hand side with the first two states in (7.6) vanish due to the vanishing of the correlation functions of odd number of $\partial Y$ 's and / or odd number of $\bar{\partial} Y$ 's. Since the last state in (7.6) is given by $\left|\Psi_{1}^{\mathrm{cl}}\right\rangle$, we need to check the vanishing of

$$
\begin{aligned}
A & \equiv\left\langle\Psi_{1}^{\mathrm{cl}}\right| c_{0}^{-} P\left(\left[\Psi_{1}^{\mathrm{cl}} \Psi_{1}^{\mathrm{cl}} \Psi_{1}^{\mathrm{cl}}\right]-3\left[\Psi_{1}^{\mathrm{cl}} b_{0}^{+}\left(L_{0}^{+}\right)^{-1}(1-P)\left[\Psi_{1}^{\mathrm{cl}} \Psi_{1}^{\mathrm{cl}}\right]\right]\right) \\
& =\left\{\Psi_{1}^{\mathrm{cl}} \Psi_{1}^{\mathrm{cl}} \Psi_{1}^{\mathrm{cl}} \Psi_{1}^{\mathrm{cl}}\right\}-3\left\{\Psi_{1}^{\mathrm{cl}} \Psi_{1}^{\mathrm{cl}} b_{0}^{+}\left(L_{0}^{+}\right)^{-1}(1-P)\left[\Psi_{1}^{\mathrm{cl}} \Psi_{1}^{\mathrm{cl}}\right]\right\} .
\end{aligned}
$$

Formally this can be regarded as the four point function of four $\Psi_{1}^{\mathrm{cl}}$ states, with the first term representing the contribution from the elementary vertex and the second term representing the contribution from the $\mathrm{s}, \mathrm{t}$ and $\mathrm{u}$ channel diagrams all of which are equal. However unlike the four point function which has divergences from collision of vertex operators, (7.11) is manifestly finite. To evaluate it we shall choose a local coordinate system of the type described in section 3 with large $\lambda$. We emphasize however that the choice of large $\lambda$ is a matter of convenience, but is in no way necessary.

Since the external momenta all vanish and are not generic, we can have ambiguities in applying the procedure of section 4, 5. Therefore we shall evaluate (7.11) directly. First we have

$$
\begin{aligned}
\left\{\Psi_{1}^{\mathrm{cl}} \Psi_{1}^{\mathrm{cl}} \Psi_{1}^{\mathrm{cl}} \Psi_{1}^{\mathrm{cl}}\right\} & =\frac{1}{2 \pi i} \int_{\mathcal{R}} d \sigma \wedge d \bar{\sigma}\left\langle\Psi_{1}^{\mathrm{cl}}(0) \Psi_{1}^{\mathrm{cl}}(1) \Psi_{1}^{\mathrm{cl}}(\infty) V(\sigma)\right\rangle \\
& =-\frac{1}{32 \pi i} \int_{\mathcal{R}} d \sigma \wedge d \bar{\sigma}\left[\sigma^{-2}+(\sigma-1)^{-2}+1\right]\left[\bar{\sigma}^{-2}+(\bar{\sigma}-1)^{-2}+1\right] \\
& =\frac{1}{32 \pi i} \int_{\mathcal{R}} d \sigma \wedge d \bar{\sigma}\left[\sigma^{-2}+(\sigma-1)^{-2}+1\right] \partial_{\bar{\sigma}}\left[\bar{\sigma}^{-1}+(\bar{\sigma}-1)^{-1}-\bar{\sigma}\right] \\
& =\frac{1}{32 \pi i} \int_{\partial \mathcal{R}_{s}+\partial \mathcal{R}_{u}+\partial \mathcal{R}_{t}} d \sigma\left[\sigma^{-2}+(\sigma-1)^{-2}+1\right]\left[\bar{\sigma}^{-1}+(\bar{\sigma}-1)^{-1}-\bar{\sigma}\right],
\end{aligned}
$$


where we have used $\partial \mathcal{R}=-\partial \mathcal{R}_{s}-\partial \mathcal{R}_{u}-\partial \mathcal{R}_{t}$. Since all the external states are identical, the contribution from $\partial \mathcal{R}_{u}, \partial \mathcal{R}_{s}$ and $\partial \mathcal{R}_{t}$ are identical. So we shall calculate the contribution from one of the boundaries $\partial \mathcal{R}_{u}$ around $\sigma=0$ and multiply the result by 3 . Now we see from (3.13) and (3.17) that in the large $\lambda$ limit, $\partial \mathcal{R}_{u}$ given by $|q|=1$ corresponds approximately to a circle around $\sigma=0$ of radius of order $\lambda^{-2}$. If we drop terms proportional to negative powers of $\lambda$, which will drop out when we take the $\lambda \rightarrow \infty$ limit, then the only contribution to (7.12) comes from the $\sigma^{-2} \bar{\sigma}^{-1}$ term in the integrand. This leads to

$$
\left\{\Psi_{1}^{\mathrm{cl}} \Psi_{1}^{\mathrm{cl}} \Psi_{1}^{\mathrm{cl}} \Psi_{1}^{\mathrm{cl}}\right\}=\frac{3}{32 \pi i} \int_{\partial \mathcal{R}_{u}} d \sigma \sigma^{-2} \bar{\sigma}^{-1} .
$$

Since the $\partial \mathcal{R}_{u}$ is not strictly a circle in the $\sigma$-plane, it will be more convenient to express the boundary term in the $q$ plane via the relation (3.17). This gives

$$
\left\{\Psi_{1}^{\mathrm{cl}} \Psi_{1}^{\mathrm{cl}} \Psi_{1}^{\mathrm{cl}} \Psi_{1}^{\mathrm{cl}}\right\}=-\frac{3}{32 \pi i} \int_{|\xi|=1 / \lambda^{2}} d \xi \frac{g^{\prime}(\xi)}{(1-g(\xi))^{2}} \frac{1}{1-\overline{g(\xi)}}
$$

where $\xi=q / \lambda^{2}$. Since the integral has to be evaluated at $|\xi|=1 / \lambda^{2}$, we can expand $g(\xi)$ in a power series in $\xi$ for large $\lambda$. Using (3.13) we have

$$
g(\xi)=1-\frac{\xi}{h_{3}^{\prime}(1)^{2}}+\mathcal{O}\left(\xi^{2}\right), \quad \frac{g^{\prime}(\xi)}{(1-g(\xi))^{2}}=\frac{\partial}{\partial \xi}\left(\frac{1}{1-g(\xi)}\right)=-\frac{h_{3}^{\prime}(1)^{2}}{\xi^{2}}+\mathcal{O}(1) .
$$

Substituting this into (7.14) we get

$$
\left\{\Psi_{1}^{\mathrm{cl}} \Psi_{1}^{\mathrm{cl}} \Psi_{1}^{\mathrm{cl}} \Psi_{1}^{\mathrm{cl}}\right\} \simeq \frac{3}{16} \lambda^{4}\left|h_{3}^{\prime}(1)\right|^{4},
$$

where $\simeq$ means that we have dropped terms involving inverse powers of $\lambda$.

The second term on the right hand side of (7.11) is given by

$$
\begin{aligned}
& -3\left\{\Psi_{1}^{\mathrm{cl}} \Psi_{1}^{\mathrm{cl}} b_{0}^{+}\left(L_{0}^{+}\right)^{-1}(1-P)\left[\Psi_{1}^{\mathrm{cl}} \Psi_{1}^{\mathrm{cl}}\right]\right\} \\
= & -3 \sum_{p, q}\left\{\Psi_{1}^{\mathrm{cl}} \Psi_{1}^{\mathrm{cl}} \xi_{p}\right\}\left\langle\xi_{p}^{\mathrm{c}}\left|b_{0}^{+}\left(L_{0}^{+}\right)^{-1}(1-P) \delta_{L_{0}^{-}} b_{0}^{-}\right| \xi_{q}^{c}\right\rangle\left\{\xi_{q} \Psi_{1}^{\mathrm{cl}} \Psi_{1}^{\mathrm{cl}}\right\},
\end{aligned}
$$

where $\left|\xi_{p}\right\rangle$ denote a complete basis of ghost number 2 states in $\mathcal{H}$ and $\left|\xi_{p}^{c}\right\rangle$ is a conjugate basis of ghost number 4 states in $c_{0}^{-} \mathcal{H}$ satisfying

$$
\left\langle\xi_{p}^{c} \mid \xi_{q}\right\rangle=\delta_{p q}=\left\langle\xi_{q} \mid \xi_{p}^{c}\right\rangle, \quad \sum_{p}\left|\xi_{p}\right\rangle\left\langle\xi_{p}^{c}\left|=\mathbf{1}, \quad \sum_{p}\right| \xi_{p}^{c}\right\rangle\left\langle\xi_{p}\right|=\mathbf{1}
$$

where it is understood that the $\mathbf{1}$ in the middle equation is the projection operator into ghost number 2 states in $\mathcal{H}$ and the $\mathbf{1}$ in the last equation is the projection operator into ghost number 4 states in $c_{0}^{-} \mathcal{H}$. In arriving at the right hand side of (7.17), we have replaced $\left[\Psi_{1}^{\mathrm{cl}} \Psi_{1}^{\mathrm{cl}}\right]$ by $b_{0}^{-} c_{0}^{-}\left[\Psi_{1}^{\mathrm{cl}} \Psi_{1}^{\mathrm{cl}}\right]$ using the fact that $\left[\Psi_{1}^{\mathrm{cl}} \Psi_{1}^{\mathrm{cl}}\right] \in \mathcal{H}$, and then inserted a sum over complete set of states between $b_{0}^{-}$and $c_{0}^{-}$. Now from (3.2) we see that if $\xi_{p}$ has $L_{0}^{+}$eigenvalue $\Delta_{p}$, then for dimension 0 primaries $A, B$, the $\left\{A B \xi_{p}\right\}$ carries a factor of $\left|d w_{3} / d z\right|_{z=z_{3}}^{-\Delta_{p}}=\left(\lambda\left|h_{3}^{\prime}(1)\right|\right)^{-\Delta_{p}}$. Since $\Psi_{1}^{\mathrm{cl}}$ is a dimension 0 primary, it follows that for large $\lambda$ we can restrict the sum over $p, q$ in (7.17) to states with $\Delta_{p} \leq 0$. Now the contributions 
from the $\Delta_{p}=0$ states are already removed by the projection operator $(1-P)$, therefore we have to focus on states with $\Delta_{p}<0$. The only such state is the ground state with $\Delta_{p}=-2$, giving

$$
\left|\xi_{p}\right\rangle=\left|\xi_{q}\right\rangle=c_{1} \bar{c}_{1}|0\rangle, \quad\left|\xi_{p}^{c}\right\rangle=\left|\xi_{q}^{c}\right\rangle=-c_{0} \bar{c}_{0} c_{1} \bar{c}_{1}|0\rangle
$$

Therefore we have

$$
\begin{aligned}
& \left\{\Psi_{1}^{\mathrm{cl}} \Psi_{1}^{\mathrm{cl}} \xi_{p}\right\}=-\frac{1}{4}\left(\lambda\left|h_{3}^{\prime}(1)\right|\right)^{2}, \quad\left\{\xi_{q} \Psi_{1}^{\mathrm{cl}} \Psi_{1}^{\mathrm{cl}}\right\}=\left\{\Psi_{1}^{\mathrm{cl}} \Psi_{1}^{\mathrm{cl}} \xi_{q}\right\}=-\frac{1}{4}\left(\lambda\left|h_{3}^{\prime}(1)\right|\right)^{2} \\
& \left\langle\xi_{p}^{c}\left|b_{0}^{+}\left(L_{0}^{+}\right)^{-1}(1-P) \delta_{L_{0}^{-}} b_{0}^{-}\right| \xi_{q}^{c}\right\rangle=1 .
\end{aligned}
$$

This gives

$$
-3\left\{\Psi_{1}^{\mathrm{cl}} \Psi_{1}^{\mathrm{cl}} b_{0}^{+}\left(L_{0}^{+}\right)^{-1}\left[\Psi_{1}^{\mathrm{cl}} \Psi_{1}^{\mathrm{cl}}\right]\right\} \simeq-\frac{3}{16}\left(\lambda\left|h_{3}^{\prime}(1)\right|\right)^{4} .
$$

(7.21) exactly cancels (7.16). Therefore (7.11) gives

$$
A=0 \text {. }
$$

This in turn shows that there is no obstruction to extending the solution to order $\mu^{3}$. This is equivalent to proving the vanishing of the $\beta$-function for the marginal deformation $V$ to order $\mu^{3}$ without having to introduce ultraviolet cut-off at any step.

Earlier explicit analysis of marginal deformations in closed string field theory [38] examined the solution to second order and therefore did not encounter dependence on the local coordinate $\lambda h_{3}(z)$ in the intermediate steps of the calculation. As stated below (7.11), and noted in [38], at order $\lambda^{3}$ the existence of the solution formally requires the vanishing of the four point function of marginal operators. However the corresponding integrand diverges when vertex operators collide and one has to regularize it. In contrast, string field theory gives finite result by treating $\left(L_{0}^{+}\right)^{-1}$ correctly.

We now turn to the computation of the spectrum of the deformed theory to order $\mu^{2}$. For this we need to study the fluctuation of the string field around the new background. Let us define:

$$
|\Phi\rangle=|\Psi\rangle-\left|\Psi^{\mathrm{cl}}\right\rangle
$$

Then the equation of motion (7.1) to linear order in $\Phi$ is given by:

$$
Q_{B}|\Phi\rangle+\sum_{N=1}^{\infty} \frac{1}{N !}\left[\left(\Psi^{\mathrm{cl}}\right)^{N} \Phi\right]=0 .
$$

Let us for definiteness consider the tachyon state carrying momentum $n / R$ along the compact direction and $k$ along the non-compact directions. Starting with the leading order solution to (7.24), we shall compute systematic corrections to the solution following the procedure described in [35]. We shall denote by $\left|\Phi_{\ell}\right\rangle$ the full solution to order $\mu^{\ell}$. To order $\mu^{0}$ the solution to (7.24) takes the form:

$$
\left|\Phi_{0}\right\rangle=\left|\phi_{0}\right\rangle, \quad \phi_{0}=c \bar{c} e^{i k_{(0)} \cdot X} e^{i n Y / R}, \quad k_{(0)}^{2}=-m^{2}-\frac{n^{2}}{R^{2}}=4-\frac{n^{2}}{R^{2}},
$$

where we are using the standard state operator correspondence: $\left|\phi_{0}\right\rangle=\phi_{0}(0)|0\rangle$. 
For subsequent analysis we need to introduce a projection operator $\mathcal{P}$ that projects to states which carry momentum $k=k_{(0)}+\mathcal{O}(\mu)$ along non-compact directions, $n / R$ along the compact direction and have $L_{0}^{+}=\mathcal{O}(\mu)$. It is easy to verify that in this case only vertex operators invariant under $\mathcal{P}$ are those of the form $c \bar{c} e^{i k . X} e^{i n Y / R}$. We normalize $\left|\Phi_{\ell}\right\rangle$ such that

$$
\left|\phi_{\ell}\right\rangle \equiv \mathcal{P}\left|\Phi_{\ell}\right\rangle=c_{1} \bar{c}_{1} e^{i k_{(\ell)} \cdot X}(0) e^{i n Y / R}(0)|0\rangle, \quad k_{(\ell)}=k_{(0)}+\mathcal{O}(\mu) .
$$

As discussed in [35], since $k_{(\ell)}$ is expected to be different from $k_{(0)}$ due to a change in the mass, it is not convenient to use the solution $\left|\Phi_{0}\right\rangle$ and correct it to obtain $\left|\Phi_{\ell}\right\rangle$. Instead we begin with $\left|\phi_{\ell}\right\rangle$ given in (7.26) as the seed solution and correct it to order $\mu^{\ell}$ to determine $\left|\Phi_{\ell}\right\rangle$ and $k_{(\ell)}$.

We begin with the ansatz for the solution $\left|\Phi_{1}\right\rangle$ :

$$
\left|\Phi_{1}\right\rangle=\left|\phi_{1}\right\rangle+\mathcal{O}(\mu), \quad \phi_{1}=c \bar{c} e^{i k_{(1)} \cdot X} e^{i n Y / R}, \quad k_{(1)}^{2}=k_{(0)}^{2}+a_{1} \mu,
$$

where the constant $a_{1}$ will be determined shortly. Taking (7.27) as the leading order solution, we can substitute this into the right hand side of (7.24) to get a solution to order $\mu$ :

$$
\left|\Phi_{1}\right\rangle=-\mu \frac{b_{0}^{+}}{L_{0}^{+}}(1-\mathcal{P})\left[\Psi_{1}^{\mathrm{cl}} \phi_{1}\right]+\left|\phi_{1}\right\rangle
$$

provided $\left|\phi_{1}\right\rangle$ satisfies:

$$
Q_{B}\left|\phi_{1}\right\rangle=-\mu \mathcal{P}\left[\Psi_{1}^{\mathrm{cl}} \phi_{1}\right]+\mathcal{O}\left(\mu^{2}\right)
$$

If we define

$$
\tilde{\phi}_{1}=c \bar{c} e^{-i k_{(1)} \cdot X} e^{-i n Y / R},
$$

satisfying the normalization

$$
\left\langle\tilde{\phi}_{1}\left|c_{0} \bar{c}_{0}\right| \phi_{1}\right\rangle=-1,
$$

and take the inner product of $\left\langle\tilde{\phi}_{1}\right| c_{0}^{-}$with (7.29), we get

$$
\left\langle\tilde{\phi}_{1}\left|c_{0}^{-} Q_{B}\right| \phi_{1}\right\rangle=-\mu\left\langle\tilde{\phi}_{1}\left|c_{0}^{-}\right|\left[\Psi_{1}^{\mathrm{cl}} \phi_{1}\right]\right\rangle=-\mu\left\{\tilde{\phi}_{1} \Psi_{1}^{\mathrm{cl}} \phi_{1}\right\}=-\mu\left\langle\tilde{\phi}_{1}|c \bar{c} \partial Y \bar{\partial} Y(1)| \phi_{1}\right\rangle+\mathcal{O}\left(\mu^{2}\right),
$$

where in the last step we have used the fact that $\Psi_{1}^{\mathrm{cl}}$ is a dimension 0 primary and $\phi_{1}$ and $\tilde{\phi}_{1}$ are primaries of dimension of order $\mu$. Only the $\left(c_{0} L_{0}+\bar{c}_{0} \bar{L}_{0}\right)$ term in $Q_{B}$ contributes to the left hand side. This gives

$$
-\frac{1}{4}\left(k_{(1)}^{2}-4+n^{2} R^{-2}\right)=-\frac{n^{2}}{4 R^{2}} \mu \quad \Rightarrow \quad k_{(1)}^{2}=4-n^{2} R^{-2}(1-\mu) .
$$

We now turn to order $\mu^{2}$ computation. We begin with the ansatz

$$
\left|\Phi_{2}\right\rangle=\left|\phi_{2}\right\rangle+\mathcal{O}(\mu)
$$

with $\left|\phi_{2}\right\rangle$ as defined in (7.26), and solve the equations of motion (7.24) iteratively to get successive order solutions:

$$
\left|\Phi_{2}\right\rangle=-\mu \frac{b_{0}^{+}}{L_{0}^{+}}(1-\mathcal{P})\left[\Psi_{1}^{\mathrm{cl}} \phi_{2}\right]+\left|\phi_{2}\right\rangle+\mathcal{O}\left(\mu^{2}\right),
$$


and

$$
\begin{aligned}
\left|\Phi_{2}\right\rangle= & -\frac{b_{0}^{+}}{L_{0}^{+}}(1-\mathcal{P})\left(\mu\left[\Psi_{1}^{\mathrm{cl}} \phi_{2}\right]-\mu^{2}\left[\Psi_{1}^{\mathrm{cl}} \frac{b_{0}^{+}}{L_{0}^{+}}(1-\mathcal{P})\left[\Psi_{1}^{\mathrm{cl}} \phi_{2}\right]\right]+\mu^{2}\left[\Psi_{2}^{\mathrm{cl}} \phi_{2}\right]+\frac{\mu^{2}}{2}\left[\Psi_{1}^{\mathrm{cl}} \Psi_{1}^{\mathrm{cl}} \phi_{2}\right]\right) \\
& +\left|\phi_{2}\right\rangle+\mathcal{O}\left(\mu^{3}\right),
\end{aligned}
$$

provided $\left|\phi_{2}\right\rangle$ satisfies, to order $\mu^{2}$,

$$
\begin{aligned}
Q_{B}\left|\phi_{2}\right\rangle= & -\mathcal{P}\left(\mu\left[\Psi_{1}^{\mathrm{cl}} \phi_{2}\right]-\mu^{2}\left[\Psi_{1}^{\mathrm{cl}} \frac{b_{0}^{+}}{L_{0}^{+}}(1-\mathcal{P})\left[\Psi_{1}^{\mathrm{cl}} \phi_{2}\right]\right]\right. \\
& \left.-\frac{\mu^{2}}{2}\left[\left(\frac{b_{0}^{+}}{L_{0}^{+}}(1-P)\left[\Psi_{1}^{\mathrm{cl}} \Psi_{1}^{\mathrm{cl}}\right]\right) \phi_{2}\right]+\frac{\mu^{2}}{2}\left[\Psi_{1}^{\mathrm{cl}} \Psi_{1}^{\mathrm{cl}} \phi_{2}\right]\right) .
\end{aligned}
$$

In the second line we have used the expression for $\left|\Psi_{2}^{\mathrm{cl}}\right\rangle$ given in (7.4), (7.7). Defining

$$
\tilde{\phi}_{2}=c \bar{c} e^{-i k_{(2)} \cdot X} e^{-i n Y / R},
$$

and taking the inner product of $\left\langle\tilde{\phi}_{2}\right| c_{0}^{-}$with (7.37), using the analog of (7.31) with $\phi_{1}, \tilde{\phi}_{1}$ replaced by $\phi_{2}, \tilde{\phi}_{2}$, we get

$$
\begin{aligned}
-\frac{1}{4}\left(k_{(2)}^{2}-4+n^{2} R^{-2}\right)= & -\mu\left\{\tilde{\phi}_{2} \Psi_{1}^{\mathrm{cl}} \phi_{2}\right\}+\mu^{2}\left\{\tilde{\phi}_{2} \Psi_{1}^{\mathrm{cl}} \frac{b_{0}^{+}}{L_{0}^{+}}(1-\mathcal{P})\left[\Psi_{1}^{\mathrm{cl}} \phi_{2}\right]\right\} \\
& +\frac{\mu^{2}}{2}\left\{\tilde{\phi}_{2} \phi_{2}\left(\frac{b_{0}^{+}}{L_{0}^{+}}(1-P)\left[\Psi_{1}^{\mathrm{cl}} \Psi_{1}^{\mathrm{cl}}\right]\right)\right\}-\frac{\mu^{2}}{2}\left\{\tilde{\phi}_{2} \phi_{2} \Psi_{1}^{\mathrm{cl}} \Psi_{1}^{\mathrm{cl}}\right\} .
\end{aligned}
$$

We shall now evaluate the different terms appearing on the right hand side of (7.39). First of all we have:

$$
\begin{aligned}
\mu\left\{\tilde{\phi}_{2} \Psi_{1}^{\mathrm{cl}} \phi_{2}\right\} & =\frac{\mu}{4} n^{2} R^{-2}\left(\lambda\left|h_{3}^{\prime}(1)\right|\right)^{-k_{(2)}^{2}+4-n^{2} R^{-2}} \\
& =\frac{\mu}{4} n^{2} R^{-2}\left\{1-\left(k_{(2)}^{2}-4+n^{2} R^{-2}\right) \ln \left(\lambda\left|h_{3}^{\prime}(1)\right|\right)\right\}+\mathcal{O}\left(\mu^{3}\right),
\end{aligned}
$$

where the $\left(\lambda\left|h_{3}^{\prime}(1)\right|\right)^{-k_{(2)}^{2}+4-n^{2} R^{-2}}$ factor arises from the fact that the states $\phi_{2}, \tilde{\phi}_{2}$ have $L_{0}^{+}$eigenvalues $\Delta=\left(k_{(2)}^{2}-4+n^{2} R^{-2}\right) / 2$ and therefore $\left\{\tilde{\phi}_{2} \Psi_{1}^{\text {cl }} \phi_{2}\right\}$ will carry factors of $\left|d w_{3} / d z\right|_{z=z_{i}}^{-\Delta}$ for $i=1,3$. Using (3.1), (3.2) and (3.4) we get $\left(d w_{1} / d z\right)_{z=z_{1}}=$ $\left.\left(d w_{3} / d z\right)\right|_{z=z_{3}}=\lambda h_{3}^{\prime}(1)$. To evaluate the right hand side of $(7.40)$ to order $\mu^{2}$, we can replace $k_{(2)}$ by $k_{(1)}$ at the cost of making an error of order $\mu^{2}$. Using (7.33) we get

$$
-\mu\left\{\tilde{\phi}_{2} \Psi_{1}^{\mathrm{cl}} \phi_{2}\right\}=-\frac{\mu}{4} n^{2} R^{-2}+\frac{\mu^{2}}{4} n^{4} R^{-4} \ln \left(\lambda\left|h_{3}^{\prime}(1)\right|\right)+\mathcal{O}\left(\mu^{3}\right) .
$$

The evaluation of the last three terms on the right hand side of (7.39) can be simplified by noting that since all these terms already have explicit factors of $\mu^{2}$, we can replace the momenta $k_{(2)}$ by $k_{(0)}$ given in (7.25). This makes all the external states on-shell.

Let us begin with the evaluation of the last term in (7.39). This has the same expression as the on-shell four point function of the states $\phi, \tilde{\phi}, \Psi_{1}^{\mathrm{cl}}$ and $\Psi_{1}^{\mathrm{cl}}$, except that the integration 
over the moduli runs over the region $\mathcal{R}$ introduced in section 3.1 instead of the whole complex plane. The integrand for the on-shell amplitude is given by

$$
\begin{aligned}
\mathcal{I} & \equiv \frac{1}{2 \pi i}\left\langle c \bar{c} \partial Y \bar{\partial} Y(0) c \bar{c} e^{i k_{(0)} \cdot X} e^{i n Y / R}(\infty) c \bar{c} e^{-i k_{(0)} \cdot X} e^{-i n Y / R}(1) \partial Y \bar{\partial} Y(\sigma)\right\rangle \\
& =-\frac{1}{2 \pi i}\left\{\frac{1}{2 \sigma^{2}}-\frac{n^{2}}{4 R^{2}} \frac{1}{\sigma-1}\right\}\left\{\frac{1}{2 \bar{\sigma}^{2}}-\frac{n^{2}}{4 R^{2}} \frac{1}{\bar{\sigma}-1}\right\} .
\end{aligned}
$$

Therefore we have

$$
-\frac{\mu^{2}}{2}\left\{\tilde{\phi}_{2} \phi_{2} \Psi_{1}^{\mathrm{cl}} \Psi_{1}^{\mathrm{cl}}\right\}=-\frac{\mu^{2}}{2} \int_{\mathcal{R}} d^{2} \sigma \mathcal{I} \equiv I_{1}+I_{2}+I_{3}
$$

where

$$
\begin{gathered}
I_{1}=\frac{\mu^{2}}{16 \pi i} \int_{\mathcal{R}} d^{2} \sigma \sigma^{-2} \bar{\sigma}^{-2}, \\
I_{2}=-\frac{\mu^{2}}{32 \pi i} n^{2} R^{-2} \int_{\mathcal{R}} d^{2} \sigma\left\{\sigma^{-2}(\bar{\sigma}-1)^{-1}+\bar{\sigma}^{-2}(\sigma-1)^{-1}\right\},
\end{gathered}
$$

and

$$
I_{3}=\frac{\mu^{2}}{64 \pi i} n^{4} R^{-4} \int_{\mathcal{R}} d^{2} \sigma(\sigma-1)^{-1}(\bar{\sigma}-1)^{-1}
$$

While the analysis can be carried out for any choice of local coordinate system encoded in the functions $\lambda h_{i}(z)$ introduced in section 3.1, we shall simplify our analysis by taking $\lambda$ to be large. In this case it follows from (3.10), (3.17) and (3.20) that the excluded regions $|q| \leq 1$, denoted by $\mathcal{R}_{s}, \mathcal{R}_{u}$ and $\mathcal{R}_{t}$ in section 3.1 , correspond to small regions around 1,0 and a region outside a large radius respectively. Therefore for large $\lambda$ we can ignore the excluded regions $\mathcal{R}_{s}, \mathcal{R}_{t}$ and/or $\mathcal{R}_{u}$ as long as the integrands do not encounter any divergence. ${ }^{11}$ For example in $I_{1}$ we can ignore the excluded regions $\mathcal{R}_{s}$ and $\mathcal{R}_{t}$ since the integrand does not have any divergence from 1 and $\infty$, but cannot ignore the excluded region $\mathcal{R}_{u}$ around the origin. On the other hand in $I_{3}$ we can ignore the excluded region $\mathcal{R}_{u}$ around the origin, but cannot ignore $\mathcal{R}_{s}$ and $\mathcal{R}_{t}$.

Let us begin with the evaluation of $I_{1}$. Since the only excluded region in this expression is a small region $\mathcal{R}_{u}$ around 0 , we can express this as

$$
I_{1}=-\frac{\mu^{2}}{16 \pi i} \int_{\mathcal{R}} d \sigma \wedge d \bar{\sigma} \frac{\partial}{\partial \bar{\sigma}}\left(\sigma^{-2} \bar{\sigma}^{-1}\right)=-\frac{\mu^{2}}{16 \pi i} \int_{\partial \mathcal{R}_{u}} d \sigma \sigma^{-2} \bar{\sigma}^{-1}
$$

where we have used $\partial \mathcal{R}=-\partial \mathcal{R}_{u}$. This has the same structure as (7.13) and can be evaluated using identical procedure, leading to the analog of (7.16):

$$
I_{1} \simeq-\frac{\mu^{2}}{8} \lambda^{4}\left|h_{3}^{\prime}(1)\right|^{4}
$$

where $\simeq$ denotes equality up to terms containing inverse powers of $\lambda$.

\footnotetext{
${ }^{11}$ Note that we are not taking the large $\lambda$ limit of individual terms, but dropping terms with inverse powers of $\lambda$ in anticipation of the fact that eventually we shall take the $\lambda \rightarrow \infty$ limit after adding all the terms. In this limit the terms with inverse powers of $\lambda$ will drop out. We are however perfectly entitled to keep $\lambda$ finite and add up the contribution from all the terms.
} 
$I_{2}$ can be evaluated by expressing this as

$$
I_{2}=\frac{\mu^{2}}{32 \pi i} n^{2} R^{-2} \int_{\mathcal{R}} d^{2} \sigma\left\{\frac{\partial}{\partial \sigma}\left(\sigma^{-1}(\bar{\sigma}-1)^{-1}\right)+\frac{\partial}{\partial \bar{\sigma}}\left(\bar{\sigma}^{-1}(\sigma-1)^{-1}\right)\right\} .
$$

We can evaluate this using integration by parts, picking up boundary contributions from $\partial \mathcal{R}$. The only boundary that contributes is the boundary $-\partial \mathcal{R}_{s}$ around $\sigma=1$. This gives

$$
I_{2}=\frac{\mu^{2}}{8} n^{2} R^{-2}
$$

$I_{3}$ can be evaluated by noting that the integral has logarithmic divergence both near 1 and $\infty$, and therefore we need to use eqs. (3.10) and (3.20) to determine the cut-off on $\sigma$ integral by identifying the $|q|=1$ curves. Using (3.13) we see that in the $\sigma=y_{4}$ plane these curves are at

$$
|\sigma-1| \simeq\left|h_{3}^{\prime}(1)\right|^{-2} \lambda^{-2}
$$

and

$$
|\sigma| \simeq \lambda^{2}\left|h_{3}^{\prime}(1)\right|^{2}
$$

respectively. Since the integrals are at most logarithmically divergent we do not need to know the corrections to these curves. This gives

$$
I_{3} \simeq \frac{\mu^{2}}{64 \pi i} n^{4} R^{-4} \int_{\left|h_{3}^{\prime}(1)\right|^{-2} \lambda^{-2} \leq|\sigma-1| \leq \lambda^{2}\left|h_{3}^{\prime}(1)\right|^{2}} d^{2} \sigma|\sigma-1|^{-2}=-\frac{\mu^{2}}{4} n^{4} R^{-4} \ln \left(\lambda\left|h_{3}^{\prime}(1)\right|\right) .
$$

Using the values of $I_{1}, I_{2}, I_{3}$ determined above, we finally get from (7.43)

$$
-\frac{\mu^{2}}{2}\left\{\tilde{\phi}_{2} \phi_{2} \Psi_{1}^{\mathrm{cl}} \Psi_{1}^{\mathrm{cl}}\right\}=I_{1}+I_{2}+I_{3} \simeq-\frac{\mu^{2}}{8} \lambda^{4}\left|h_{3}^{\prime}(1)\right|^{4}+\frac{\mu^{2}}{8} \frac{n^{2}}{R^{2}}-\frac{\mu^{2}}{4} n^{4} R^{-4} \ln \left(\lambda\left|h_{3}^{\prime}(1)\right|\right) .
$$

Let us now turn to the contribution from the third term on the right hand side of (7.39). Evaluation of this proceeds exactly as that of (7.17) with the only difference that the first two factors of $\Psi_{1}^{\mathrm{cl}}$ are replaced by $\tilde{\phi}_{2} \phi_{2}$. The first equation in (7.20) is replaced by

$$
\left\{\tilde{\phi}_{2} \phi_{2} \xi_{p}\right\}=-\left(\lambda\left|h_{3}^{\prime}(1)\right|\right)^{2}
$$

This gives the analog of (7.21):

$$
I_{4} \equiv \frac{\mu^{2}}{2}\left\{\tilde{\phi}_{2} \phi_{2}\left(\frac{b_{0}^{+}}{L_{0}^{+}}(1-P) \delta_{L_{0}^{-}} b_{0}^{-} c_{0}^{-}\left[\Psi_{1}^{\mathrm{cl}} \Psi_{1}^{\mathrm{cl}}\right]\right)\right\} \simeq \frac{\mu^{2}}{8}\left(\lambda\left|h_{3}^{\prime}(1)\right|\right)^{4} .
$$

The contribution from the second term on the right hand side of (7.39) vanishes in the large $\lambda$ limit due to the following reason. As already argued before, for evaluation of this term we can set $k_{(2)}=k_{(0)}$. In this case momentum conservation forces the intermediate state to carry a factor of $e^{i k_{(0)} \cdot X+i n Y / R}$ which has $L_{0}^{+}=2$. Therefore the lowest $L_{0}^{+}$eigenvalue state propagating in this channel is $c \bar{c} e^{i k_{(0)} \cdot X+i n Y / R}$ with $L_{0}^{+}=0$. The contribution 
from this state is projected out by the $(1-\mathcal{P})$ operator insertion. Therefore the contribution comes only from states with positive $L_{0}^{+}$eigenvalue, leading to terms with negative powers of $\lambda$. Such terms will vanish in the large $\lambda$ limit. Therefore

$$
\left\{\tilde{\phi}_{2} \Psi_{1}^{\mathrm{cl}} \frac{b_{0}^{+}}{L_{0}^{+}}(1-\mathcal{P})\left[\Psi_{1}^{\mathrm{cl}} \phi_{2}\right]\right\} \simeq 0
$$

We now have from (7.39), (7.41), (7.54), (7.56) and (7.57),

$$
\begin{aligned}
-\frac{1}{4}\left(k_{(2)}^{2}-4+n^{2} R^{-2}\right) & \simeq-\mu\left\{\tilde{\phi}_{2} \Psi_{1}^{\mathrm{cl}} \phi_{2}\right\}+I_{1}+I_{2}+I_{3}+I_{4} \\
& =-\left(\frac{\mu}{4}-\frac{\mu^{2}}{8}\right) n^{2} R^{-2} .
\end{aligned}
$$

This gives

$$
k_{(2)}^{2}=4-n^{2} R^{-2}\left(1-\mu+\frac{\mu^{2}}{2}\right) .
$$

Therefore the mass ${ }^{2}$ of the state to order $\mu^{2}$ is given by

$$
m^{2}=-k_{(2)}^{2}=-4+n^{2} R^{-2}\left(1-\mu+\frac{\mu^{2}}{2}\right) .
$$

This is consistent with the expectation that the marginal deformation induces a deformation of the radius $R$ of the compact direction. Note that we have arrived at this result without encountering any divergence from collision of the pair of marginal operators or of the marginal operator with the vertex operator of the tachyon. The $\mu$ dependent terms can be regarded as an expansion of $e^{-\mu}$ but we do not have a compelling reason to believe that this pattern will continue to hold at higher order. In particular different versions of string field theory are expected to give different results related by a redefinition of $\mu$.

\subsection{Heterotic string theory}

We shall now repeat the analysis of the previous section for heterotic string theory. The marginal deformation corresponds to switching on NS sector string field. For definiteness we shall take the states, whose mass shift we compute, also to be in the NS sector, but the generalization to Ramond sector states is straightforward. Since in the NS sector the structure of heterotic string field theory is identical to that of bosonic string field theory, most of the analysis takes identical form. For this reason, we shall mention only the differences.

The first difference is in the form of the leading order classical solution describing the shifted vacuum. We take this to be of the form:

$$
\mu\left|\Psi_{1}^{\mathrm{cl}}\right\rangle=\mu c_{1} \bar{c}_{1} e^{-\phi}(0) V(0)|0\rangle, \quad V(z, \bar{z})=-2 \chi(z) \bar{\partial} Y(\bar{z})
$$

where we have used the notation of section 2 for various fields. The form of (7.4) and (7.5) remain unchanged. The possible list of $L_{0}^{+}=0$ states in $\mathcal{H}$ which have the correct total 
ghost and picture numbers and $(Y \rightarrow-Y, \chi \rightarrow-\chi)$ symmetry for having non-zero inner product with $c_{0}^{-}\left[\Psi_{1}^{\mathrm{cl}} \Psi_{1}^{\mathrm{cl}}\right]$ is

$$
c_{1} \eta_{-1}|0\rangle, \quad \bar{c}_{1} \bar{c}_{-1} c_{1} \xi_{-1} e^{-2 \phi}(0)|0\rangle, \quad c_{1} \bar{c}_{1} e^{-\phi}(0) V(0)|0\rangle .
$$

However all of these actually have vanishing inner product with $c_{0}^{-}\left[\Psi_{1}^{\mathrm{cl}} \Psi_{1}^{\mathrm{cl}}\right]$ due to separate ghost charge conservation in holomorphic and anti-holomorphic sectors and / or the $\chi \rightarrow$ $-\chi$ symmetry. Therefore we can take $\left|\psi_{2}^{\mathrm{cl}}\right\rangle=0$ as in bosonic string theory. Showing that the solution extends to order $\mu^{3}$ is also straightforward, but we shall not describe it here since we only use the solution to order $\mu^{2}$.

Analysis of the fluctuations around the deformed vacuum also proceeds as in the case of bosonic string theory. We replace the tachyon carrying spatial momentum $k$ and internal momentum $n / R$ by a massless field with the same momenta. The ansatz for $\phi_{\ell}$ and $\tilde{\phi}_{\ell}$, replacing (7.26), (7.30) and (7.38) takes the form:

$$
\begin{gathered}
\phi_{\ell}=-2 c \bar{c} e^{-\phi} \psi^{1} \bar{\partial} X^{2} e^{i k_{(\ell)} \cdot X} e^{i n Y / R}, \quad \tilde{\phi}_{\ell}=-2 c \bar{c} e^{-\phi} \psi^{1} \bar{\partial} X^{2} e^{-i k_{(\ell)} \cdot X} e^{-i n Y / R}, \\
k_{(\ell)}^{2}=-n^{2} R^{-2}+\mathcal{O}(\mu), \quad \ell=0,1,2,
\end{gathered}
$$

satisfying the normalization condition:

$$
\left\langle\tilde{\phi}_{\ell}\left|c_{0} \bar{c}_{0}\right| \phi_{\ell}\right\rangle=-1
$$

$k_{(\ell)}$ 's have vanishing spatial components along 1 and 2 directions. The rest of the analysis proceeds as in bosonic string theory. To first order in $\mu$ we get the analog of (7.32):

$$
\left\langle\tilde{\phi}_{1}\left|c_{0}^{-} Q_{B}\right| \phi_{1}\right\rangle=-\mu\left\{\tilde{\phi}_{1} \Psi_{1}^{\mathrm{cl}} \phi_{1}\right\}=2 \mu\left\langle\tilde{\phi}_{1}\left|\mathcal{X}(p) c \bar{c} e^{-\phi} \chi \bar{\partial} Y(1)\right| \phi_{1}\right\rangle+\mathcal{O}\left(\mu^{2}\right),
$$

where $p$ is the PCO location on the NS-NS-NS interaction vertex chosen according to the rules discussed in section 3.2. Explicit evaluation gives a result independent of $p$ :

$$
-\frac{1}{4}\left(k_{(1)}^{2}+n^{2} R^{-2}\right)=-\mu\left\{\tilde{\phi}_{1} \Psi_{1}^{\mathrm{cl}} \phi_{1}\right\}=-\frac{n^{2}}{4 R^{2}} \mu \quad \Rightarrow \quad k_{(1)}^{2}=-n^{2} R^{-2}(1-\mu) .
$$

At order $\mu^{2}$ we have the analog of (7.39):

$$
\begin{aligned}
-\frac{1}{4}\left(k_{(2)}^{2}+n^{2} R^{-2}\right)= & -\mu\left\{\tilde{\phi}_{2} \Psi_{1}^{\mathrm{cl}} \phi_{2}\right\}+\mu^{2}\left\{\tilde{\phi}_{2} \Psi_{1}^{\mathrm{cl}} \frac{b_{0}^{+}}{L_{0}^{+}}(1-\mathcal{P})\left[\Psi_{1}^{\mathrm{cl}} \phi_{2}\right]\right] \\
& +\frac{\mu^{2}}{2}\left\{\tilde{\phi}_{2} \phi_{2}\left(\frac{b_{0}^{+}}{L_{0}^{+}}(1-P)\left[\Psi_{1}^{\mathrm{cl}} \Psi_{1}^{\mathrm{cl}}\right]\right)\right\}-\frac{\mu^{2}}{2}\left\{\tilde{\phi}_{2} \phi_{2} \Psi_{1}^{\mathrm{cl}} \Psi_{1}^{\mathrm{cl}}\right\} .
\end{aligned}
$$

The first term on the right hand side can be evaluated exactly as in bosonic string theory, leading to the analog of (7.41):

$$
-\mu\left\{\tilde{\phi}_{2} \Psi_{1}^{\mathrm{cl}} \phi_{2}\right\}=-\frac{\mu}{4} n^{2} R^{-2}+\frac{\mu^{2}}{4} n^{4} R^{-4} \ln \left(\lambda\left|h_{3}^{\prime}(1)\right|\right)+\mathcal{O}\left(\mu^{3}\right) .
$$

In this case the contribution from the second and the third terms on the right hand side of (7.67) carry only negative powers of $\lambda$ since there are no states with $L_{0}^{+}<0$ and the 
contributions from the $L_{0}^{+}=0$ states are removed by the projection operators $(1-P)$ and $(1-\mathcal{P})$. Therefore we are left to evaluate the last term.

As in the bosonic string theory, while evaluating $\left\{\tilde{\phi}_{2} \phi_{2} \Psi_{1}^{\mathrm{cl}} \Psi_{1}^{\mathrm{cl}}\right\}$ we can take the external states to be on-shell. The integrand depends on the choice of PCO locations but under a change in PCO locations the integrand changes by a total derivative. Therefore we can adjust them at will in the interior of $\mathcal{R}$, as long as on $\partial \mathcal{R}$ they coincide with the PCO locations in $\partial \mathcal{R}_{s}, \partial \mathcal{R}_{t}$ and $\partial \mathcal{R}_{u}$, fixed by the arrangements described in section 3.2. Our strategy will be to take the PCO locations to coincide with the locations of the vertex operators $\Psi_{1}^{\mathrm{cl}}$ in the interior of $\mathcal{R}$, and at the boundary $\partial \mathcal{R}$ make them jump to the values they take inside $\mathcal{R}_{s}, \mathcal{R}_{u}$ and $\mathcal{R}_{t}$. The effect of this jump can be computed via vertical integration $[45,46]$. If we choose a different PCO assignment inside $\mathcal{R}$, the bulk integrand will change by a total derivative. However the result of vertical integration will also change, precisely cancelling this effect.

The effect of taking the PCO locations to the locations of $\Psi_{1}^{\mathrm{cl}}$ is to convert the $\Psi_{1}^{\mathrm{cl}}$ inserted at 0 to an unintegrated 0 picture vertex operator given in (2.11)

$$
\lim _{z \rightarrow 0} \mathcal{X}(z) \Psi_{1}^{\mathrm{cl}}(0)=c_{1} \bar{c}_{1} \partial Y \bar{\partial} Y(0)|0\rangle+\frac{1}{2} \eta \bar{c} e^{\phi} \chi \bar{\partial} Y(0)|0\rangle,
$$

and the $\Psi_{1}^{\mathrm{cl}}$ inserted at $\sigma$ to an integrated zero picture vertex operator

$$
-\partial Y \bar{\partial} Y(\sigma) \text {. }
$$

Using these we get

$$
-\frac{\mu^{2}}{2}\left\{\tilde{\phi}_{2} \phi_{2} \Psi_{1}^{\mathrm{cl}} \Psi_{1}^{\mathrm{cl}}\right\}=-\frac{\mu^{2}}{2} \int_{\mathcal{R}} \mathcal{I}+\mathbf{B}_{v}
$$

where

$$
\begin{aligned}
\mathcal{I}= & 4 \frac{1}{2 \pi i} d^{2} \sigma\left\langle c \bar{c} e^{-\phi} \psi^{1} \bar{\partial} X^{2} e^{-i k_{(0)} \cdot X} e^{-i n Y / R}(1) \partial Y \bar{\partial} Y(\sigma)\right. \\
& \left.\left(c \bar{c} \partial Y \bar{\partial} Y(0)+\frac{1}{2} \eta \bar{c} e^{\phi} \chi \bar{\partial} Y(0)\right) c \bar{c} e^{-\phi} \psi^{1} \bar{\partial} X^{2} e^{i k_{(0)} \cdot X} e^{i n Y / R}(\infty)\right\rangle,
\end{aligned}
$$

and $\mathbf{B}_{v}$ is the result of vertical integration that moves the PCOs from their locations at 0 and $\sigma$ to the required values on $\partial \mathcal{R}$ so that they coincide with the positions of the PCOs on $\partial \mathcal{R}_{s}, \partial \mathcal{R}_{t}$ and $\partial \mathcal{R}_{u}$ given in section 3.2. If we denote by $W_{1}$ and $W_{2}$ the final locations of the PCOs, and follow the convention that we first move the PCO at 0 to $W_{1}$ and then move the PCO at $\sigma$ to $W_{2}$, then this has the effect of replacing inside the correlation function $[45,46]$ the factor

$$
\mathcal{X}(0) \mathcal{X}(\sigma) d \sigma \wedge d \bar{\sigma}\left(-\oint_{\sigma} b(w) d w\right)\left(-\oint_{\sigma} \bar{b}(\bar{w}) d \bar{w}\right),
$$

by

$$
\begin{aligned}
& \left(d \sigma \oint_{\sigma} b(w) d w+d \bar{\sigma} \oint_{\sigma} \bar{b}(\bar{w}) d \bar{w}\right)\left\{\left(\xi(0)-\xi\left(W_{1}\right)\right) \mathcal{X}(\sigma)+\mathcal{X}\left(W_{1}\right)\left(\xi(\sigma)-\xi\left(W_{2}\right)\right)\right\} \\
& -\left\{\xi(\sigma)-\xi\left(W_{2}\right)\right\} d \sigma \frac{\partial W_{1}}{\partial \sigma} \partial \xi\left(W_{1}\right) .
\end{aligned}
$$


Only the terms involving $\oint_{\sigma} \bar{b}(\bar{w}) d \bar{w}$ survive after imposing ghost charge conservation in the anti-holomorphic sector. This gives

$$
\begin{aligned}
\mathbf{B}_{v}= & 2 \frac{\mu^{2}}{2 \pi i} \int_{\partial \mathcal{R}} d \bar{\sigma}\left\langle c \bar{c} e^{-\phi} \psi^{1} \bar{\partial} X^{2} e^{-i k_{(0)} \cdot X} e^{-i n Y / R}(1) c \bar{c} e^{-\phi} \psi^{1} \bar{\partial} X^{2} e^{i k_{(0)} \cdot X} e^{i n Y / R}(\infty)\right. \\
& {\left[-2\left\{\xi(0)-\xi\left(W_{1}\right)\right\}\left\{c \partial Y+\frac{1}{2} \eta e^{\phi} \chi\right\} \bar{\partial} Y(\sigma) c \bar{c} e^{-\phi} \chi \bar{\partial} Y(0)\right.} \\
& \left.\left.+4\left\{\xi(\sigma)-\xi\left(W_{2}\right)\right\} \mathcal{X}\left(W_{1}\right) c e^{-\phi} \chi \bar{\partial} Y(\sigma) c \bar{c} e^{-\phi} \chi \bar{\partial} Y(0)\right]\right\rangle
\end{aligned}
$$

First let us compute the contribution from the first term on the right hand side of (7.71). The $\phi$-charge conservation (or equivalently $\xi-\eta$ charge conservation) tells us that the term proportional to $\eta$ in the second line of (7.72) does not contribute. Evaluation of the rest of the correlator gives

$$
\mathcal{I}=-\frac{1}{2 \pi i}\left\{\frac{1}{2 \sigma^{2}}-\frac{n^{2}}{4 R^{2}} \frac{1}{\sigma-1}\right\}\left\{\frac{1}{2 \bar{\sigma}^{2}}-\frac{n^{2}}{4 R^{2}} \frac{1}{\bar{\sigma}-1}\right\} .
$$

This is identical to the integrand in (7.42). Therefore we get, using (7.54)

$$
-\frac{\mu^{2}}{2} \int_{\mathcal{R}} \mathcal{I} \simeq-\frac{\mu^{2}}{8} \lambda^{4}\left|h_{3}^{\prime}(1)\right|^{4}+\frac{\mu^{2}}{8} \frac{n^{2}}{R^{2}}-\frac{\mu^{2}}{4} n^{4} R^{-4} \ln \left(\lambda\left|h_{3}^{\prime}(1)\right|\right) .
$$

Next we turn to the analysis of $\mathbf{B}_{v}$ given in (7.75). Using the relation

$$
\partial \mathcal{R}=-\partial \mathcal{R}_{s}-\partial \mathcal{R}_{t}-\partial \mathcal{R}_{u}
$$

we can express $\mathbf{B}_{v}$ as

$$
\mathbf{B}_{v}=\mathbf{B}_{s}+\mathbf{B}_{t}+\mathbf{B}_{u}
$$

where

$$
\begin{aligned}
\mathbf{B}_{s, t, u}= & -2 \frac{\mu^{2}}{2 \pi i} \int_{\partial \mathcal{R}_{s, t, u}} d \bar{\sigma}\left\langle c \bar{c} e^{-\phi} \psi^{1} \bar{\partial} X^{2} e^{-i k_{(0)} \cdot X} e^{-i n Y / R}(1) c \bar{c} e^{-\phi} \psi^{1} \bar{\partial} X^{2} e^{i k_{(0)} \cdot X} e^{i n Y / R}(\infty)\right. \\
& {\left[-2\left\{\xi(0)-\xi\left(W_{1}\right)\right\}\left\{c \partial Y(\sigma)+\frac{1}{2} \eta e^{\phi} \chi(\sigma)\right\} \bar{\partial} Y(\sigma) c \bar{c} e^{-\phi} \chi \bar{\partial} Y(0)\right.} \\
& \left.\left.+4\left\{\xi(\sigma)-\xi\left(W_{2}\right)\right\} \mathcal{X}\left(W_{1}\right) c e^{-\phi} \chi \bar{\partial} Y(\sigma) c \bar{c} e^{-\phi} \chi \bar{\partial} Y(0)\right]\right\rangle .
\end{aligned}
$$

We shall furthermore express $\mathbf{B}_{u}$ as

$$
\mathbf{B}_{u}=\mathbf{B}_{u}^{\prime}+\mathbf{B}_{u}^{\prime \prime},
$$

where

$$
\begin{aligned}
\mathbf{B}_{u}^{\prime}= & -2 \frac{\mu^{2}}{2 \pi i} \int_{\partial \mathcal{R}_{u}} d \bar{\sigma}\left\langle c \bar{c} e^{-\phi} \psi^{1} \bar{\partial} X^{2} e^{-i k_{(0)} \cdot X} e^{-i n Y / R}(1) c \bar{c} e^{-\phi} \psi^{1} \bar{\partial} X^{2} e^{i k_{(0)} \cdot X} e^{i n Y / R}(\infty)\right. \\
& {\left.\left[-2\{\xi(0)-\xi(1)\}\left\{c \partial Y+\frac{1}{2} \eta e^{\phi} \chi\right\} \bar{\partial} Y(\sigma) c \bar{c} e^{-\phi} \chi \bar{\partial} Y(0)\right]\right\rangle }
\end{aligned}
$$


and

$$
\begin{aligned}
\mathbf{B}_{u}^{\prime \prime}= & -2 \frac{\mu^{2}}{2 \pi i} \int_{\partial \mathcal{R}_{u}} d \bar{\sigma}\left\langle c \bar{c} e^{-\phi} \psi^{1} \bar{\partial} X^{2} e^{-i k_{(0)} \cdot X} e^{-i n Y / R}(1) c \bar{c} e^{-\phi} \psi^{1} \bar{\partial} X^{2} e^{i k_{(0)} \cdot X} e^{i n Y / R}(\infty)\right. \\
& {\left[-2\left\{\xi(1)-\xi\left(W_{1}\right)\right\}\left\{c \partial Y+\frac{1}{2} \eta e^{\phi} \chi\right\} \bar{\partial} Y(\sigma) c \bar{c} e^{-\phi} \chi \bar{\partial} Y(0)\right.} \\
& \left.\left.+4\left\{\xi(\sigma)-\xi\left(W_{2}\right)\right\} \mathcal{X}\left(W_{1}\right) c e^{-\phi} \chi \bar{\partial} Y(\sigma) c \bar{c} e^{-\phi} \chi \bar{\partial} Y(0)\right]\right\rangle
\end{aligned}
$$

We have shown in appendix B that in the large $\lambda$ limit, $\mathbf{B}_{s}, \mathbf{B}_{t}$ and $\mathbf{B}_{u}^{\prime \prime}$ vanish so that

$$
\mathbf{B}_{v}=\mathbf{B}_{u}^{\prime} .
$$

Intuitively the vanishing of $\mathbf{B}_{s}, \mathbf{B}_{t}$ and $\mathbf{B}_{u}^{\prime \prime}$ may be understood as follows. Let us start with $\mathbf{B}_{s}$. For large $\lambda$ the integration region is near a degeneration where the points 0 and $\infty$ are on one sphere and the points $\sigma$ and 1 are on another sphere, with the two spheres connected by a narrow neck. In this case the dominant contribution comes from nearly on-shell states propagating along the neck. In the initial configuration the PCO at 0 lies on the first sphere and the PCO at $\sigma$ lies on the second sphere. In the final configuration one of the PCOs $\left(W_{1}\right)$ lies on the first sphere and the other PCO $\left(W_{2}\right)$ lies on the second sphere. $\mathbf{B}_{s}$ describes the effect of moving the first PCO from 0 to $W_{1}$ and moving the second PCO from $\sigma$ to $W_{2}$. Each of the PCOs remains on its own sphere. Since for on-shell three point function, moving the $\mathrm{PCO}$ on the sphere does not have any effect, we expect $\mathbf{B}_{s}$ to vanish in the large $\lambda$ limit. Similar argument can be given for $\mathbf{B}_{t}$. This does not apply to $\mathbf{B}_{u}$ since the initial position of the two PCOs, at 0 and $\sigma$, lie on the same sphere while the final arrangements $W_{1}$ and $W_{2}$ must lie on different spheres. We analyze this as a combination of two moves: first move one of the PCO's from 0 to 1 , and call this contribution $\mathbf{B}_{u}^{\prime}$, and then move the pair of PCO's at 1 and $\sigma$, which are now on different spheres, to $W_{1}$ and $W_{2}$. The latter contribution, called $\mathbf{B}_{u}^{\prime \prime}$ vanishes in the large $\lambda$ limit due to the same arguments as for $\mathbf{B}_{s}$ and $\mathbf{B}_{t}$.

We shall now analyze $\mathbf{B}_{u}^{\prime}$. Due to $\xi-\eta$ charge conservation, only the term proportional to $\eta$ inside the curly bracket contributes. This gives:

$$
\begin{aligned}
\mathbf{B}_{u}^{\prime}= & 2 \frac{\mu^{2}}{2 \pi i} \int_{\partial \mathcal{R}_{u}} d \bar{\sigma}\left\langle c \bar{c} e^{-\phi} \psi^{1} \bar{\partial} X^{2} e^{-i k_{(0)} \cdot X} e^{-i n Y / R}(1) c \bar{c} e^{-\phi} \psi^{1} \bar{\partial} X^{2} e^{i k_{(0)} \cdot X} e^{i n Y / R}(\infty)\right. \\
& \left.\{\xi(0)-\xi(1)\} \eta e^{\phi} \chi \bar{\partial} Y(\sigma) c \bar{c} e^{-\phi} \chi \bar{\partial} Y(0)\right\rangle \\
= & -\frac{\mu^{2}}{16 \pi i} \int_{\partial \mathcal{R}_{u}} d \bar{\sigma} \sigma^{-1}\left(\frac{1}{\bar{\sigma}^{2}}-\frac{n^{2}}{2 R^{2}} \frac{1}{\bar{\sigma}-1}\right)
\end{aligned}
$$

For large $\lambda, \partial \mathcal{R}_{u}$ is a small contour around the origin. In this case the contribution from the term proportional to $(\bar{\sigma}-1)^{-1}$ is suppressed by inverse power of $\lambda$ and can be neglected. The remaining term has the same structure as the right hand side of (7.47) with the roles of $\sigma$ and $\bar{\sigma}$ interchanged. This generates an extra minus sign, leading to (7.48) multiplied by -1 :

$$
\mathbf{B}_{u}^{\prime} \simeq \frac{\mu^{2}}{8} \lambda^{4}\left|h_{3}^{\prime}(1)\right|^{4}
$$


Substituting (7.77), (7.84) and (7.86) into (7.71) we get

$$
-\frac{\mu^{2}}{2}\left\{\tilde{\phi}_{2} \phi_{2} \Psi_{1}^{\mathrm{cl}} \Psi_{1}^{\mathrm{cl}}\right\}=\frac{\mu^{2}}{8} \frac{n^{2}}{R^{2}}-\frac{\mu^{2}}{4} \frac{n^{4}}{R^{4}} \ln \left(\lambda\left|h_{3}^{\prime}(1)\right|\right) .
$$

Using (7.68), (7.87), and the fact that the contribution from the second and third terms on the right hand side of (7.67) vanishes, we get

$$
-\frac{1}{4}\left(k_{(2)}^{2}+n^{2} R^{-2}\right)=-\left(\frac{\mu}{4}-\frac{\mu^{2}}{8}\right) n^{2} R^{-2} .
$$

This gives

$$
k_{(2)}^{2}=-n^{2} R^{-2}\left(1-\mu+\frac{\mu^{2}}{2}\right) .
$$

Although the $\mu$ dependent corrections have the same form as (7.59), there is a subtle difference in the analysis. In the case of bosonic string theory the potential short distance divergence, reflected in the term $I_{1}$ in (7.48) that grows as $\lambda^{4}$ in the large $\lambda$ limit, can be identified to the contribution from the intermediate tachyon state. The naive divergence in the world-sheet integral over $\sigma$ can be traced to the wrong treatment of the tachyon propagator as in (1.2). In contrast the potential short distance divergence in the heterotic string theory, reflected in the term proportional to $\lambda^{4}$ in (7.77), can be traced to the wrong assignment of PCO locations, and is cancelled by the boundary term $\mathbf{B}_{u}^{\prime}$ that corrects the PCO location via vertical integration.

\section{Comments of higher genus amplitudes}

As we have emphasized earlier, there is a subtle difference between the analysis in section 4, 5, 6 and that in section 7. The analysis in sections 4, 5 and 6 was carried out in a manner that is manifestly independent of the string field theory data - choice of local coordinate system and PCO locations. On the other hand the analysis in section 7 required, in the intermediate steps, use of the local coordinate system, e.g. the function $h_{3}(z)$ in (7.41) and $g_{4}(z)$ in (7.15), although at the end the dependence cancelled. There is an intrinsic reason for this difference. The on-shell amplitudes discussed in section 4, 5, 6 are expected to be genuinely independent of the choice of string field theory data, and so it is not surprising that the analysis can be made independent of these choices. The result of section 7 , describing the effect of marginal deformation, is not expected to be manifestly independent of the string field theory data although the dependence on these data is expected to be removable by a redefinition of the deformation parameter $\mu$. This is due to the fact that a change in the string field theory data causes a redefinition of the string fields including the one corresponding to the marginal operator, and such field redefinitions will induce a redefinition of the deformation parameter. Therefore the total independence of the final results (7.59), (7.89) on the string field theory data is accidental, and we expect that in general there will be such dependence of the result on these data.

It is natural to ask if the analysis can be generalized to give a systematic procedure for computing higher genus amplitudes that gives manifestly finite results, and yet minimizes 
the dependence on the choice of string field theory data. To this end note that for higher genus amplitudes, the dependence on the string field theory data of the kind presented in section 7 will always be present even in the absence of marginal deformations. This is due to the fact that at higher genus, under quantum corrections there will be mass and wavefunction renormalizations of all the external states. Now since different choices of string field theory data lead to string field theories that are related by field redefinition, there will be two effects [35, 47, 48]. First the amplitudes computed in different string field theories will differ due to different wave-function renormalizations of external states. Second the definition of the moduli fields, e.g. the string coupling constant encoded in the dilaton, will differ in different string field theories, causing a change in the amplitude. These will lead to ambiguities in the final result that depend on the string field theory data. However such ambiguities can be absorbed into a finite renormalization of external states and the values of the moduli. Therefore, as in this paper, one could proceed with the computation assuming the existence of a consistent set of string field theory data without making a particular choice and then verify at the end that the final result depends on these data only through the normalization of the external states and definition of the moduli fields.

We shall end this section by describing the different kinds of degenerations that we need to deal with for higher genus amplitudes:

1. The first type of degeneration is separating type degeneration with generic momentum flowing across the degenerating punctures. These correspond to the original Riemann surface degenerating into a pair of Riemann surfaces, each of which carries two or more external punctures. These can be treated in the same way as in section 5, 6 and do not introduce any ambiguity in the final result.

2. The second type of degenerations, analyzed extensively in [48], involve separating type degenerations with special momentum flowing across the degenerating punctures. Examples of these involve degenerations where the original Riemann surface degenerates into a pair of Riemann surfaces, one of which carries either no puncture or one puncture. The first one represents tadpole type diagrams with zero momentum flowing across the degenerating puncture while the second one represents mass and wave-function renormalization diagrams with on-shell momentum flowing across the degenerating puncture. Near these degenerations the expansion of the integrand in powers of the variables $u, \bar{u}$ that vanish at the degeneration involves integer exponents and we cannot apply the general trick of section 5, 6 to remove these divergences. There may be genuine divergences of the form $\int d^{2} u /|u|^{2}$ signaling the presence of massless tadpoles and renormalization of physical masses. There may also be ambiguities in determining the $\mathcal{I}^{(k)}$ 's of the form $d u / u$ encoding redefinitions of massless moduli fields and/or external states. We need to use the analog of the procedure described in section 7 to address these cases. The general procedure based on string field theory can be found in [35]. In simple cases, one can follow the procedure described in section 7 to minimize the dependence on the explicit knowledge of string field theory, reproducing the results in [49-51]. 
3. The final category of degenerations involves non-separating type degenerations degenerations where one pinches the handle of a Riemann surface but the Riemann surface still remains connected after degeneration. This case lies in between the two cases described earlier, in that the momentum flowing across the degenerating puncture is a loop momentum that needs to be integrated. For most of the range of integration over momenta the momentum is generic, but on codimension one subspaces of the loop momentum space the $L_{0}+\bar{L}_{0}$ eigenvalue corresponding to that momentum may vanish, causing the integrand to diverge. In four or less dimensions the momentum integrals themselves are divergent reflecting the presence of infrared divergences in the theory and the procedure for getting finite result is complicated - requiring the same methods that are normally used in quantum field theories with massless fields. However in higher than four dimensions there is no genuine divergence and one should be able to extract finite results. Nevertheless the answer is not free from ambiguity without additional input, since the $i \epsilon$ prescription in the integration over the loop momenta is hidden in the prescription of how we deal with the pole in the $1 /\left(L_{0}+\bar{L}_{0}\right)$ factor. This can be done following the procedure described in [23], where we replace the $\left(L_{0}+\bar{L}_{0}\right)^{-1}$ factor by the representation (1.4). This means that if the original integral has the form

$$
\int d q \wedge d \bar{q}|q|^{-2} f(q, \bar{q})=-2 i \int d s \wedge d \theta f(q, \bar{q}), \quad q \equiv e^{-(s+i \theta)},
$$

where we have suppressed the integration over the other moduli, we replace it by ${ }^{12}$

$$
-2 i \int_{0}^{2 \pi} d \theta \int_{s=0}^{\Lambda} d s f(q, \bar{q})-2 i \int_{0}^{2 \pi} d \theta \int_{s=\Lambda}^{\Lambda+i \infty} d s e^{i \epsilon s} f(q, \bar{q}) .
$$

The relation between the variables $q, \bar{q}$ and some predetermined moduli parameters on the punctured Riemann surface can be determined if we know the string field theory data. However this is not necessary. Let us suppose that $u, \bar{u}$ correspond to some other set of variables with the property that $u$ vanishes linearly with $q$ near the degeneration. Parametrizing $u$ as $e^{-t-i \phi}$, we can conclude that if the original integrand has the form

$$
d u \wedge d \bar{u}|u|^{-2} F(u, \bar{u})=-2 i d t \wedge d \phi F(u, \bar{u}),
$$

then we can restrict the range of $u$ integration to $|u| \geq e^{-\Lambda}$ and add to it a term

$$
-2 i \int_{t=\Lambda}^{\Lambda+i \infty} d t \int_{0}^{2 \pi} d \phi e^{i \epsilon t} F(u, \bar{u})
$$

The equivalence between these two procedures - one in $q, \bar{q}$ variable and the other in the $u, \bar{u}$ variable - follows from the fact that near the degeneration $q$ and $u$ are related to each other in a one to one fashion. Therefore if we map the integration contour

\footnotetext{
${ }^{12}$ Even though the Cutkosky rules are not manifest in the procedure described in [23], this has been shown to be equivalent to the procedure of [52] and therefore satisfies the Cutkosky rules [53].
} 


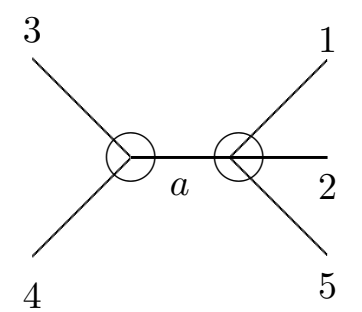

(i)

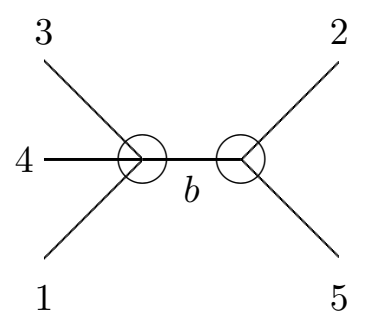

(ii)

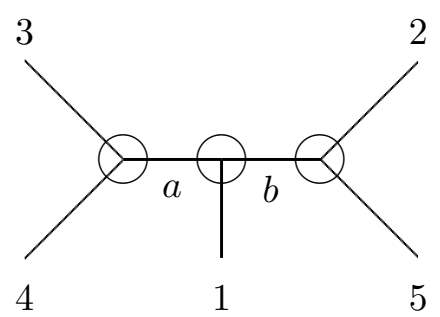

(iii)

Figure 2. Examples of boundaries $\mathcal{C}_{s_{1} \cdots s_{k}}^{(k)}$. The left figure describes $\mathcal{C}_{a}^{(1)}$, the middle figure described $\mathcal{C}_{b}^{(1)}$ and the right figure describes $\mathcal{C}_{a b}^{(2)}$.

used in (8.4) to the $(s, \theta)$ plane, the resulting contour can be smoothly deformed to the contour in (8.2) without passing through any singularity. In dimensions larger than four, and for generic momenta of external states, the integrand $F(u, \bar{u})$ has power law suppression in inverse powers of $-\ln |u|$ that makes the integral convergent from the large $\operatorname{Im}(t)$ region even without the $e^{i \epsilon t}$ factor. Therefore we can compute the integral directly, without having to take limits of integrals. This remains true even for multiple integrals of this kind where more than one handle degenerates simultaneously. An explicit example of this for one loop amplitude can be found in [54] (see e.g. eq. (3.16)).

\section{Acknowledgments}

I wish to thank Roji Pius and Barton Zwiebach for useful discussions and Barton Zwiebach for his very useful comments on an earlier version of this manuscript. This work was supported in part by the J. C. Bose fellowship of the Department of Science and Technology, India and also by the Infosys Chair Professorship.

\section{A Choice of local coordinates on a five punctured sphere}

In this appendix we shall describe, for the five punctured sphere, the relation between the global coordinates $\left(\sigma_{1}, \sigma_{2}\right)$ and the coordinates $u_{s_{1}}, \cdots, u_{s_{k}}, m_{\left(s_{1}, \cdots, s_{k}\right)}$ for some choices of $\left\{s_{1}, \cdots s_{k}\right\}$.

We begin by making a specific choice of global coordinates. This will be done by fixing the puncture locations $y_{1}, \cdots y_{5}$ in the complex plane to be at

$$
y_{1}=\sigma_{1}, \quad y_{2}=\sigma_{2}, \quad y_{3}=1, \quad y_{4}=2, \quad y_{5}=0 .
$$

The boundaries we shall consider are shown in figure 2. These are similar to the Feynman diagrams shown in figure 1, but figure 2 should be regarded as depicting regions near those represented by the Feynman diagrams in figure 1 for $|q| \sim e^{-\Lambda}$ not necessarily the precise regions that follow from a string field theory. For example figure 2(i) represents region near the boundary $\mathcal{C}_{a}^{(1)}$ where the original sphere is near a degeneration into a four punctured sphere carrying the original punctures 1,2 and 5 and a three punctured sphere carrying 
the original punctures 3 and 4 . Our goal will be to introduce the coordinates $u_{a}, m_{(a)}$ near this boundary in terms of the global coordinates $\sigma_{1}, \sigma_{2}$. This is done as follows. Let us take the three punctured sphere on the left, carrying global coordinate $z$, to have puncture 3 at $z=1$, puncture 4 at $z=2$ and the sewing puncture at $z=0$. We also take the four punctured sphere on the right, carrying global coordinate $z^{\prime}$, to have puncture 1 at $z^{\prime}=m_{(a)}$, puncture 2 at $z^{\prime}=2$, puncture 5 at $z^{\prime}=1$ and the sewing puncture at $z^{\prime}=0$. $m_{(a)}$ should keep a finite distance away from 0,1 and 2 so that the four punctured sphere is not close to degeneration - as will be discussed later, for $m_{(a)}$ close to 0,1 or 2 , we need to choose the coordinate systems differently. We now sew the two spheres via the relation

$$
z z^{\prime}=\hat{u}_{a}
$$

We have used $\hat{u}_{a}$ instead of $u_{a}$ to take into account the fact that the correct candidate for the coordinate $u_{a}$ may have different form in different domains in the moduli space. We shall see that while the $\hat{u}_{a}$ appearing in the above equation is the correct choice of $u_{a}$ as long as $m_{(a)}$ is kept away from 0,1 and 2, we need modifications when $m_{(a)}$ approaches any of these points. In the $z$ coordinate the punctures are located at:

$$
3: z=1, \quad 4: z=2, \quad 5: z=\hat{u}_{a}, \quad 1: z=\hat{u}_{a} / m_{(a)}, \quad 2: z=\hat{u}_{a} / 2 .
$$

In order to bring the 5 th puncture at 0 leaving the third and fourth punctures at 1 and 2 respectively, we make a change of coordinates:

$$
y=\frac{2\left(z-\hat{u}_{a}\right)}{\hat{u}_{a}(z-3)+2} .
$$

The location of the punctures 1 and 2 in the $y$ plane are now given by, respectively,

$$
\sigma_{1}=\frac{2\left(1-m_{(a)}\right) \hat{u}_{a}}{\hat{u}_{a}\left(\hat{u}_{a}-3 m_{(a)}\right)+2 m_{(a)}}, \quad \sigma_{2}=-\frac{2 \hat{u}_{a}}{\hat{u}_{a}\left(\hat{u}_{a}-6\right)+4} .
$$

Since we shall use this formula only for small $\hat{u}_{a}$, we shall replace (A.5) by a simpler equation:

$$
\sigma_{1}=\frac{\left(1-m_{(a)}\right) \hat{u}_{a}}{m_{(a)}}, \quad \sigma_{2}=-\frac{\hat{u}_{a}}{2} .
$$

We shall take (A.6) as the definitions of the coordinates $\left\{\hat{u}_{a}, m_{(a)}\right\}$ in terms of the global coordinates $\sigma_{1}, \sigma_{2}$ of the moduli space near $\mathcal{C}_{a}^{(1)}$. These can be taken to represent the coordinates $\left(u_{a}, m_{(a)}\right)$ in the notation of section 5 when $m_{(a)}$ is not close to 0,1 or 2 .

Let us now turn to the region near the boundary $\mathcal{C}_{b}^{(1)}$ represented by figure 2(ii). We take the four punctured sphere on the left, carrying global coordinate $z$, to have puncture 3 at $z=1$, puncture 4 at $z=2$, puncture 1 at $z=m_{(b)}$ and the sewing puncture at $z=0$. $m_{(b)}$ needs to keep finite distance away from 0,1 and 2 so that this sphere is not close to degeneration. We also take the three punctured sphere on the right, carrying global coordinate $z^{\prime}$, to have puncture 2 at $z^{\prime}=2$, puncture 5 at $z^{\prime}=1$ and the sewing puncture at $z^{\prime}=0$. We now sew the two Riemann surfaces via the relation

$$
z z^{\prime}=\hat{u}_{b}
$$


In the $z$ coordinate the punctures are located at:

$$
3: z=1, \quad 4: z=2, \quad 5: z=\hat{u}_{b}, \quad 1: z=m_{(b)}, \quad 2: z=\hat{u}_{b} / 2 .
$$

In order to bring the 5th puncture to 0 leaving the third and fourth punctures at 1 and 2 respectively, we make a change of coordinates:

$$
y=\frac{2\left(z-\hat{u}_{b}\right)}{\hat{u}_{b}(z-3)+2} .
$$

The location of the punctures 1 and 2 in the $y$ plane are now given by, respectively,

$$
\sigma_{1}=\frac{2\left(m_{(b)}-\hat{u}_{b}\right)}{\hat{u}_{b}\left(m_{(b)}-3\right)+2}, \quad \sigma_{2}=-\frac{2 \hat{u}_{b}}{\hat{u}_{b}\left(\hat{u}_{b}-6\right)+4} .
$$

Again, since we shall use this coordinate system for small $\hat{u}_{b}$, we replace this by a simpler set of equations:

$$
\sigma_{1}=m_{(b)}, \quad \sigma_{2}=-\frac{\hat{u}_{b}}{2} .
$$

(A.11) defines the coordinates $\hat{u}_{b}, m_{(b)}$ appropriate near $\mathcal{C}_{(b)}$ in terms of the global coordinates $\sigma_{1}, \sigma_{2}$ of the moduli space. These can be taken to represent the coordinates $\left(u_{b}, m_{(b)}\right)$ in the notation of section 5 when $m_{(b)}$ is not close to 0,1 or 2 .

Next we turn to the region near $\mathcal{C}_{a b}^{(2)}$ represented by the diagram 2(iii). Keeping in mind that the variables $\hat{u}_{a}$ and $\hat{u}_{b}$ introduced earlier may not exactly match with the variables suitable for parametrizing the region near $\mathcal{C}_{a b}^{(2)}$, we shall denote the new parameters by $\tilde{u}_{a}$ and $\tilde{u}_{b}$. We take the left sphere carrying global coordinate $z$ to have puncture 3 at $z=1$, puncture 4 at $z=2$ and the sewing puncture at $z=0$, the middle sphere carrying global coordinate $z^{\prime}$ to have puncture 1 at $z^{\prime}=1$, the left sewing puncture at $z^{\prime}=0$ and the right sewing puncture at $z^{\prime}=\infty$ and the right sphere carrying global coordinate $z^{\prime \prime}$ to have the sewing puncture at $z^{\prime \prime}=0$, puncture 2 at $z^{\prime \prime}=2$ and puncture 5 at $z^{\prime \prime}=1$. We now sew the three spheres via the relation

$$
z z^{\prime}=\tilde{u}_{a}, \quad z^{\prime \prime} / z^{\prime}=\tilde{u}_{b} .
$$

In the $z$ coordinate the punctures are located at:

$$
3: z=1, \quad 4: z=2, \quad 5: z=\tilde{u}_{a} \tilde{u}_{b}, \quad 1: z=\tilde{u}_{a}, \quad 2: z=\tilde{u}_{a} \tilde{u}_{b} / 2 .
$$

We now introduce new coordinate

$$
y=\frac{2\left(z-\tilde{u}_{a} \tilde{u}_{b}\right)}{\tilde{u}_{a} \tilde{u}_{b}(z-3)+2},
$$

so that we have

$$
y_{3}=1, \quad y_{4}=2, \quad y_{5}=0 .
$$

The locations of the punctures 1 and 2 in the $y$ plane are now given by, respectively,

$$
\sigma_{1}=\frac{2\left(1-\tilde{u}_{b}\right) \tilde{u}_{a}}{\tilde{u}_{a} \tilde{u}_{b}\left(\tilde{u}_{a}-3\right)+2}, \quad \sigma_{2}=-\frac{2 \tilde{u}_{a} \tilde{u}_{b}}{\tilde{u}_{a} \tilde{u}_{b}\left(\tilde{u}_{a} \tilde{u}_{b}-6\right)+4} .
$$


Again since we shall be using this change of coordinates for small $\tilde{u}_{a}, \tilde{u}_{b}$, we shall replace this by

$$
\sigma_{1}=\tilde{u}_{a}, \quad \sigma_{2}=-\frac{\tilde{u}_{a} \tilde{u}_{b}}{2}
$$

(A.17) gives the definition of the coordinates $\tilde{u}_{a}, \tilde{u}_{b}$ appropriate near $\mathcal{C}_{(a b)}^{(2)}$ in terms of the global coordinates $\sigma_{1}, \sigma_{2}$ of the moduli space. Comparing (A.6), (A.11) and (A.17) we can find the relations between the coordinate systems near $\mathcal{C}_{a}^{(1)}, \mathcal{C}_{a b}^{(2)}$ and $\mathcal{C}_{b}^{(1)}$ :

$$
\hat{u}_{a}=\tilde{u}_{a} \tilde{u}_{b}, \quad m_{(a)}=\frac{\tilde{u}_{b}}{1+\tilde{u}_{b}}, \quad \hat{u}_{b}=\tilde{u}_{a} \tilde{u}_{b}, \quad m_{(b)}=\tilde{u}_{a} .
$$

We now recall that for figure 2(i), identification of the coordinate system $\left(\hat{u}_{a}, m_{(a)}\right)$ with the coordinates $\left(u_{a}, m_{(a)}\right)$ introduced in section 5 breaks down for $m_{(a)}$ close to 0 since the right sphere degenerates in this limit. By examining the choice of coordinates of the punctures on the original sphere one can see that this degeneration is precisely the one depicted in figure 2(iii). Therefore in this region we can identify $\left(\tilde{u}_{a}, m_{(a)}\right)$, instead of $\left(\hat{u}_{a}, m_{(a)}\right)$, with the coordinates $\left(u_{a}, m_{(a)}\right)$ introduced in section 5. Similar modifications must also be made when $m_{(a)}$ approaches 1 and 2 by analyzing good coordinate systems near other degenerations. The choice of the coordinate system $\left(u_{b}, m_{(b)}\right)$ needs to be similarly modified when $m_{(b)}$ approaches 0,1 and 2. For example when $m_{(b)}$ approaches 0 we can use $\left(\tilde{u}_{b}, m_{(b)}\right)$ to label coordinates near $\mathcal{C}_{(b)}^{(1)}$.

Using this coordinate system we can also define the boundaries $\mathcal{C}_{a}^{(1)}$ and $\mathcal{C}_{b}^{(1)}$. For example when $m_{(a)}$ is finite distance away from 0,1 and 2 , we can use $\left|\hat{u}_{a}\right|=\epsilon$ for defining $\mathcal{C}_{a}^{(1)}$, but when $m_{(a)}$ is close to 0 , we use $\left|\tilde{u}_{a}\right|=\epsilon$ as the definition of $\mathcal{C}_{a}^{(1)}$. Similarly we can define $\mathcal{C}_{b}^{(1)}$ to be given by $\left|\hat{u}_{b}\right|=\epsilon$ when $m_{(b)}$ is away from 0,1 and 2 but $\left|\tilde{u}_{b}\right|=\epsilon$ when $m_{(b)}$ is close to zero. These can be formally stated as follows. Let $H(x)$ be a smooth function of a complex variable $x$ that approaches 1 for large $|x|$ and zero for small $|x|$, e.g.

$$
H(x) \equiv \frac{|x|^{2}}{|x|^{2}+\eta^{2}}
$$

where $\eta$ is a fixed number. Then we define:

$\mathcal{C}_{a}^{(1)}:\left|\hat{u}_{a}\right|=\epsilon H\left(m_{(a)}\right)+\epsilon\left|m_{(a)}\right|\left(1-H\left(m_{(a)}\right)\right), \quad \mathcal{C}_{b}^{(1)}:\left|\hat{u}_{b}\right|=\epsilon H\left(m_{(b)}\right)+\epsilon\left|m_{(b)}\right|\left(1-H\left(m_{(b)}\right)\right)$.

When $m_{(a)}\left(m_{(b)}\right)$ is close to 1 or 2 , the definitions of $\mathcal{C}_{a}^{(1)}\left(\mathcal{C}_{b}^{(2)}\right)$ need to be further modified along the same line. $\mathcal{C}_{a b}^{(1)}$ is simply the intersection of these two subspaces, given approximately by $\left|\tilde{u}_{a}\right| \simeq\left|\tilde{u}_{b}\right| \simeq \epsilon$ for small $\epsilon$. Note however that we do not need to take the $\epsilon \rightarrow 0$ limit since (5.8) gives the correct result even when the $\mathcal{C}_{s}^{(1)}$, s have finite size.

Given the original integrand $\mathcal{I}^{(0)}$ for the five point function, computed from correlation functions of vertex operators in the conformal field theory, we can now construct the differential forms $\mathcal{I}_{a}^{(1)}, \mathcal{I}_{b}^{(1)}$ and $\mathcal{I}_{a b}^{(2)}$ as follows. We first consider expansions of $\mathcal{I}^{(0)}$ near 
$\mathcal{C}_{a}^{(1)}$ and $\mathcal{C}_{b}^{(1)}$

$$
\begin{aligned}
& \mathcal{I}^{(0)}=d \hat{u}_{a} \wedge d \overline{\hat{u}}_{a} \wedge d m_{(a)} \wedge d \bar{m}_{(a)} \sum_{i} C_{i}\left(m_{(a)}\right) \hat{u}_{a}^{-1+\alpha_{i}} \overline{\hat{u}}_{a}^{-1+\beta_{i}} \quad \text { near } \mathcal{C}_{a}^{(1)} \\
& =d \hat{u}_{b} \wedge d \overline{\hat{u}}_{b} \wedge d m_{(b)} \wedge d \bar{m}_{(b)} \sum_{i} \widetilde{C}_{i}\left(m_{(b)}\right) \hat{u}_{b}^{-1+\tilde{\alpha}_{i}} \overline{\hat{u}}_{b}^{-1+\tilde{\beta}_{i}} \quad \text { near } \mathcal{C}_{b}^{(1)} .
\end{aligned}
$$

We now obtain $\mathcal{I}_{a}^{(1)}$ by solving the $d \mathcal{I}_{a}^{(1)}=\mathcal{I}^{(0)}$ near $\mathcal{C}_{a}^{(1)}$. A solution is

$$
\begin{aligned}
\mathcal{I}_{a}^{(1)} & =-d \hat{u}_{a} \wedge d m_{(a)} \wedge d \bar{m}_{(a)} \sum_{i}\left(\beta_{i}\right)^{-1} C_{i}\left(m_{(a)}\right) \hat{u}_{a}^{-1+\alpha_{i}} \overline{\hat{u}}_{a}^{\beta_{i}} \\
& =-d \tilde{u}_{a} \wedge d \tilde{u}_{b} \wedge d \overline{\tilde{u}}_{b}\left|1+\tilde{u}_{b}\right|^{-4} \tilde{u}_{b} \sum_{i}\left(\beta_{i}\right)^{-1} C_{i}\left(\frac{\tilde{u}_{b}}{1+\tilde{u}_{b}}\right)\left(\tilde{u}_{a} \tilde{u}_{b}\right)^{-1+\alpha_{i}}\left(\overline{\tilde{u}}_{a} \overline{\tilde{u}}_{b}\right)^{\beta_{i}}
\end{aligned}
$$

where in the second line we have displayed its behavior in the coordinate system appropriate near $\mathcal{C}_{a b}^{(2)}$ using the coordinate transformations (A.18). Similarly we have

$$
\begin{aligned}
\mathcal{I}_{b}^{(1)} & =-d \hat{u}_{b} \wedge d m_{(b)} \wedge d \bar{m}_{(b)} \sum_{i}\left(\tilde{\beta}_{i}\right)^{-1} \widetilde{C}_{i}\left(m_{(b)}\right) \hat{u}_{b}^{-1+\tilde{\alpha}_{i}} \overline{\hat{u}}_{b}^{\tilde{\beta}_{i}} \\
& =-d \tilde{u}_{b} \wedge d \tilde{u}_{a} \wedge d \overline{\tilde{u}}_{a} \tilde{u}_{a} \sum_{i}\left(\tilde{\beta}_{i}\right)^{-1} \widetilde{C}_{i}\left(\tilde{u}_{a}\right)\left(\tilde{u}_{a} \tilde{u}_{b}\right)^{-1+\tilde{\alpha}_{i}}\left(\overline{\tilde{u}}_{a} \overline{\tilde{u}}_{b}\right)^{\tilde{\beta}_{i}}
\end{aligned}
$$

We can now expand $C_{i}$ and $\widetilde{C}_{i}$ in the second lines of (A.22) and (A.23) in power series expansion in $\tilde{u}_{b}$ and $\tilde{u}_{a}$ respectively to find the expressions for $\mathcal{I}_{a}^{(1)}$ and $\mathcal{I}_{b}^{(1)}$ near $\mathcal{C}_{a b}^{(2)} \cdot \mathcal{I}_{a b}^{(2)}$ is then obtained by solving the equation:

$$
d \mathcal{I}_{a b}^{(2)}=\mathcal{I}_{a}^{(1)}-\mathcal{I}_{b}^{(1)}
$$

\section{B Analysis of $\mathrm{B}_{u}^{\prime \prime}, \mathrm{B}_{s}$ and $\mathrm{B}_{t}$}

In this appendix we shall show that $\mathbf{B}_{s}, \mathbf{B}_{t}$ and $\mathbf{B}_{u}^{\prime \prime}$, defined in (7.80), (7.83), vanish in the large $\lambda$ limit. We begin with $\mathbf{B}_{s}$. Keeping only the part of the correlator in (7.80) that does not vanish by ghost charge conservation, we have

$$
\begin{aligned}
& \mathbf{B}_{s}=-2 \frac{\mu^{2}}{2 \pi i} \int_{\partial \mathcal{R}_{s}} d \bar{\sigma}\left\langle c \bar{c} e^{-\phi} \psi^{1} \bar{\partial} X^{2} e^{-i k_{(0)} \cdot X} e^{-i n Y / R}(1) c \bar{c} e^{-\phi} \psi^{1} \bar{\partial} X^{2} e^{i k_{(0)} \cdot X} e^{i n Y / R}(\infty)\right. \\
& \quad\left[-\left\{\xi(0)-\xi\left(W_{1}\right)\right\} \eta e^{\phi} \chi(\sigma) \bar{\partial} Y(\sigma) c \bar{c} e^{-\phi} \chi \bar{\partial} Y(0)\right. \\
& \left.\left.\quad-\left\{\xi(\sigma)-\xi\left(W_{2}\right)\right\}\left\{\partial \eta e^{2 \phi} b\left(W_{1}\right)+\partial\left(\eta e^{2 \phi} b\left(W_{1}\right)\right)\right\} c e^{-\phi} \chi \bar{\partial} Y(\sigma) c \bar{c} e^{-\phi} \chi \bar{\partial} Y(0)\right]\right\rangle .
\end{aligned}
$$

For large $\lambda, \partial \mathcal{R}_{s}$ represents a contour around 1 of linear size of order $\lambda^{-2}$. Also $W_{1}$ is a point at finite distance away from 1 and $W_{2}$ is a point within distance $\lambda^{-2}$ of 1 . We shall now estimate the integrand for $\sigma \in \partial \mathcal{R}_{s}$ and show that the integral is suppressed in the large $\lambda$ limit. The integration measure $d \bar{\sigma}$ contributes a factor of $\lambda^{-2}$ since the contour has linear size $\lambda^{-2}$. Therefore the integrand must grow as $\lambda^{2}$ for getting a finite contribution. For the term in the second line of (B.1) the $b, c, \bar{b}, \bar{c}$ correlators give finite contribution, 
the $\phi$ correlator gives a contribution of order $(\sigma-1) \sim \lambda^{-2}$, the $\xi, \eta$ correlator gives finite contribution and the matter correlator gives a contribution of order $(\sigma-1)^{-1} \sim \lambda^{2}$ from the $Y$ contribution. Therefore the correlator grows as order $\lambda^{0}$ and its contribution to the integral vanishes for large $\lambda$. For the term in the third line of (B.1) the $b, c, \bar{b}, \bar{c}$ correlators give a contribution of order $(\sigma-1) \sim \lambda^{-2}$ from the $c$-c operator product, the $\phi$ correlator gives a contribution of order $(\sigma-1)^{-1} \sim \lambda^{2}$, the matter correlator gives a contribution of order $(\sigma-1)^{-1} \sim \lambda^{2}$ from the $Y$ contribution and the $\xi, \eta$ correlator gives a contribution of order $\left(\sigma-W_{2}\right) \sim \lambda^{-2}$ due to the fact that the points $\sigma$ and $W_{2}$ where $\xi$ is inserted are within a distance $\lambda^{-2}$ of each other. This again makes the integrand to be of order $\lambda^{0}$ in the large $\lambda$ limit, making the integral vanish in this limit. This shows that $\mathbf{B}_{s}$ vanishes.

A similar analysis can be carried out for $\mathbf{B}_{t}$ which has the same expression as (B.1) except that the integration contour $\partial \mathcal{R}_{t}$ lies in the large $\sigma$ region $\left(\sigma \sim \lambda^{2}\right), W_{1}$ lies at a finite point and $W_{2}$ also becomes large of order $\lambda^{2}$. However this case is related to that for $\mathbf{B}_{s}$ via a $z \rightarrow z /(z-1)$ transformation accompanied by a reversal in sign of $k_{(0)}$ and $n$. Therefore vanishing of $\mathbf{B}_{s}$ in the large $\lambda$ limit also implies vanishing of $\mathbf{B}_{t}$.

Let us now turn to $\mathbf{B}_{u}^{\prime \prime}$ which, according to (7.83), is given by

$$
\begin{aligned}
\mathbf{B}_{u}^{\prime \prime}= & -2 \frac{\mu^{2}}{2 \pi i} \int_{\partial \mathcal{R}_{u}} d \bar{\sigma}\left\langle c \bar{c} e^{-\phi} \psi^{1} \bar{\partial} X^{2} e^{-i k_{(0)} \cdot X} e^{-i n Y / R}(1) c \bar{c} e^{-\phi} \psi^{1} \bar{\partial} X^{2} e^{i k_{(0)} \cdot X} e^{i n Y / R}(\infty)\right. \\
& {\left[-\left\{\xi(1)-\xi\left(W_{1}\right)\right\} \eta e^{\phi} \chi(\sigma) \bar{\partial} Y(\sigma) c \bar{c} e^{-\phi} \chi \bar{\partial} Y(0)\right.} \\
& \left.\left.-\left\{\xi(\sigma)-\xi\left(W_{2}\right)\right\}\left\{\partial \eta e^{2 \phi} b\left(W_{1}\right)+\partial\left(\eta e^{2 \phi} b\left(W_{1}\right)\right)\right\} c e^{-\phi} \chi \bar{\partial} Y(\sigma) c \bar{c} e^{-\phi} \chi \bar{\partial} Y(0)\right]\right\rangle .
\end{aligned}
$$

In this case the integration contour $\partial \mathcal{R}_{u}$ encloses 0 and, according to (3.13), (3.17), represents approximately a circle of radius $\propto \lambda^{-2}$ around the origin, with corrections of order $\lambda^{-4}$. The point $W_{1}$ is at finite distance away from 0 and the point $W_{2}$ is within a distance of order $\lambda^{-2}$ of 0 . Now it follows from the analysis in section 3.1, 3.2 that $W_{1}$ and $W_{2}$ are holomorphic functions of $q$ and hence of $\sigma$. Evaluating the correlator in (B.2) we find that $\mathbf{B}_{u}^{\prime \prime}$ has the form:

$$
\mathbf{B}_{u}^{\prime \prime}=\int_{\partial \mathcal{R}_{u}} d \bar{\sigma} f(\sigma)\left(\frac{1}{\bar{\sigma}^{2}}-\frac{n^{2}}{2 R^{2}} \frac{1}{\bar{\sigma}-1}\right)
$$

for some holomorphic function $f(\sigma)$ with a regular Taylor series expansion around $\sigma=0$. It follows from this that for large $\lambda, \mathbf{B}_{u}^{\prime \prime}$ is suppressed by inverse powers of $\lambda$.

Open Access. This article is distributed under the terms of the Creative Commons Attribution License (CC-BY 4.0), which permits any use, distribution and reproduction in any medium, provided the original author(s) and source are credited.

\section{References}

[1] M. Schnabl, Analytic solution for tachyon condensation in open string field theory, Adv. Theor. Math. Phys. 10 (2006) 433 [hep-th/0511286] [InSPIRE].

[2] C. de Lacroix, H. Erbin, S.P. Kashyap, A. Sen and M. Verma, Closed Superstring Field Theory and its Applications, Int. J. Mod. Phys. A 32 (2017) 1730021 [arXiv:1703.06410] [INSPIRE]. 
[3] D. Friedan, E.J. Martinec and S.H. Shenker, Conformal Invariance, Supersymmetry and String Theory, Nucl. Phys. B 271 (1986) 93 [INSPIRE].

[4] E. Witten, Noncommutative Geometry and String Field Theory, Nucl. Phys. B 268 (1986) 253 [INSPIRE].

[5] M. Saadi and B. Zwiebach, Closed String Field Theory from Polyhedra, Annals Phys. 192 (1989) 213 [INSPIRE].

[6] T. Kugo, H. Kunitomo and K. Suehiro, Nonpolynomial Closed String Field Theory, Phys. Lett. B 226 (1989) 48 [INSPIRE].

[7] T. Kugo and K. Suehiro, Nonpolynomial Closed String Field Theory: Action and Its Gauge Invariance, Nucl. Phys. B 337 (1990) 434 [inSPIRE].

[8] H. Sonoda and B. Zwiebach, Closed String Field Theory Loops With Symmetric Factorizable Quadratic Differentials, Nucl. Phys. B 331 (1990) 592 [INSPIRE].

[9] B. Zwiebach, Closed string field theory: Quantum action and the B-V master equation, Nucl. Phys. B 390 (1993) 33 [hep-th/9206084] [INSPIRE].

[10] B. Zwiebach, Oriented open-closed string theory revisited, Annals Phys. 267 (1998) 193 [hep-th/9705241] [INSPIRE].

[11] R. Saroja and A. Sen, Picture changing operators in closed fermionic string field theory, Phys. Lett. B 286 (1992) 256 [hep-th/9202087] [INSPIRE].

[12] T. Erler, S. Konopka and I. Sachs, Resolving Witten's superstring field theory, JHEP 04 (2014) 150 [arXiv:1312.2948] [INSPIRE].

[13] T. Erler, S. Konopka and I. Sachs, NS-NS Sector of Closed Superstring Field Theory, JHEP 08 (2014) 158 [arXiv: 1403.0940] [INSPIRE].

[14] A. Sen, BV Master Action for Heterotic and Type II String Field Theories, JHEP 02 (2016) 087 [arXiv: 1508.05387] [INSPIRE].

[15] T. Erler, Y. Okawa and T. Takezaki, Complete Action for Open Superstring Field Theory with Cyclic $A_{\infty}$ Structure, JHEP 08 (2016) 012 [arXiv: 1602.02582] [INSPIRE].

[16] S. Konopka and I. Sachs, Open Superstring Field Theory on the Restricted Hilbert Space, JHEP 04 (2016) 164 [arXiv: 1602.02583] [INSPIRE].

[17] N. Berkovits, SuperPoincaré invariant superstring field theory, Nucl. Phys. B 450 (1995) 90 [Erratum ibid. B 459 (1996) 439] [hep-th/9503099] [INSPIRE].

[18] N. Berkovits, The Ramond sector of open superstring field theory, JHEP 11 (2001) 047 [hep-th/0109100] [INSPIRE].

[19] Y. Okawa and B. Zwiebach, Heterotic string field theory, JHEP 07 (2004) 042 [hep-th/0406212] [INSPIRE].

[20] N. Berkovits, Y. Okawa and B. Zwiebach, WZW-like action for heterotic string field theory, JHEP 11 (2004) 038 [hep-th/0409018] [INSPIRE].

[21] H. Kunitomo and Y. Okawa, Complete action for open superstring field theory, PTEP 2016 (2016) 023B01 [arXiv:1508.00366] [INSPIRE].

[22] A.J. Hanson and J.-P. Sha, A contour integral representation for the dual five-point function and a symmetry of the genus-4 surface in $R^{6}$, J. Phys. A 39 (2006) 2509 [math-ph/0510064] [INSPIRE]. 
[23] E. Witten, The Feynman ie in String Theory, JHEP 04 (2015) 055 [arXiv:1307.5124] [INSPIRE].

[24] S. Mizera, Combinatorics and Topology of Kawai-Lewellen-Tye Relations, JHEP 08 (2017) 097 [arXiv: 1706 . 08527] [INSPIRE].

[25] W. Siegel, Covariantly Second Quantized String. 2, Phys. Lett. 149B (1984) 157 [InSPIRE].

[26] H. Hata and B. Zwiebach, Developing the covariant Batalin-Vilkovisky approach to string theory, Annals Phys. 229 (1994) 177 [hep-th/9301097] [INSPIRE].

[27] A. Sen and B. Zwiebach, A proof of local background independence of classical closed string field theory, Nucl. Phys. B 414 (1994) 649 [hep-th/9307088] [INSPIRE].

[28] A. Sen and B. Zwiebach, Quantum background independence of closed string field theory, Nucl. Phys. B 423 (1994) 580 [hep-th/9311009] [InSPIRE].

[29] A. Sen, Background Independence of Closed Superstring Field Theory, JHEP 02 (2018) 155 [arXiv: 1711.08468] [INSPIRE].

[30] M. Schnabl, Comments on marginal deformations in open string field theory, Phys. Lett. B 654 (2007) 194 [hep-th/0701248] [INSPIRE].

[31] M. Kiermaier, Y. Okawa, L. Rastelli and B. Zwiebach, Analytic solutions for marginal deformations in open string field theory, JHEP 01 (2008) 028 [hep-th/0701249] [INSPIRE].

[32] E. Fuchs, M. Kroyter and R. Potting, Marginal deformations in string field theory, JHEP 09 (2007) 101 [arXiv:0704.2222] [INSPIRE].

[33] M. Kiermaier, Y. Okawa and P. Soler, Solutions from boundary condition changing operators in open string field theory, JHEP 03 (2011) 122 [arXiv: 1009.6185] [INSPIRE].

[34] T. Erler and C. Maccaferri, String Field Theory Solution for Any Open String Background, JHEP 10 (2014) 029 [arXiv: 1406.3021] [INSPIRE].

[35] A. Sen, Gauge Invariant 1PI Effective Action for Superstring Field Theory, JHEP 06 (2015) 022 [arXiv: 1411.7478] [INSPIRE].

[36] A. Sen, Supersymmetry Restoration in Superstring Perturbation Theory, JHEP 12 (2015) 075 [arXiv: 1508.02481] [INSPIRE].

[37] S. Mukherji and A. Sen, Some all order classical solutions in nonpolynomial closed string field theory, Nucl. Phys. B 363 (1991) 639 [InSPIRE].

[38] T. Kugo and B. Zwiebach, Target space duality as a symmetry of string field theory, Prog. Theor. Phys. 87 (1992) 801 [hep-th/9201040] [INSPIRE].

[39] M. Cho, S. Collier and X. Yin, Strings in Ramond-Ramond Backgrounds from the Neveu-Schwarz-Ramond Formalism, arXiv:1811.00032 [INSPIRE].

[40] S.F. Moosavian and R. Pius, Hyperbolic Geometry of Superstring Perturbation Theory, arXiv: 1703.10563 [INSPIRE].

[41] S.F. Moosavian and R. Pius, Hyperbolic geometry and closed bosonic string field theory. Part I. The string vertices via hyperbolic Riemann surfaces, JHEP 08 (2019) 157 [arXiv: 1706. 07366] [INSPIRE].

[42] S.F. Moosavian and R. Pius, Hyperbolic geometry and closed bosonic string field theory. Part II. The rules for evaluating the quantum BV master action, JHEP 08 (2019) 177 [arXiv: 1708.04977] [INSPIRE]. 
[43] S. Ghosh and S. Raju, Breakdown of String Perturbation Theory for Many External Particles, Phys. Rev. Lett. 118 (2017) 131602 [arXiv:1611.08003] [INSPIRE].

[44] P. Di Vecchia, R. Nakayama, J.L. Petersen and S. Sciuto, Properties of the Three Reggeon Vertex in String Theories, Nucl. Phys. B 282 (1987) 103 [InSPIRE].

[45] A. Sen, Off-shell Amplitudes in Superstring Theory, Fortsch. Phys. 63 (2015) 149 [arXiv: 1408.0571] [INSPIRE].

[46] A. Sen and E. Witten, Filling the gaps with PCO's, JHEP 09 (2015) 004 [arXiv: 1504.00609] [INSPIRE].

[47] J.J. Atick, G.W. Moore and A. Sen, Catoptric Tadpoles, Nucl. Phys. B 307 (1988) 221 [INSPIRE].

[48] E. Witten, Superstring Perturbation Theory Revisited, arXiv:1209.5461 [INSPIRE].

[49] R. Pius, A. Rudra and A. Sen, Mass Renormalization in String Theory: Special States, JHEP 07 (2014) 058 [arXiv:1311.1257] [INSPIRE].

[50] R. Pius, A. Rudra and A. Sen, Mass Renormalization in String Theory: General States, JHEP 07 (2014) 062 [arXiv: 1401.7014] [inSPIRE].

[51] R. Pius, A. Rudra and A. Sen, String Perturbation Theory Around Dynamically Shifted Vacuum, JHEP 10 (2014) 070 [arXiv: 1404.6254] [INSPIRE].

[52] R. Pius and A. Sen, Cutkosky rules for superstring field theory, JHEP 10 (2016) 024 [Erratum ibid. 09 (2018) 122] [arXiv: 1604.01783] [INSPIRE].

[53] A. Sen, Equivalence of Two Contour Prescriptions in Superstring Perturbation Theory, JHEP 04 (2017) 025 [arXiv: 1610.00443] [INSPIRE].

[54] A. Sen, One Loop Mass Renormalization of Unstable Particles in Superstring Theory, JHEP 11 (2016) 050 [arXiv: 1607.06500] [INSPIRE]. 